\title{
Criticality Accident Alarm System Shielding Benchmark: Integral Experiment Request 498, Critical Engineering Decision 2 Report
}

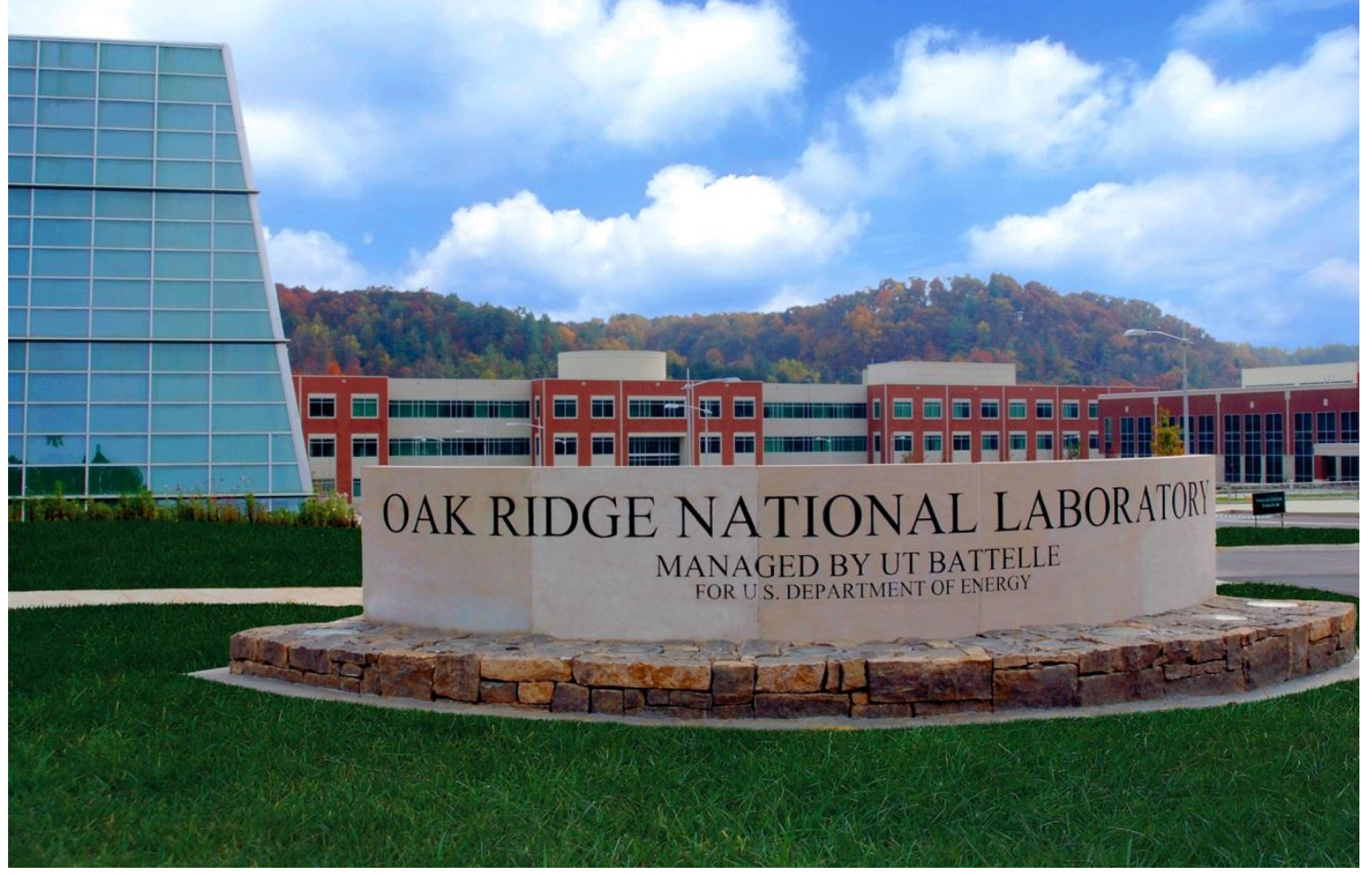

Riley Cumberland

Cihangir Celik Mathieu Dupont

Doug Bowen

September 30, 2021

Approved for public release.

Distribution is unlimited. 


\title{
DOCUMENT AVAILABILITY
}

Reports produced after January 1, 1996, are generally available free via US Department of Energy (DOE) SciTech Connect.

Website www.osti.gov

Reports produced before January 1, 1996, may be purchased by members of the public from the following source:

\author{
National Technical Information Service \\ 5285 Port Royal Road \\ Springfield, VA 22161 \\ Telephone 703-605-6000 (1-800-553-6847) \\ TDD 703-487-4639 \\ Fax 703-605-6900 \\ E-mail info@ntis.gov \\ Website http://classic.ntis.gov/
}

Reports are available to DOE employees, DOE contractors, Energy Technology Data Exchange representatives, and International Nuclear Information System representatives from the following source:

Office of Scientific and Technical Information

PO Box 62

Oak Ridge, TN 37831

Telephone 865-576-8401

Fax 865-576-5728

E-mail reports@osti.gov

Website http://www.osti.gov/

This report was prepared as an account of work sponsored by an agency of the United States Government. Neither the United States Government nor any agency thereof, nor any of their employees, makes any warranty, express or implied, or assumes any legal liability or responsibility for the accuracy, completeness, or usefulness of any information, apparatus, product, or process disclosed, or represents that its use would not infringe privately owned rights. Reference herein to any specific commercial product, process, or service by trade name, trademark, manufacturer, or otherwise, does not necessarily constitute or imply its endorsement, recommendation, or favoring by the United States Government or any agency thereof. The views and opinions of authors expressed herein do not necessarily state or reflect those of the United States Government or any agency thereof. 
Nuclear Energy and Fuel Cycle Division

\title{
CRITICALITY ACCIDENT ALARM SYSTEM SHIELDING BENCHMARK: INTEGRAL EXPERIMENT REQUEST 498, CRITICAL ENGINEERING DECISION 2 REPORT
}

\author{
Riley Cumberland \\ Cihangir Celik \\ Mathieu Dupont \\ Doug Bowen
}

September 30, 2021

\author{
Prepared by \\ OAK RIDGE NATIONAL LABORATORY \\ Oak Ridge, TN 37831-6283 \\ managed by \\ UT-BATTELLE, LLC \\ for the \\ US DEPARTMENT OF ENERGY \\ under contract DE-AC05-00OR22725
}




\section{CONTENTS}

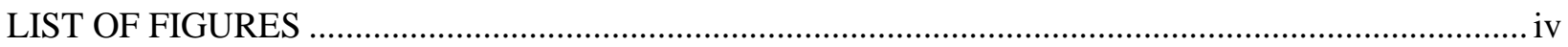

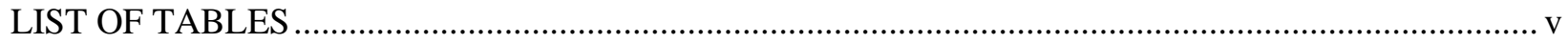

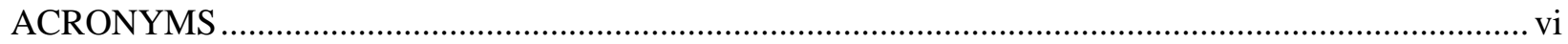

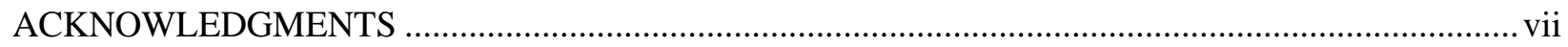

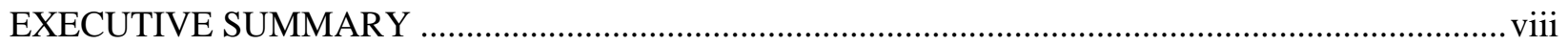

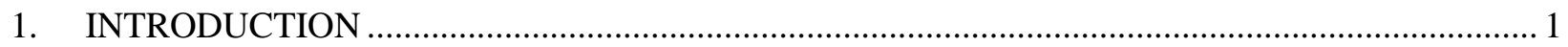

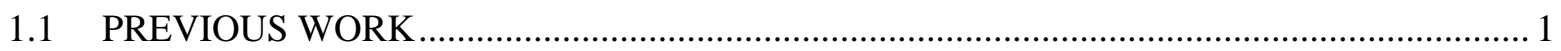

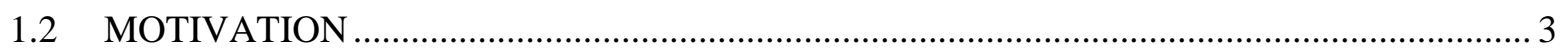

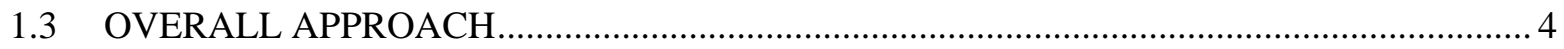

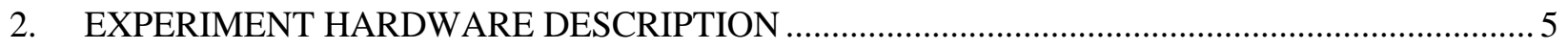

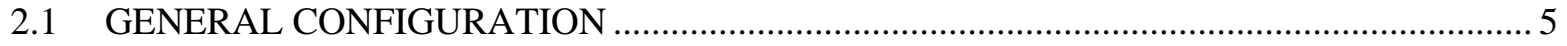

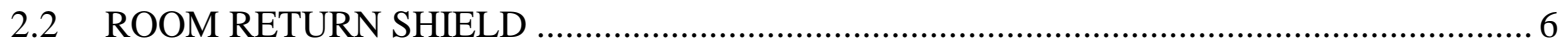

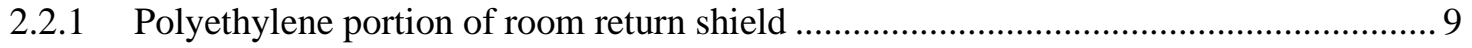

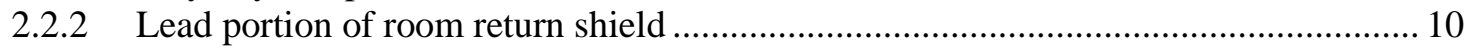

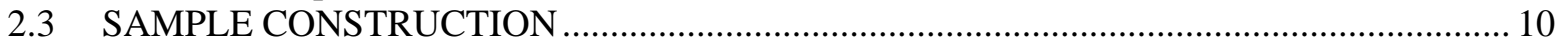

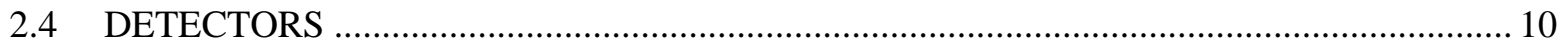

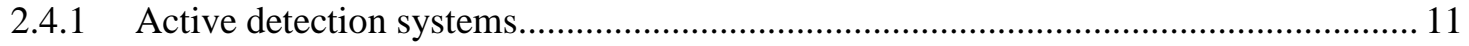

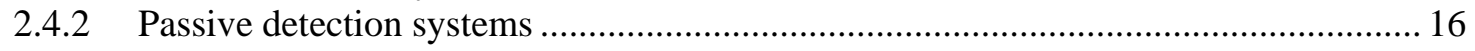

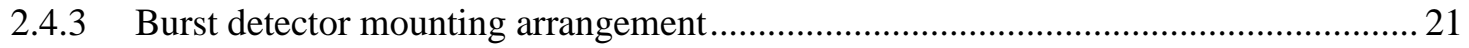

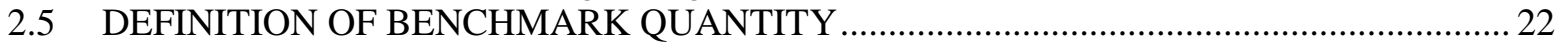

3. FORECASTED MEASUREMENTS AND BENCHMARK QUANTITIES ................................23

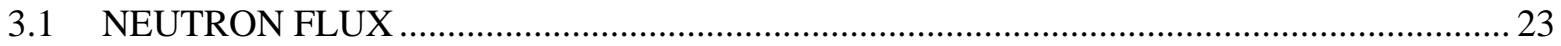

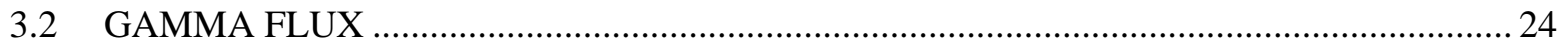

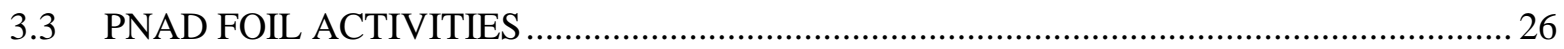

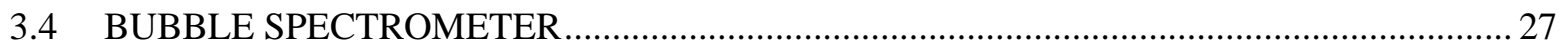

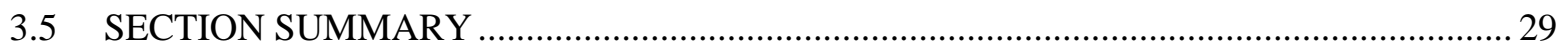

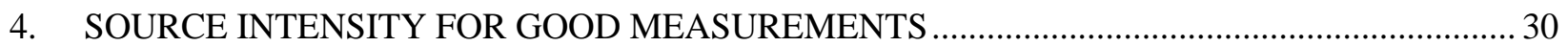

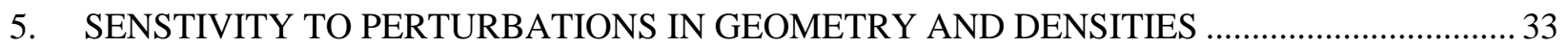

5.1 PERTURBATION SET I: UNCERTAINTIES FAR FROM THE SOURCE ......................... 33

5.1.1 Introduction to Evaluation of Uncertainties Far from the Source .................................. 33

5.1.2 Neutron Results to perturbations far from the source .............................................. 35

5.1.3 Gamma Results to perturbations far from the source............................................. 36

5.2 PERTURBATION SET II: UNCERTAINTIES NEAR THE SOURCE.............................. 38

5.3 UNCERTAINTY OF SOURCE INTENSITY ......................................................... 41

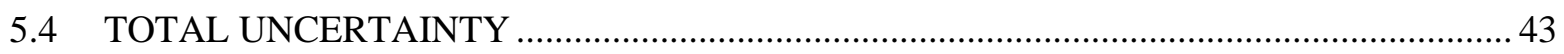

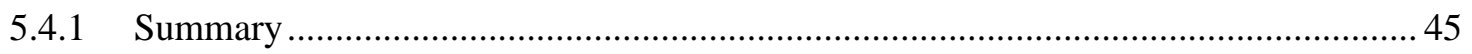

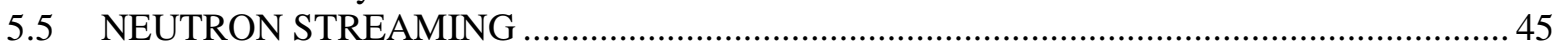

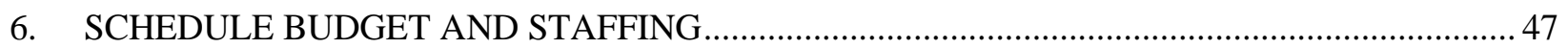

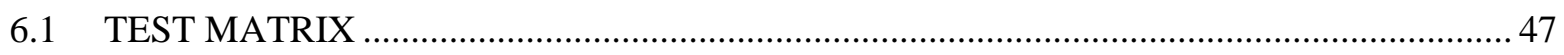

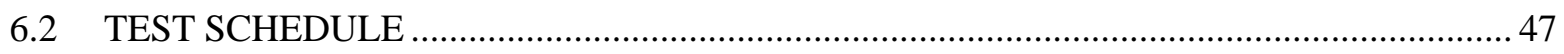

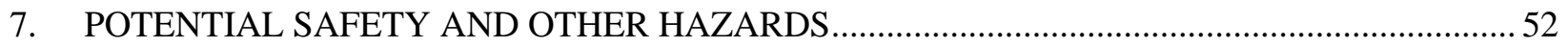

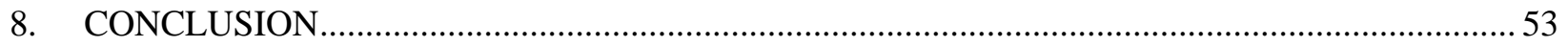

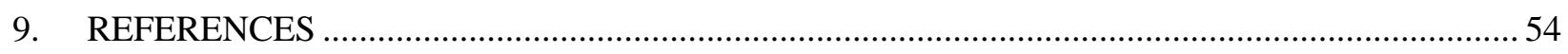

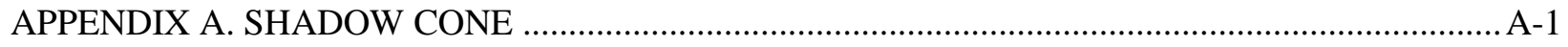

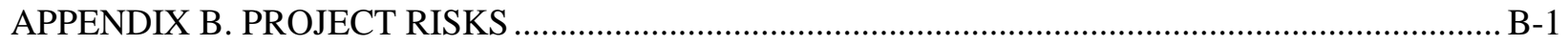

APPENDIX C. EXPERIMENT REQUIREMENTS ….................................................................... 


\section{LIST OF FIGURES}

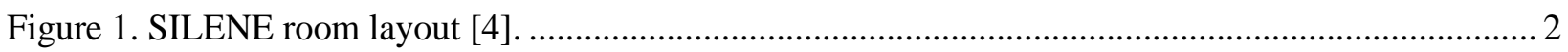

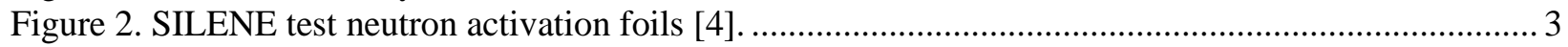

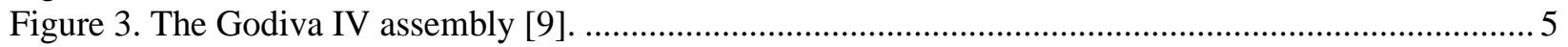

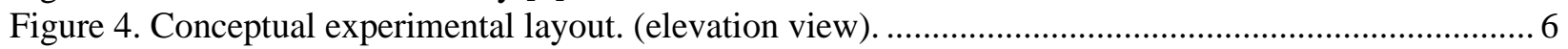

Figure 5. Conceptual rendering of room return shield............................................................. 7

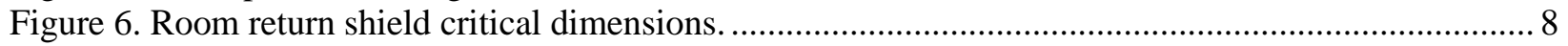

Figure 7. Room return shield cross sections through detectors. …........................................................ 8

Figure 8. Sliding closure concept (sliding rail and rollers not shown). .................................................. 9

Figure 9. Concept of CNC cutouts of overlapping shield pieces to assemble into room return

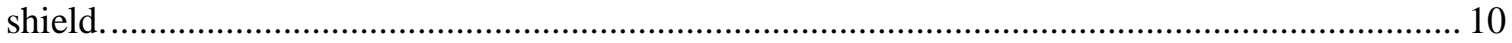

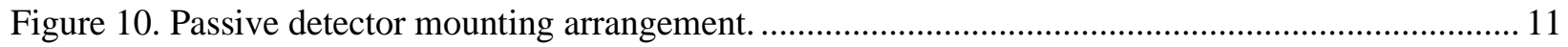

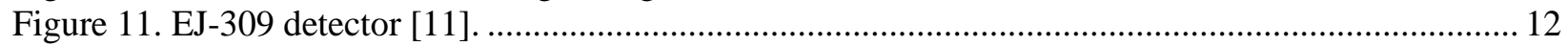

Figure 12. Plot of neutron recoils vs. gamma pulses in the EJ-309 detector

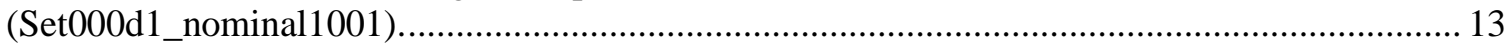

Figure 13. Plot of neutron reactions vs gamma pulses in a 0.2 atm. SP9 detector. ................................. 14

Figure 14. Sample plot of BGO detector response (Courtesy, IRSN). ................................................. 15

Figure 15. Plot of gammas vs. neutron recoils in BGO crystal. Set000d1_nominal1001......................... 16

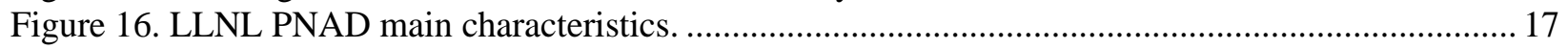

Figure 17. LLNL PNAD foil and other materials characteristics requirements. .................................... 18

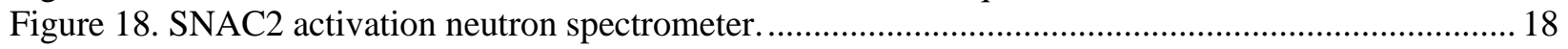

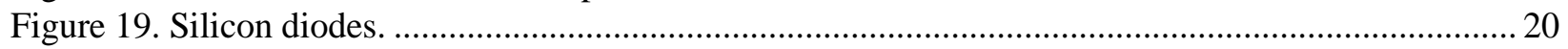

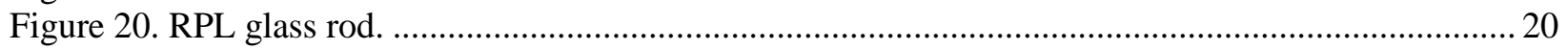

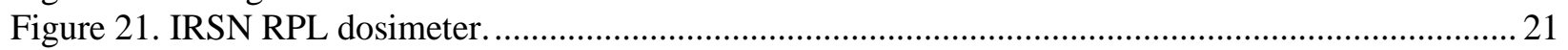

Figure 22. RPL Dose Ace reader for GD 351 glass rod.................................................................... 21

Figure 23. Plot of flux as a fraction of baseline for various shielding materials. .................................... 23

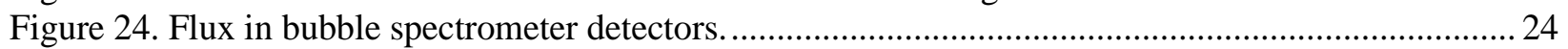

Figure 25. Simulated gamma spectra for various samples............................................................... 25

Figure 26. Gamma fluxes as a fraction of gamma fluxes with no shielding samples............................. 26

Figure 27. MCNP Godiva model (left), MAVRIC model (right)........................................................ 39

Figure 28. Correlation of temperature and reactor period...................................................................... 42

Figure 29. Detector fluxes of simulated opaque shielding samples with no flux trap (orange and yellow at bottom of graph) vs. fluxes for ordinary shielding samples also with no flux

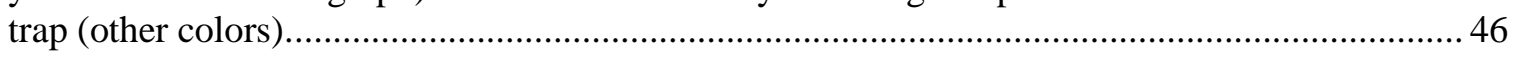

Figure 30. Detector fluxes of simulated opaque shielding sample with no flux trap (orange at bottom of graph) vs fluxes for polyethylene shielding sample (blue). ...................................... 46

Figure 31. Raw data from Table 4 of IER 147 and $1 / \mathrm{r}^{2}$ transmitted doses for a constant room

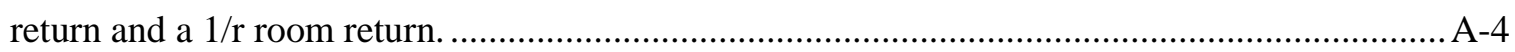

Figure 32. Lightweight gamma-only version of room return shield with CdS liner............................. A-4

Figure 33. Configuration with shadow cone in which only room return is detected.............................. A-5

Figure 34. Configuration without shadow cone in which room return and direct transmission of

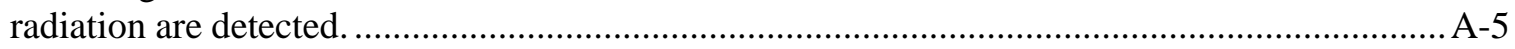

Figure 35. Configuration with room return shield to remove room return while maintaining

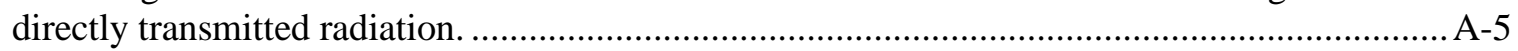

Figure 36. Comparison of room : no-room flux ratio for shadow cone (x-axis) to ratio for room

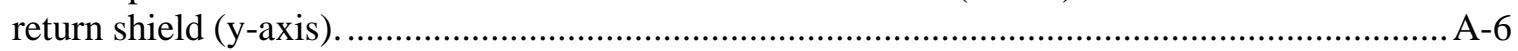

Figure 37. Comparison of room: no-room flux ratio for shadow cone (x-axis) to ratio for room return shield (y-axis) 
Figure 38. Neutron tallies to gauge performance of various room return management strategies for a $\mathrm{SiO}_{2}$ shield sample of $6.2 \mathrm{~cm}$ with Monte Carlo error bars.

Figure 39. Neutron tallies to gauge performance of various room return management strategies for a $\mathrm{SiO}_{2}$ shield sample of $6.2 \mathrm{~cm}$ and measurement errors of $20 \%$.

Figure 40. Gamma tallies to gauge performance of various room return management strategies for a $\mathrm{SiO}_{2}$ shield sample of $6.2 \mathrm{~cm}$ and Monte Carlo error bars.....

Figure 41. Gamma tallies to gauge performance of various room return management strategies for a $\mathrm{SiO}_{2}$ shield sample of $6.2 \mathrm{~cm}$ and measurement errors of $20 \%$.

\section{LIST OF TABLES}

Table 1. Foil activities $(\mathrm{Bq})$ for $10 \mathrm{~W}$ source power for 4,000 seconds with three hours of decay............27

Table 2. Foil activities $(\mathrm{Bq})$ for $70^{\circ} \mathrm{C}$ burst after 3 hours of decay .................................................... 27

Table 3. Neutron dose for various energy thresholds, bubble production rates, and number of

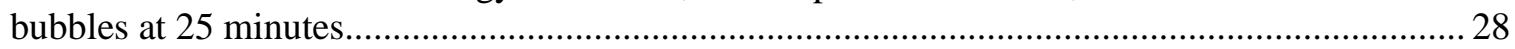

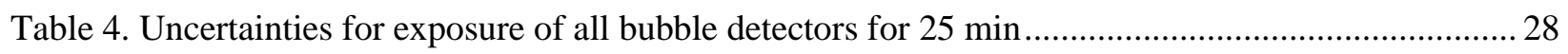

Table 5. Detector performance range and corresponding source intensity ........................................... 30

Table 6. Detector performance ranges and corresponding source intensity ............................................. 31

Table 7. Times to obtain a workable reading at various power levels (or number of bursts

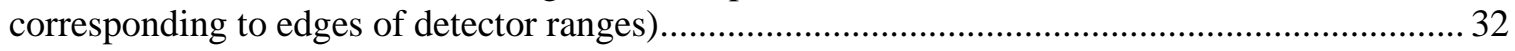

Table 8. Overview of geometry and density perturbations performed with scaling multipliers................. 34

Table 9. Fast neutron detector tally sensitivities to far-from-source perturbations .................................... 36

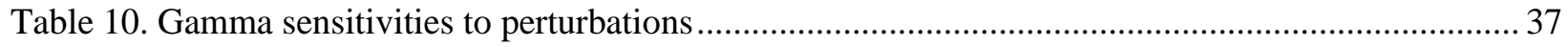

Table 11. Perturbations of geometry and density near the source …....................................................... 39

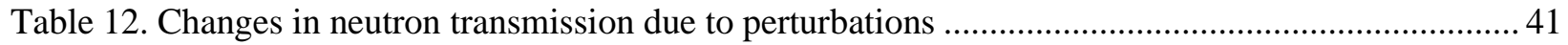

Table 13. Changes in gamma transmission due to perturbations ............................................................... 41

Table 14. Hypothetical uncertainty for two measurement neutron benchmark quantity.......................... 44

Table 15. Hypothetical uncertainty for single measurement neutron benchmark quantity........................ 44

Table 16. Potential uncertainty for two measurement gamma benchmark quantity .................................. 45

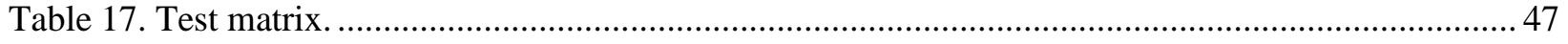

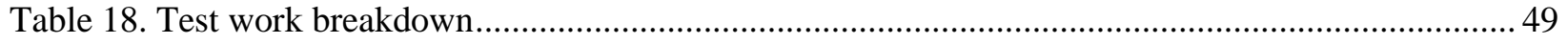

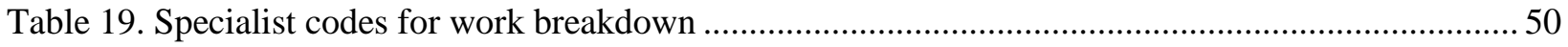

Table 20. Detailed schedule (orange rows indicate trip to count lab) ..................................................... 50

Table 21. Rough, order of magnitude cost and acquisition time estimates............................................ 51 


\section{ACRONYMS}

\begin{tabular}{|c|c|}
\hline ALARA & as low as reasonably achievable \\
\hline BEIR & Biologic Effects of Ionizing Radiation \\
\hline BGO & bismuth germanate \\
\hline CAAS & criticality accident alarm system \\
\hline $\mathrm{C} / \mathrm{E}$ & calculated-to-experimental \\
\hline CED-2 & Critical/Subcritical Experiment Decision 2 \\
\hline $\mathrm{CNC}$ & computer numerical control \\
\hline HEU & highly enriched uranium \\
\hline ICPMS & inductively coupled plasma mass spectrometry \\
\hline ICSBEP & International Criticality Safety Benchmark Evaluation Project \\
\hline IEEE & Institute of Electrical and Electronics Engineers \\
\hline IER & Integral Experiment Request \\
\hline IRSN & French Institut de Radioprotection et de Sûreté Nucléaire \\
\hline LANL & Los Alamos National Laboratory \\
\hline LLNL & Lawrence Livermore National Laboratory \\
\hline NCERC & National Critical Experiments Research Center \\
\hline NCSP & Nuclear Criticality Safety Program \\
\hline NNSS & Nevada National Security Site \\
\hline ORNL & Oak Ridge National Laboratory \\
\hline PNAD & personal nuclear accident dosimeter \\
\hline PSD & pulse shape discrimination \\
\hline RPL & radiophotoluminescence \\
\hline SPR-III & Sandia Pulsed Reactor III \\
\hline $\mathrm{S} / \mathrm{U}$ & sensitivity and uncertainty \\
\hline TLD & thermoluminescent dosimeter \\
\hline
\end{tabular}




\section{ACKNOWLEDGMENTS}

This work was supported by the Nuclear Criticality Safety Program, funded and managed by the National Nuclear Security Administration for the US Department of Energy.

The Critical \& Subcritical Experiment Design Team $\left(\mathrm{C}_{\mathrm{E}} \mathrm{DT}\right)$ responsible for Integral Experiment Request 498 was instrumental in completing this report. Members include:

$\begin{array}{lll}\text { Joetta Goda } & \text { Experiment Member } & \text { Los Alamos National Laboratory } \\ \text { Catherine Percher } & \text { Member } & \text { Lawrence Livermore National Laboratory } \\ \text { Bill Myers } & \text { Member } & \text { Los Alamos National Laboratory } \\ \text { Michael Zerkle } & \text { NDAG Member } & \text { Naval Nuclear Laboratory } \\ \text { Cihangir Celik } & \text { Methods Member } & \text { Oak Ridge National Laboratory } \\ \text { Riley Cumberland } & \text { C }_{\mathrm{E}} \text { DT Lead } & \text { Oak Ridge National Laboratory }\end{array}$

contributors who provided insights and advice include:

\begin{tabular}{|c|c|}
\hline Mathieu Dupont & Collaborator \\
\hline Tom McLean & Collaborator \\
\hline Francois Trompier & Collaborator \\
\hline Robert Weldon & Collaborator \\
\hline Chris Romero & Collaborator \\
\hline Travis Grove & Collaborator \\
\hline Daniel Stone & Collaborator \\
\hline Matthieu Duluc & Collaborator \\
\hline David Heinrichs & Collaborator \\
\hline Theresa Cutler & Collaborator \\
\hline Arzu Alpan & Reviewer \\
\hline Charles Daily & Reviewer \\
\hline Rose Raney & Editor \\
\hline Robert Kimpland & Editor \\
\hline
\end{tabular}

Oak Ridge National Laboratory

Los Alamos National Laboratory

Institut de Radioprotection et de Sûreté Nucléaire

Los Alamos National Laboratory

Los Alamos National Laboratory

Los Alamos National Laboratory

Lawrence Livermore National Laboratory

Institut de Radioprotection et de Sûreté Nucléaire

Lawrence Livermore National Laboratory

Los Alamos National Laboratory

Oak Ridge National Laboratory

Oak Ridge National Laboratory

Oak Ridge National Laboratory

Los Alamos National Laboratory 


\section{EXECUTIVE SUMMARY}

This report supports Critical/Subcritical Experiment Decision 2 (CED-2) for Integral Experiment Request (IER) 498, a shielding benchmark produced using the Godiva IV critical assembly at the National Critical Experiments Research Center (NCERC). Shielding benchmarks are datasets collected to provide a comparison for computer models that simulate the effect of shielding on radiation transmission from a source to a detector. The purpose of a CED-2 report is to (1) show uncertainty is sufficiently low for the benchmark test to proceed, and (2) produce a design of sufficient detail to support procurement and final design for an integral experiment and to identify the long lead-time items [1].

Benchmarks for criticality accident alarm system (CAAS) shielding help ensure confidence in models of criticality accident consequences. These improved models can support effective CAAS detector placement when criticality accidents may result in dose to personnel, and they enable more accurate analysis of possible criticality accident impacts. The second programmatic need supported by the shielding benchmarks is the ongoing development of sensitivity and uncertainty ( $\mathrm{S} / \mathrm{U})$ methods and data integration into nuclear simulation codes such as SCALE. The benchmarks may be useful to S/U method developers in efforts funded by the Nuclear Criticality Safety Program (NCSP).

The range of uncertainties associated with the modeling of a shielding system includes physical dimensions, nuclear data, uncertainty in composition, uncharacterized heterogeneity, and others. While many of these uncertainties can be quantified in simulation space, it is beneficial to validate them using results from experimental work to ensure that they are being accurately represented. Because shielding problems are highly dimensional, multiple benchmarks are necessary and should include a diverse set of material combinations and geometric configurations. The Godiva IV assembly's fast spectrum and variable intensity make it suitable to support this range of needs.

A shielding benchmark fundamentally consists of a source, some transmission path for the radiation, and a detector. The source discussed herein will be the Godiva IV assembly. The transmission pathway will be through the air between the source and the detectors as well as through a shielding sample. The assembly is in a relatively small room that contains other equipment. Therefore, this work will require effective isolation of the measurements from the scattering that occurs in the room itself, as well as scattering from the other components in the room. The detector and shielding sample will be in a 2-ton cylinder to limit the field of view of the shielding sample and detectors to the source. This ensures that radiation scattering off the room walls or other hardware in the room is not detected. In this report, the cylinder is referred to as a room return shield.

This document details an experimental configuration designed to obtain shielding benchmark data from the Godiva IV critical assembly. The document builds upon the IER 498 CED-1 report to provide a design and measurement plan by which data can be obtained to support a benchmark. Various detectors are employed to enable cross comparisons between similar measurements. Uncertainties from geometry and material densities are considered to ensure that uncertainty is controlled. The requirements are reviewed below, followed by descriptions of workable experimental configurations. Finally, a preliminary schedule for development and installation of the experiment is outlined.

The level of detail for the room return concept in this report will ensure that a designer and a mechanical engineer can work with vendors to produce a working product. The nuclear design is complete, but some mechanical and structural items remain unspecified. The room return shield geometry and compositions are specified, and some possible means of production are discussed; however, the vendors and those working with the vendors have substantial flexibility in how to produce the product. 
Fast neutron detectors and gamma detectors were selected. These are sourced from Los Alamos National Laboratory (LANL) and French Institut de Radioprotection et de Sûreté Nucléaire (IRSN). Foils to capture burst information included in the Lawrence Livermore National Laboratory (LLNL)-supplied personal nuclear accident dosimeter (PNAD) are modeled. CaF2 thermoluminescent dosimeter (TLD), and IRSN-supplied radiophotoluminescence (RPL) glass can provide integrated gamma measurements. The apparatus was modeled to produce order of magnitude estimates of source levels at which each detector is sensitive to produce an experiment plan. Also, a sensitivity study with 21 different perturbations was performed to ensure that the test will be stable to geometry and density changes. These provide a starting point to the CED-4 evaluation to produce an International Criticality Safety Benchmark Evaluation Project (ICSBEP) evaluation.

An experiment scheduled over the course of three weeks will be developed to support detailed planning in CED-3A. After receiving the equipment, the first week of the effort will be devoted to setting up detectors, aligning the room return shield, and performing first measurements with every measurement system. The second week of tests will consist of measurements with passive detectors at steady state, followed by electronic detectors at steady state. The second week may not immediately follow the first week. The final week will consist of burst testing the shielding samples.

This report describes the design for a series of CAAS benchmark measurements in the fast spectrum using the Godiva IV as a source. It outlines the studies to be performed to ensure that the design functions as intended. The CAAS benchmark should produce results of sufficient quality to support shielding model validation. Furthermore, it should produce a piece of equipment that can be used for multiple follow-on experiments layering different materials, using different detectors, and using different sources.

Before devoting substantial investment in experiment execution, further work is needed for the team to ensure that the goals of this IER can be sufficiently satisfied. Specifically, the Godiva IV source intensity should be verified to be either reproducible or recordable with uncertainty sufficiently small to support the benchmark. Nominally a 5\% one sigma uncertainty in source intensity should be sufficient, but this value could change. Sufficient study likely requires some type of experimental work in a separate IER. 


\section{INTRODUCTION}

This report supports Critical/Subcritical Experiment Decision 2 (CED-2) for Integral Experiment Request (IER)-498, a shielding benchmark dataset produced using the Godiva IV critical assembly at the National Critical Experiments Research Center (NCERC). Shielding benchmarks are datasets documenting a radiation source, transmission media such as air or lead, the geometry of the transmission media, and a radiation measurement. Once established, a benchmark can be used to ensure the quality of shielding simulations through validation using a process such as that outlined in the Institute of Electrical and Electronics Engineers (IEEE) standard IEEE P1012 [2].

Development of criticality accident alarm system (CAAS) shielding benchmarks supports two main programmatic needs. The first need is to improve confidence in results from models of facilities where nuclear materials are handled. The improved confidence can support effective CAAS detector placement when criticality accidents may result in dose to personnel, and they can also enable more accurate analysis of possible criticality accident impacts at various locations in the facility. This information is then used to plan responses to the accident. The second programmatic need supported by shielding benchmarks is the ongoing development of sensitivity and uncertainty $(\mathrm{S} / \mathrm{U})$ methods and data being integrated into nuclear simulation codes such as SCALE and MCNP. The benchmarks may be useful to S/U method developers in the efforts funded by the Nuclear Criticality Safety Program (NCSP). Models of secondary gamma production (resulting from neutron absorption and inelastic scattering) may benefit from validation against a benchmark.

This document details an experimental configuration designed to obtain shielding benchmark data from the Godiva IV critical assembly. The document builds upon the IER 498 CED-1 report to produce a design and measurement plan to obtain data to support a benchmark. A variety of detectors will be employed to enable cross comparisons between similar measurements. Uncertainties from geometry and material densities are considered to ensure that overall uncertainty is controlled. The requirements are reviewed herein, followed by a description of a workable experimental configurations. Finally, a preliminary schedule for development and installation of the experiment is outlined.

\subsection{PREVIOUS WORK}

Numerous shielding benchmark datasets have been developed in the past. The Organisation for Economic Co-operation and Development Nuclear Energy Agency (OECD-NEA) has collected many of these into the Shielding Integral Benchmark Archive Database (SINBAD) [3]. Furthermore, the International Criticality Safety Benchmark Evaluation Project (ICSBEP) has a more select set of benchmarks. This includes a set of tests performed using the SILENE assembly at the Valduc facility in France to produce a shielding benchmark for a pulse of a solution system. The SINBAD benchmarks tend to lack the documentation desired for this effort, and they are limited to approximately a dozen fast shielding benchmarks. Software developers need shielding benchmark datasets to confirm the ability to properly model physical systems. The range of uncertainties associated with the modeling of a shielding system includes physical dimensions, nuclear data, uncertainty in composition, uncharacterized heterogeneity, and others. While many of these uncertainties can be quantified in simulation space, it is beneficial to validate these uncertainties using results from experimental work to ensure that they are being accurately represented. Because shielding problems are highly dimensional, multiple benchmarks are necessary and should include a diverse set of material combinations and geometric configurations. The Godiva IV assembly's fast spectrum and variable intensity make it well suited to support this range of needs.

Perhaps the work that best informs the current efforts is a set of shielding benchmark tests performed using the SILENE critical assembly at the Valduc facility in France $[4,5,6]$. It constitutes three of the four shielding benchmarks in the ICSBEP handbook. The critical assembly consisted of a uranyl nitrate 
solution and a pulsing rod to enable prompt supercritical pulses. The shielding experiments at Valduc evaluated three assembly pulse configurations: bare, reflected/shielded with polyethylene, and reflected/shielded with lead. The overall approach was to install shielding material close to the assembly and to take measurements far away in collimator boxes to reduce room return. The facility had an open floorplan.

Several principal uncertainties dominated the experiment source strength uncertainty which was fixed at $6 \%$, and measurement uncertainty ( $2 \%$ or more). Uncertainty in concrete composition was a perennial problem for the investigators. In two of the three experiments, concrete was directly in the path of the radiation for two of the three measurement locations. Furthermore, the room was constructed of concrete. Concrete absorbs and desorbs moisture, and it has a variable isotopic composition, depending upon the aggregate used. These factors result in a substantial source of uncertainty.

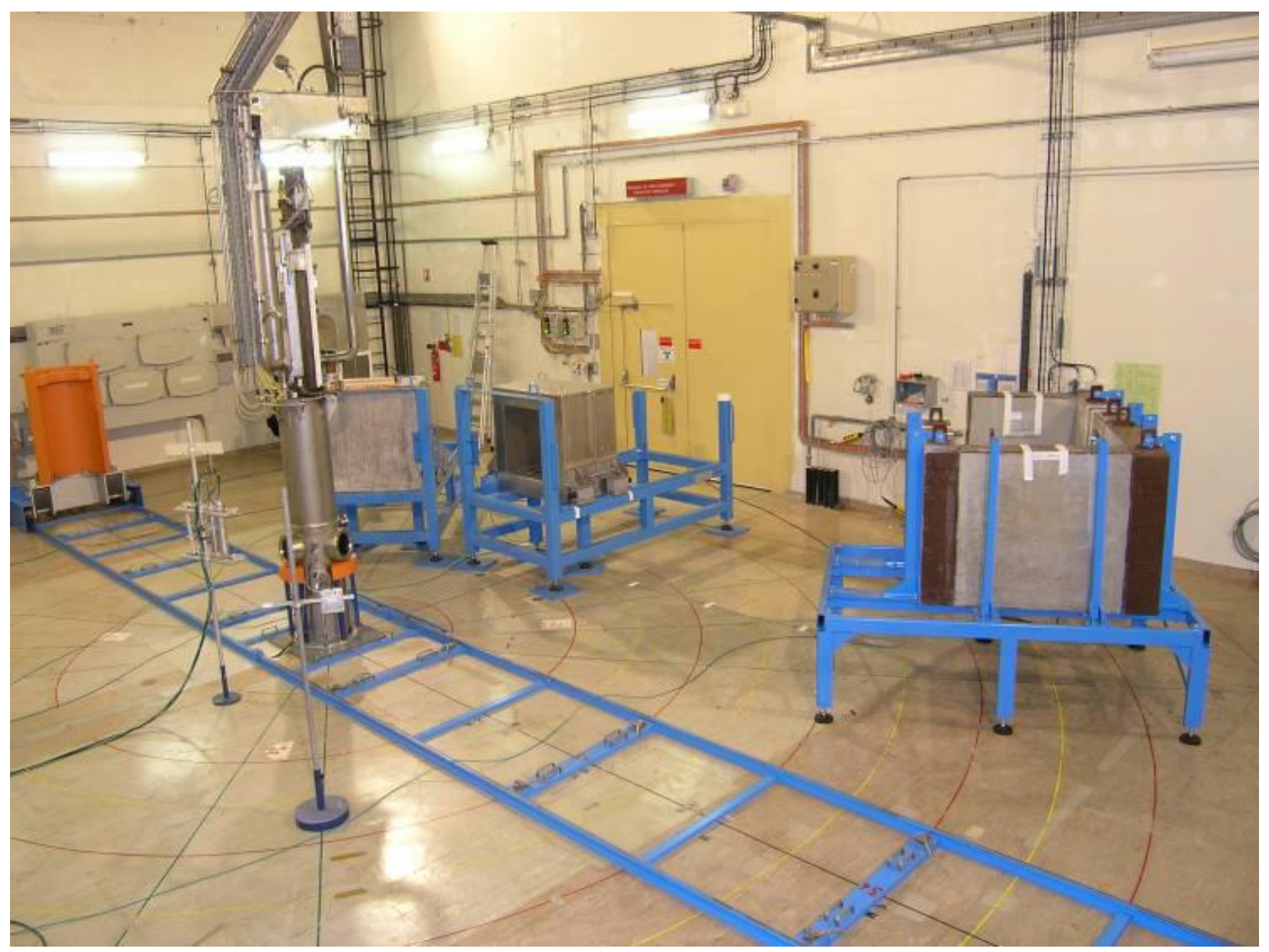

Figure 1. SILENE room layout [4].

The uncertainty in the number of fissions produced during the pulse was estimated to be about $5 \%$, partly because the detection equipment's uncertainty had not been measured [6, p. 83]. Moreover, the assembly's variable fluid level [7, pp. 38-39] introduced additional uncertainty in the proportion of radiation transmitted from the assembly to the instrumentation. 


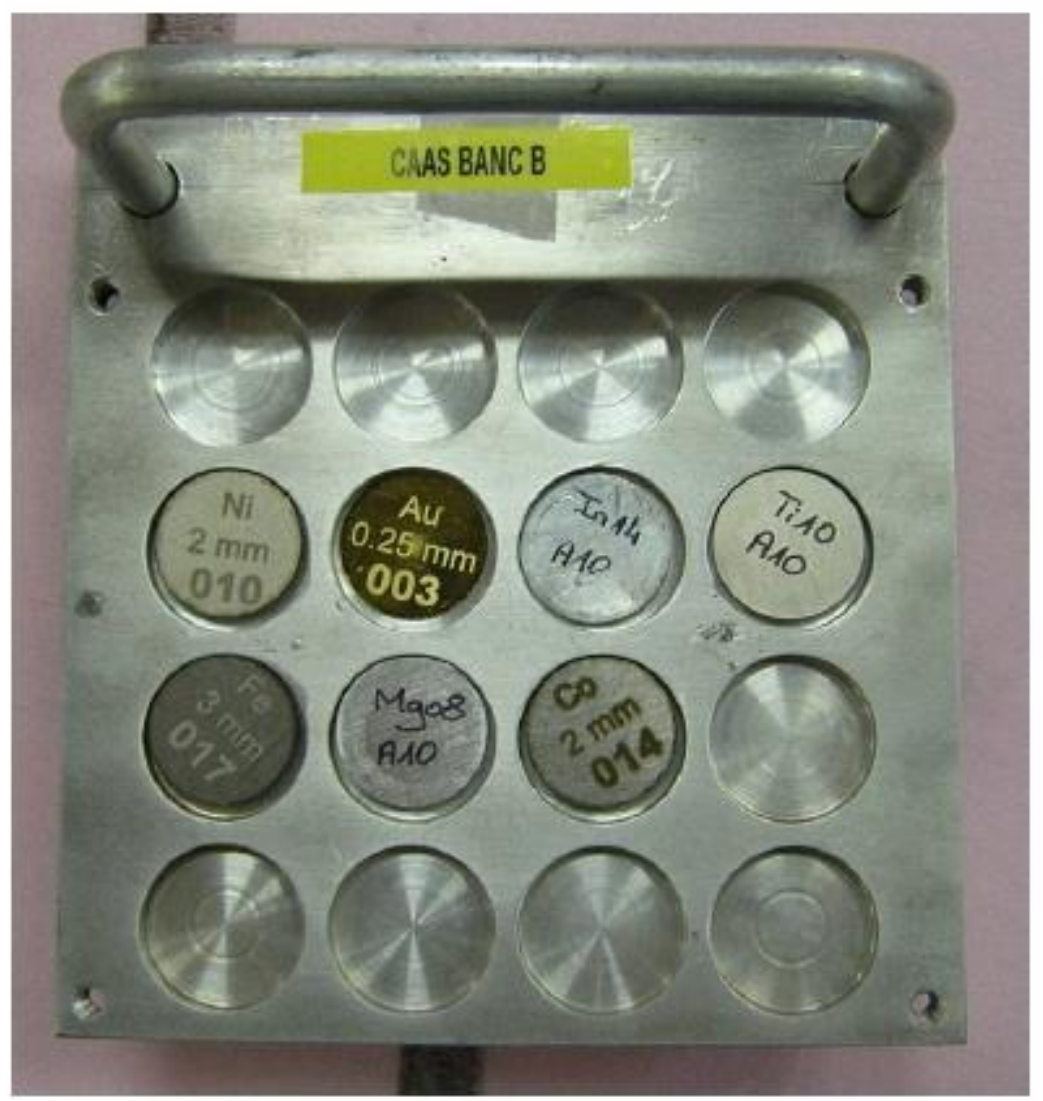

Figure 2. SILENE test neutron activation foils [4].

Finally, because of the speed of the pulse, detectors were restricted to foil activations and thermoluminescent dosimeter (TLD) measurements. Two-sigma confidence intervals for activation foils hovered at around 4\%, and two-sigma confidence intervals for TLDs hovered at around 6\% [5, p. 71] [4, p. 80]. Because secondary photon production is a key piece of data for evaluating doses from criticality accidents, the accurate recording of gamma-related spectral information is a high-priority goal that was not achieved during the Silene experiments.

\subsection{MOTIVATION}

The objective for establishing the proposed benchmark is to support validation of shielding models of fast systems. For an integrated experiment, the pure, interference-free simplicity of a calibration facility is only ideal in select circumstances. Some integrated experiments should have some level of complexity to begin to approximate a real-world scenario for which a code may be used. At the same time, the experiment should remain simple and well-instrumented enough to be able to facilitate tracing of differences between a code result and the experimental measurement.

The work for the proposed benchmark would be performed at NCERC at the Nevada National Security Site (NNSS) using the Godiva IV assembly based on (1) its ability to replicate a criticality accident of a fast metal system and (2) its source intensity, both of which enable timely collection of data. The assembly can be operated as a source of neutrons in various regimes of criticality (i.e., super-prompt supercritical, at delayed critical at various power levels spanning more than 6 orders of magnitude, or it can be used to provide a subcritical configuration at various neutron multiplications. 
The technical goal of this work is to produce robust shielding benchmark data of sufficient precision to support benchmarking to validate physical models that predict dose received from a criticality accident. For shielding purposes, dose rate uncertainty on the order of $20 \%$ is understood to be acceptable given the many unknowns of radiation transmission and the exponential nature of radiation attenuation. Previous work has been able to provide uncertainties at approximately $6 \%$ to $11 \%$ depending on the measurement $[4,5,6]$. Because the underlying $95 \%$ confidence interval in the biological effects of radiation is approximately 20-50\% (as presented in the risk models in Tables 12-1 and 12-2 of the Biologic Effects of Ionizing Radiation [BEIR] VII report [8]), a 95\% confidence interval on the order of $20 \%$ in measured dose for a given source intensity is likely sufficient.

\subsection{OVERALL APPROACH}

A shielding benchmark fundamentally consists of a source, some transmission path for the radiation, and a detector. As discussed here, the source will be the Godiva IV assembly. The transmission pathway will be through the air between the source and the detectors, as well as through a shielding sample. The detector and shielding sample will be in a cylinder to limit the field of view of the shielding sample and detectors to the source. This will prevent detection of radiation scattering off the room walls or other hardware in the room. The internals of the room return shield do interact with the radiation transmission, but this is an integrated benchmark, so it is acceptable given that the materials are known. It is a fast benchmark, so lack of resolution caused by this in the intermediate energy ranges is acceptable.

Furthermore, it is difficult if not impossible to obtain spectroscopic information on the intermediate region. The more common method of using a shadow cone to determine room return was explored, as described in an appendix. 


\section{EXPERIMENT HARDWARE DESCRIPTION}

\subsection{GENERAL CONFIGURATION}

The overall configuration of the shielding benchmark experiment consists of the Godiva IV assembly (Figure 3 and left of center in Figure 4) and a room return shield (purple object on right of center in Figure 4). The room return shield is open on one side to allow radiation from the Godiva IV assembly to enter and to exclude any scatter from the concrete room.

The Godiva IV assembly can produce a wide range of neutron intensities on the order of a strong californium source up to burst intensities of $10^{17}$ neutrons. The assembly is described in detail in the HEU-MET-FAST-086 [9] benchmark specification.

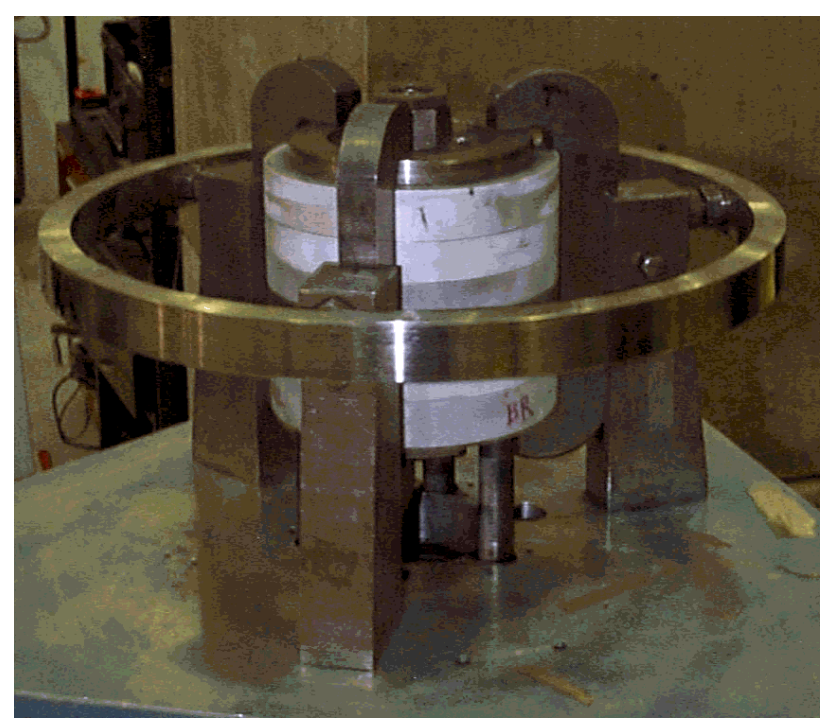

Figure 3. The Godiva IV assembly [9].

A variety of shielding samples can be placed inside the room return shield to create a variety of benchmark configurations. Additionally, lead can be placed between the sample and the source to block primary gamma rays. Alternatively, polyethylene can be placed between the source and the shielding sample to produce a more thermalized spectrum. These options are not explored in this IER, but they may appear in follow-up proposals.

Detectors are located in the room return shield. These can include electronic detectors, as well as integral detectors such as bubble dosimeters, activation foils, glass dosimeters, etc. A key objective of this analysis is to identify power levels suitable for measurements of shielding. Detector cabling is routed through a cable channel that nominally follows the contour of the clamshell closure. 


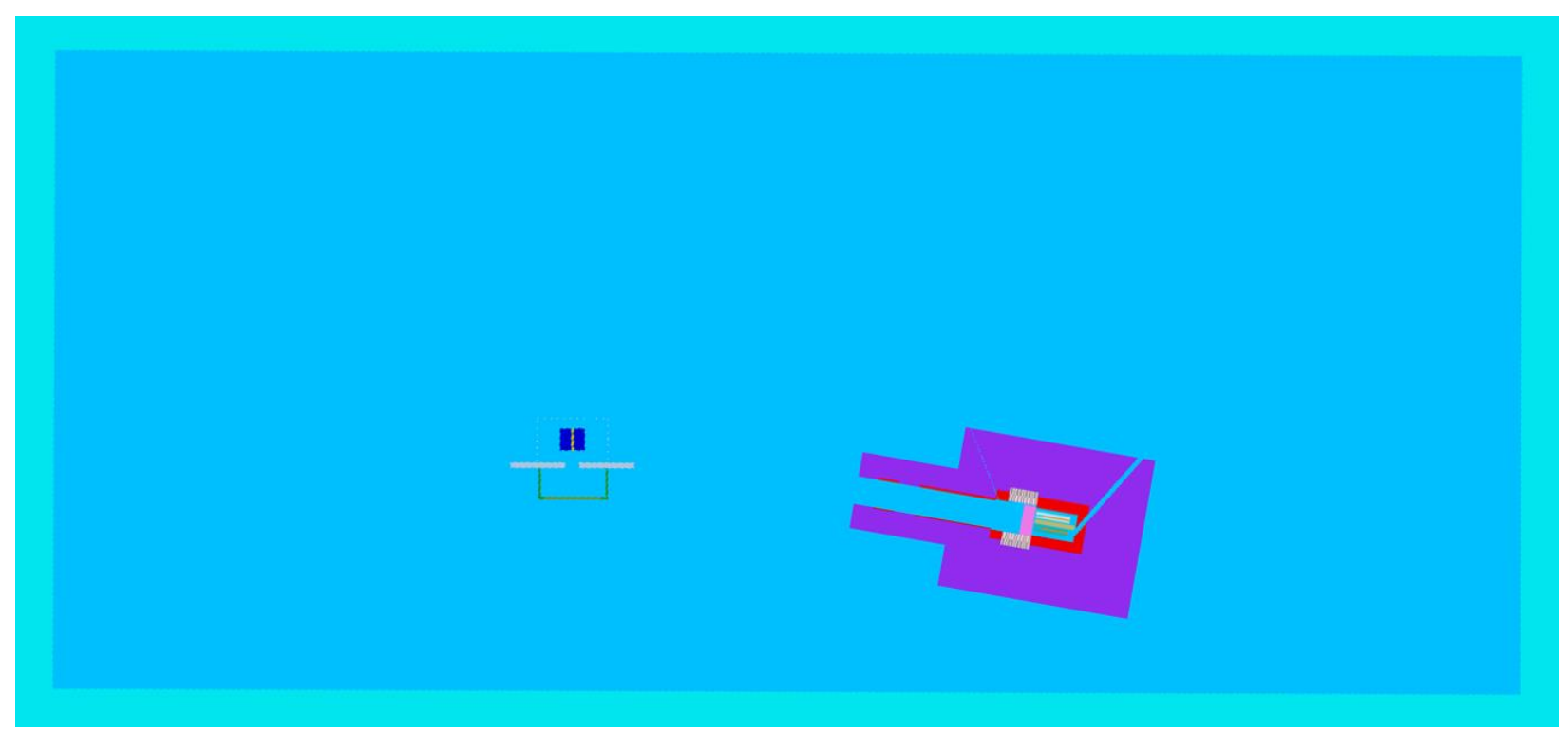

Figure 4. Conceptual experimental layout. (elevation view).

\subsection{ROOM RETURN SHIELD}

A conceptual drawing of the room return shield is shown in Figure 5 below. The steel support structure will be developed further to ensure that the room return shield can pivot and be transported through the facility. The dimensions critical for the experiment are listed in this section, and the dimensions for the support frame are to be developed later, possibly by the fabricator.

For reproducibility, a laser sighting mechanism, locking wheels, and floor markings are recommended. Use of an indoor laser distance measuring tool can provide distance information precise to $2 \mathrm{~cm}$. Using a location in the room that was also used for dosimetry measurements during IER147 may be advantageous. The room return shield should fit through the doorways leading to the room. An envelope no greater than 6 feet in width and 6 feet in height should be a sufficient limit for this. The concept is not expected to approach this envelope. 


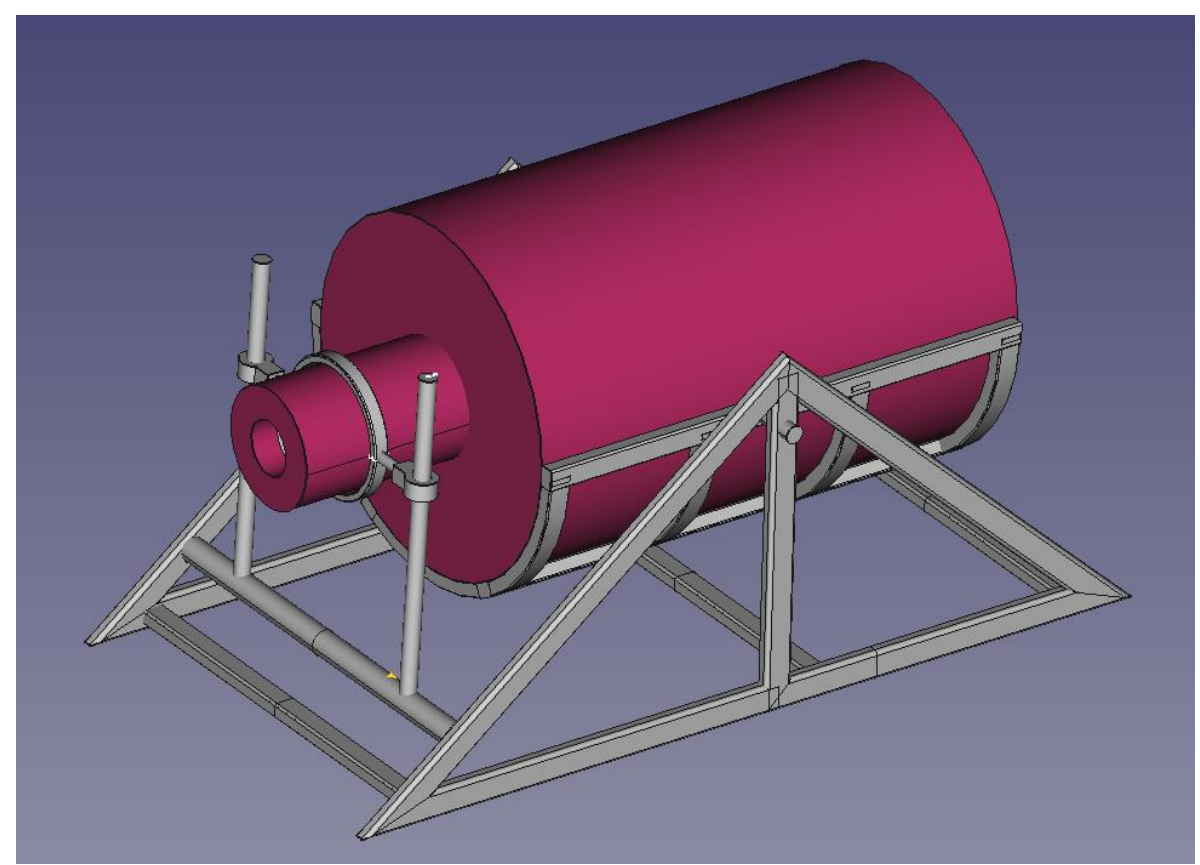

Figure 5. Conceptual rendering of room return shield.

Figure 6 shows key dimensions of the room return shield. The cylindrical room return shield is composed of $5 \%$ borated polyethylene (purple), and powder coated lead (red). The blue portion of the diagram represents air. The shielding sample which can be changed between tests is pink. Approximations of detector electronics are orange, and sleeves for detectors are also shown. Because the slice for the cross section is down the centerline, the detectors themselves are not shown. All parts of the room return shield are cylindrical and are concentric with the same axis. The bore has a region of slightly increased size to house the shielding sample, which could weigh hundreds of pounds if composed of lead or tungsten. The detectors are not entirely in the view of the cut plane for Figure 6, but the cut plane does intersect the orange cylinders which are approximations of photomultiplier tubes. The detectors are housed in shielded shells to reduce measurement of backscattered radiation. The detector and shielding samples are accessed from the top using a sliding closure mechanism. The cables for the detector enter the room return shield via a cable channel. These are shown in the figure as a stylized worst-case in terms of radiation. In the design, they should be kinked to prevent a streaming path for radiation. The room return shield is expected to be mounted on a tiltable steel frame that can be rolled into and out of the room. Tilting will be accomplished with hydraulics or screw jacks. 


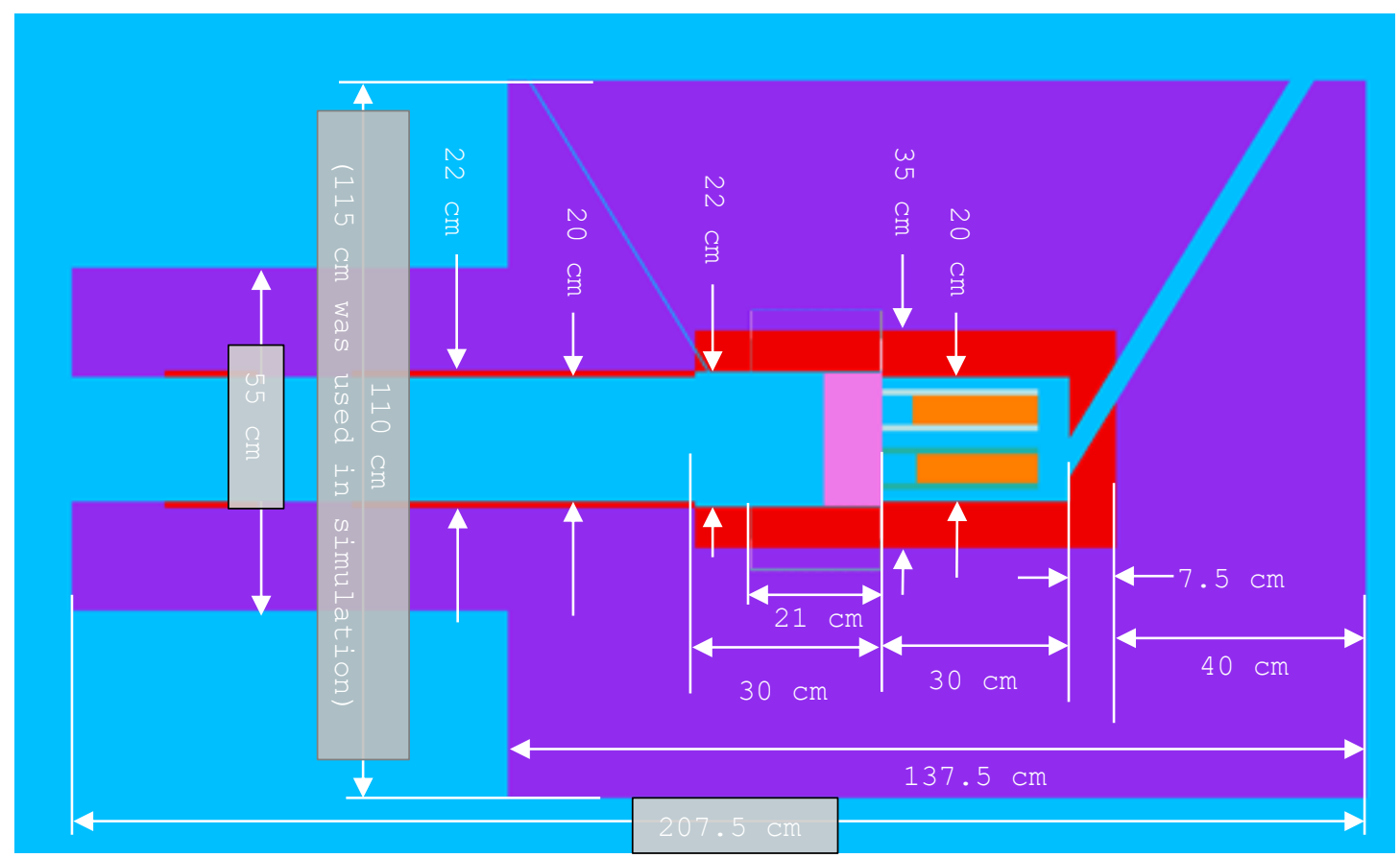

Figure 6. Room return shield critical dimensions.

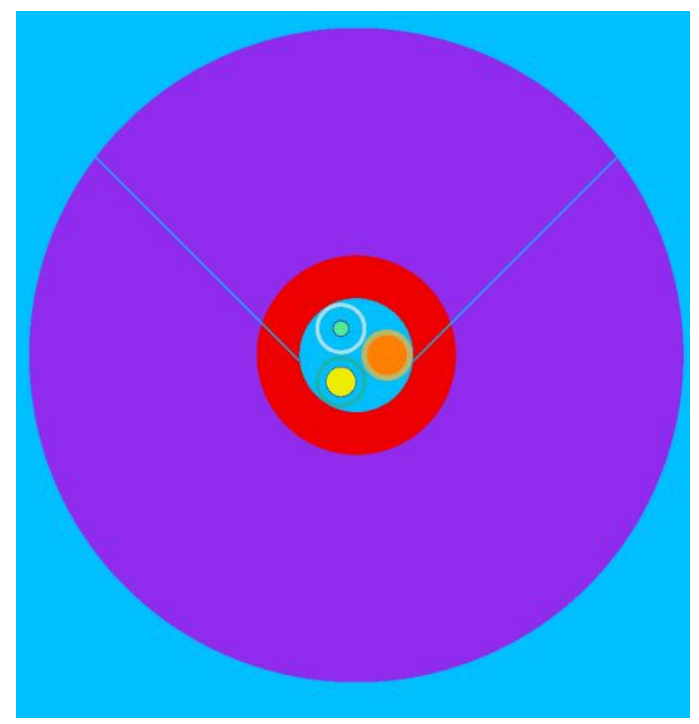

Figure 7. Room return shield cross sections through detectors.

The concentricity of lead parts and the inside of the polyethylene cylinder should be to within $1 \mathrm{~mm}$. The bore of the lead cylinder should be cylindrical to within $1 \mathrm{~mm}$. The composition and mass of the cylinder should be measured prior to assembly and usage to ensure that models are accurate. Composition measurement may be performed using inductively coupled plasma mass spectrometry (ICPMS) or alloy specification. Composition measurements should identify constituents that are at least $0.1 \%$ of the total part mass. The powder coating of the lead shield is expected to be a thin $0.1 \mathrm{~mm}$ layer principally composed of hydrocarbons, so it should not affect the benchmark. ICPMS will be performed on a powder coat sample prior to powder coating to verify that no boron, cadmium, erbium, or gadolinium is present and to obtain a nominal composition. Epoxy adhesives should be avoided in the room return shield because the compositions are not known and often proprietary. 
The room return shield could have a sliding type closure mechanism roughly illustrated in Figure 8 . It is highly preferable that the cut for closure be stair-stepped in the polyethylene and lead areas to prevent radiation streaming. The sliding portion should be long enough to facilitate removal and insertion of detectors and the shield sample (pink in the figure). The shield sample may weigh hundreds of pounds if it is made of lead or tungsten metal. In the figure below, no stairstep was simulated, and the closure lid was conservatively assumed to be a wedge shape without a stairstep to facilitate modeling. The closure should be adjacent to the cable channel for easy placement and removal of cabling to the detectors. In the closure, the air gap should be under $2 \mathrm{~mm}$. In the sliding portion, polyethylene can be slid onto the lead with notches and grooves to keep it together (not shown). Also, the rail and framing to facilitate sliding is not shown. A pair of screw jacks could accomplish the task of opening. Figure 8 also shows a flux trap in the design to prevent streaming around some samples. Ability to easily modify the room return shield to add a flux trap should be considered when producing fabrication drawings.

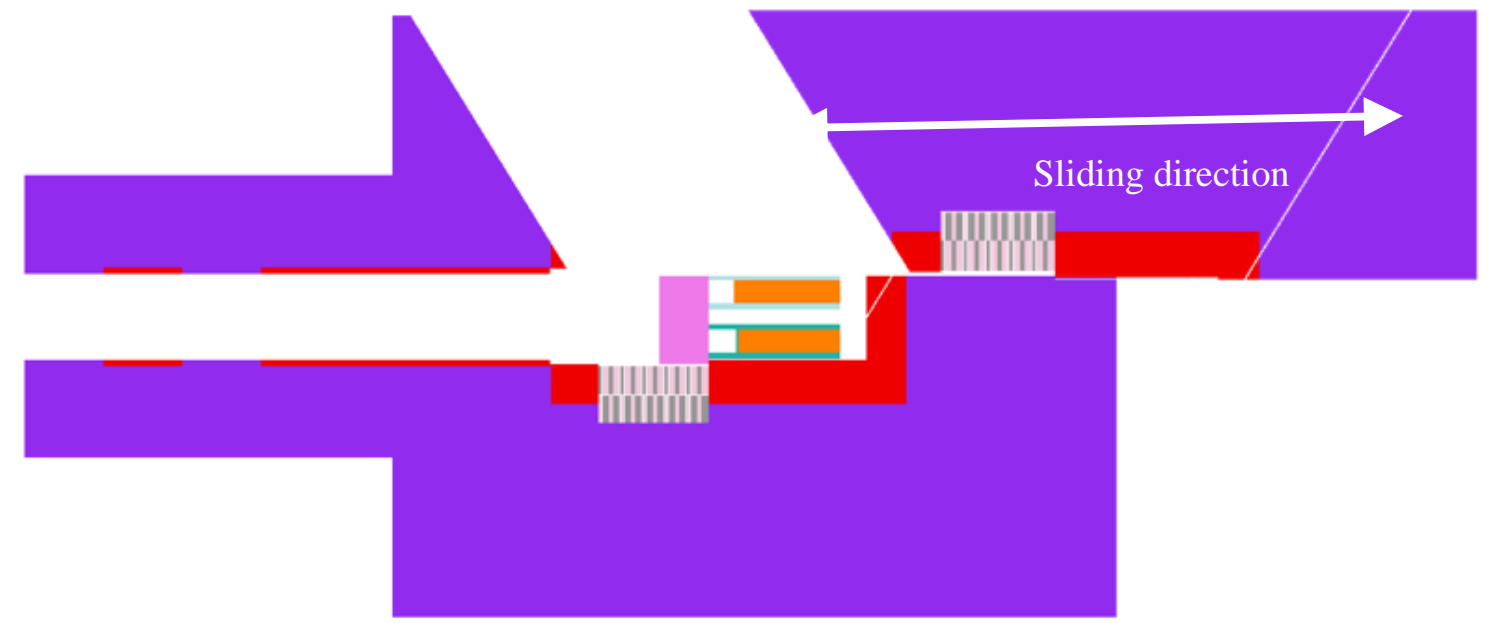

Figure 8. Sliding closure concept (sliding rail and rollers not shown).

The cable channel was modeled as $3 \times 3 \mathrm{~cm}$ in a straight line. However, it is extremely preferable that the cable channel be kinked to avoid a straight shot for radiation entering the shield. One kink in the polyethylene region and one in the lead region is preferable. While kinks should not be smooth, they do not need to have an interior angle of less than 135 degrees, so cables can remain relatively straight. Also, kinks can have increased area or smoothed corners to permit cable bending.

\subsubsection{Polyethylene portion of room return shield}

The mass of borated polyethylene, excluding any polyethylene shielding sample, is $1,350 \mathrm{~kg}$. The diameter used in simulations was $115 \mathrm{~cm}$, but it has been reduced to 110 in the drawing to maintain the mass below 1,400 kg. Reduction in performance can be gauged using graphs provided in the CED-1 report and by using the sensitivity to polyethylene density as shown in the sensitivity study performed for this work.

The polyethylene portion could be constructed by stacking interlocking polyethylene cutouts. This would eliminate the possibilities of streaming paths that are not explicitly part of the design. A 1 in. borated polyethylene sheet is priced at $\$ 1,100$ per $4 \times 8 \mathrm{ft}$. sheet [10]. Assuming $30 \%$ wastage, similar to that in Figure 9, the material cost would be $\$ 31,000$. The cost to fabricate the interlocking parts through computer numerical control (CNC) will also be present. This would allow for fabrication of the opening. Other approaches such as rolling up sheet, casting the BPE, and then cutting require more machining operations that are not as easily controlled. Regardless, the final shape may need to have its bore 
machined to tolerance. A possibility is to include holes for alignment pegs to provide non-adhesive structural support. Based on experience with criticality benchmarks, use of adhesive is highly discouraged.

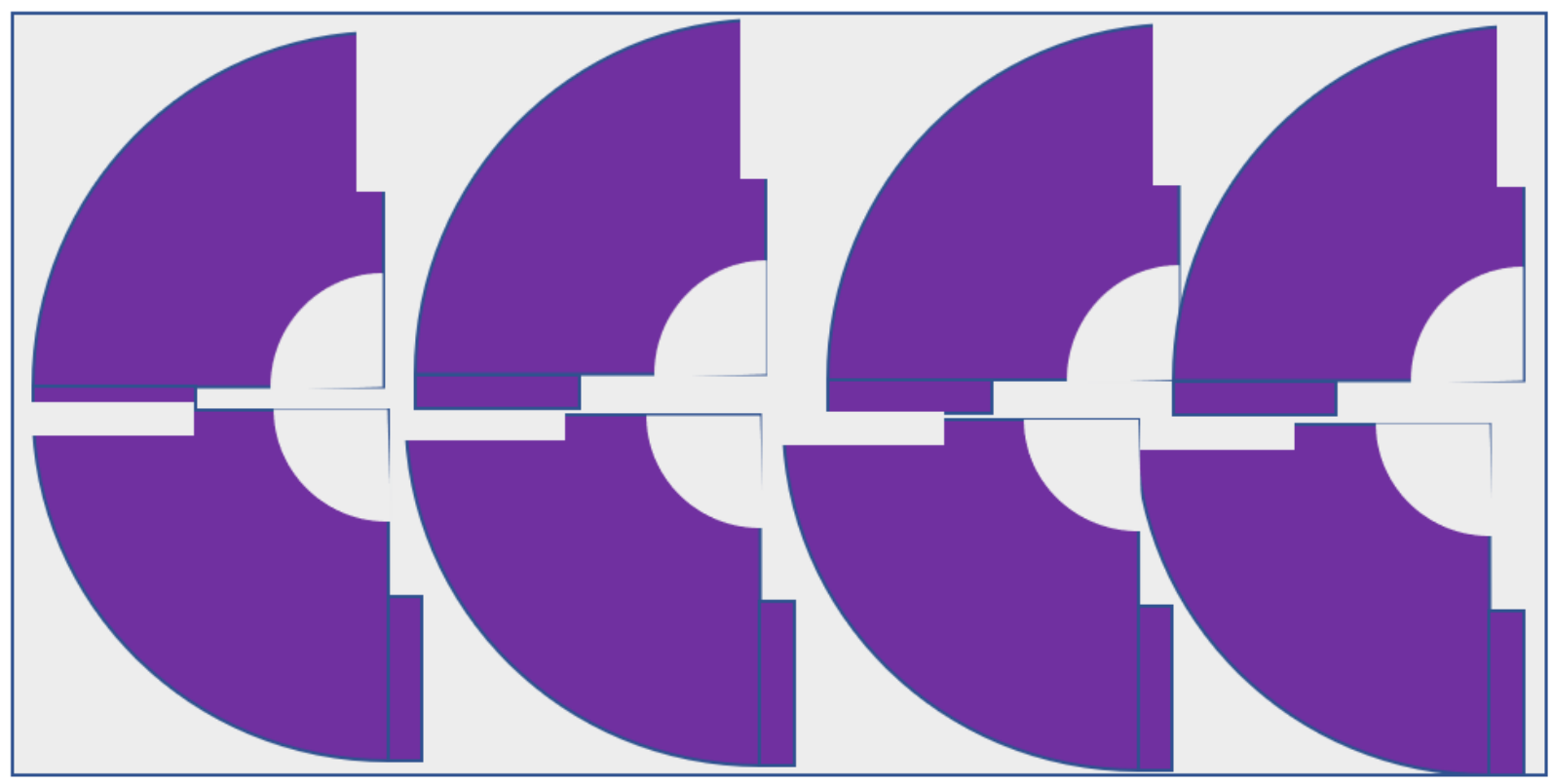

Figure 9. Concept of CNC cutouts of overlapping shield pieces to assemble into room return shield.

A conceptual example of cutouts is shown above. It is likely that two radial layers alternated axially will better prevent streaming. The clamshell opening cut is not shown above.

\subsubsection{Lead portion of room return shield}

The lead portions of the room return shield will require powder coating. Possibilities for construction of the lead portion include assembly from smaller lead parts or lead casting. If lead casting is used, care must be taken to avoid the formation of bubbles and voids during cooling. Either fabrication technique may require machining out the bore and the exterior to tolerances. Lead casting quotes have not been obtained. The price for certified alloy is likely above the scrap price of $\$ 2 / \mathrm{kg}$. With a lead weight of $415 \mathrm{~kg}$, and assuming an alloy price double that of scrap at $\$ 4 / \mathrm{kg}$, the lead material cost is only $\$ 1,600$. Even doubling that value again, for the lead cylinder, fabrication is expected to be the primary cost due to the scale of parts, the custom design, and environmental controls. Note that the cylinder will also open with a sliding mechanism (or similar) for shielding sample and detector placement.

\subsection{SAMPLE CONSTRUCTION}

Shield samples will nominally include one $10 \mathrm{~cm}$ plate, one $5 \mathrm{~cm}$ plate, and $1 \mathrm{~cm}$ plates to allow for a variety of thicknesses to support any diagnostic testing during the experimental campaign. Shield samples should have tight $(<1 \mathrm{~mm})$ clearance with the room return shield to prevent streaming.

\subsection{DETECTORS}

Most of the detectors will be housed within the room return shield cavity. They can be mounted using aluminum sheet metal and brackets. The mounting mechanism geometry and alloy should be recorded for the benchmark. The aluminum should not be between the detectors and the shield sample. A workable 
design is that of round aluminum sheets with holes to hold the detectors. Various designs are acceptable here if the geometry is recorded, the aluminum composition is recorded, the detectors remain in contact with the shield sample, and the aluminum usage is not excessive.

Foils and integral detectors can be mounted on an aluminum plate directly behind the shielding sample. The plate should be no more than $2 \mathrm{~cm}$ from the shield sample, so the detectors can have good communication with the shielding sample. Positions should be fixed to within 1-2 $\mathrm{mm}$ to ensure reproducibility. An example is shown in Figure 10 below. The detectors are placed in rotationally symmetric locations to make the intensities symmetrical. Two groupings of detectors, one with time sensitive detectors and one that can be processed later are proposed. The time-sensitive configuration is shown in Figure 10. Yellow boxes represent LLNL PNAD detectors, green cylinders represent IRSN SNAC detectors, and red circles represent indium foils that can be processed separately at the NCERC count lab. Each of these detectors is discussed later in Section 2.4.2 for passive detectors. The sensitivity study indicates that detector positions within $2 \mathrm{~mm}$ should be acceptable. Positional uncertainty of $1 \mathrm{~mm}$ with respect to the detector chamber walls and the shield sample surface is more ideal.

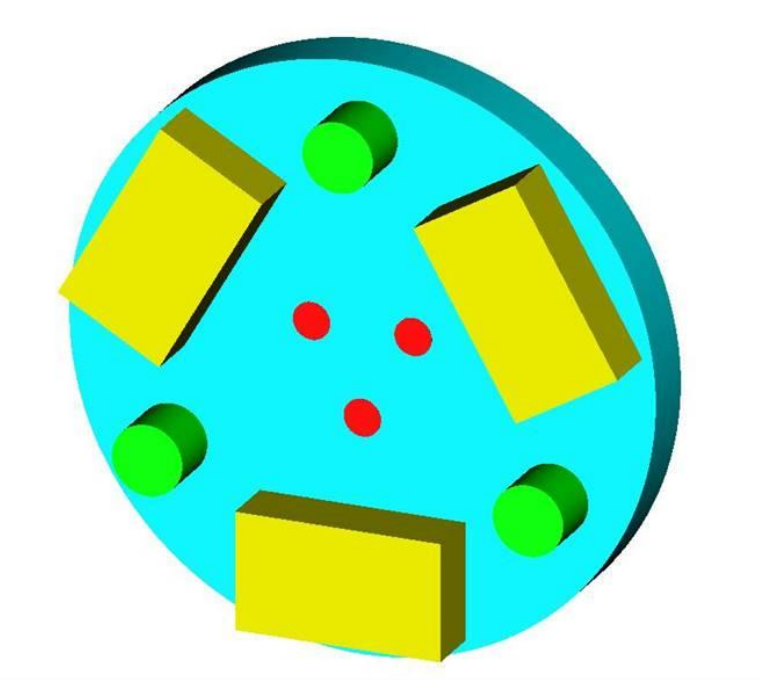

Figure 10. Passive detector mounting arrangement.

\subsubsection{Active detection systems}

Active detection systems are included in the design to inform the benchmarking process. While the final implementation may or may not yield benchmark quality information, the foil data is limited to specific energies, where electronic detectors can capture additional spectral information that may be useful in understanding the function of the test. The detectors may also provide benchmark data in the future depending upon what exactly they measure.

\subsubsection{EJ-309 Fast neutron/gamma organic scintillator detector}

Most responses are expected in the fast portion of the neutron spectrum. A proton recoil scintillator is suitable for measuring neutron spectra in this range. The EJ-309 scintillator is available at NCERC and will fit into the room return shield. EJ-309 uses xylene as a solvent, and it has a higher flashpoint than NE-213/EJ-301, which is a more traditional choice [11].

The detector available at LANL uses pulse shape discrimination (PSD) to identify pulses originating from a gamma or neutron interaction. The system has good PSD for neutrons above $350 \mathrm{keV}$. Notably, detector 
efficiency is decreased below $\sim 1 \mathrm{MeV}$ [12]. Inclusion of a thermal neutron detector and some polyethylene in a second measurement in the same configuration could inform the intermediate/slow portion of the spectrum. Ultimately, the EJ-309 detector shows in detail what is happening in the upper half of the fission spectrum, which is principally uncollided radiation. This should capture the oxygen anti-resonance for $2.36 \mathrm{MeV}$ neutrons, which can stream through unimpeded.

The primary neutron detection mechanism of the EJ-309 scintillator is via protons recoiling from elastic scatter interactions with fast neutrons. Neutrons produce recoil nuclei, which produce differently shaped light pulses than electrons produced by gammas. The electronics attached to the EJ-309 photomultiplier discriminate against gammas by using a short and long charge integration period for a scintillation pulse. This is used to create a shape parameter that can distinguish between neutron pulses and gamma pulses using an onboard field-programmable gate array included with the CAEN 5730 digitizer that will be used. Traditionally, PSD has been performed with high-speed analog electronics.

If possible, the spectrum over a certain threshold energy will be integrated as the primary benchmark metric. Spectrum unfolding capabilities are planned to be implemented by the time IER-498 experiments are carried out, and the most probable energy distribution of the measured neutron spectra will be available and used as benchmark metrics, as well.
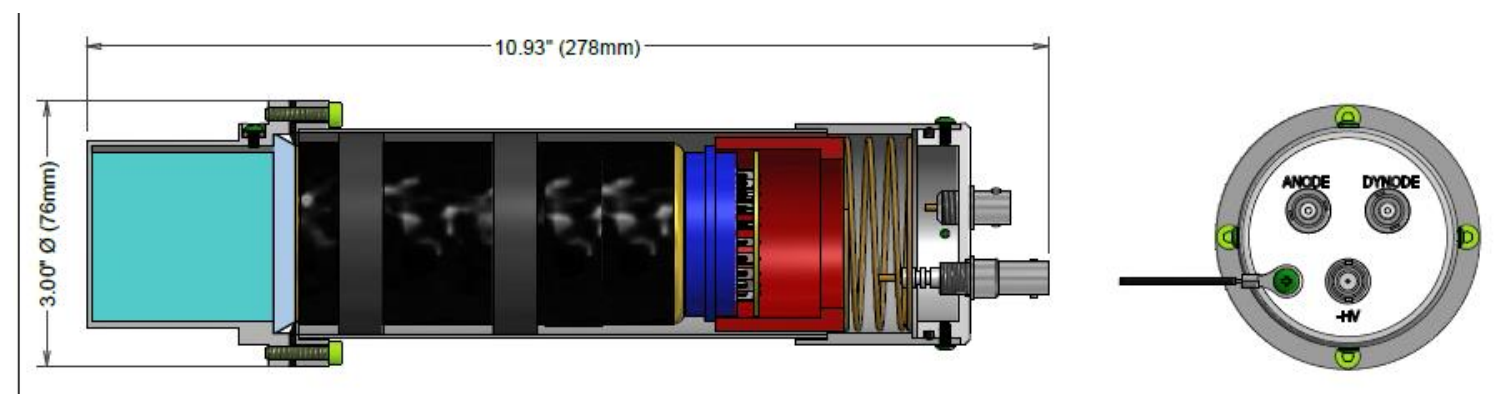

Figure 11. EJ-309 detector [11].

Figure 12 below shows volume-averaged flux tallies of various interactions occurring in the EJ-309 scintillator for a source intensity of 1 watt for 1 second. It was modeled behind a $\mathrm{SiO}_{2}$ shielding sample using the MAVRIC (Monaco with Automated Variance Reduction using Importance Calculations) sequence of Scale 6.2 [13]. This is a Monte Carlo transport calculation with a discrete ordinates calculation to produce importance weightings to reduce variance in the tallies. Reaction tallies represent the number of interactions in a cubic centimeter in a detector for a source intensity of 1 watt for 1 second. If the simulation is accurate, then gammas should not interfere with neutron counts. However, when comparing gamma dose to neutron fluences in simulation to those in IER 147, it was noted that IER 147 had about four times more gammas per neutron than simulated here. Therefore, the PSD may still be essential to block out noise from gammas. The PSD setup can also provide some level of information on the gamma intensity. This can provide some insights into how many neutrons are being counted by the gamma detector presented next. 


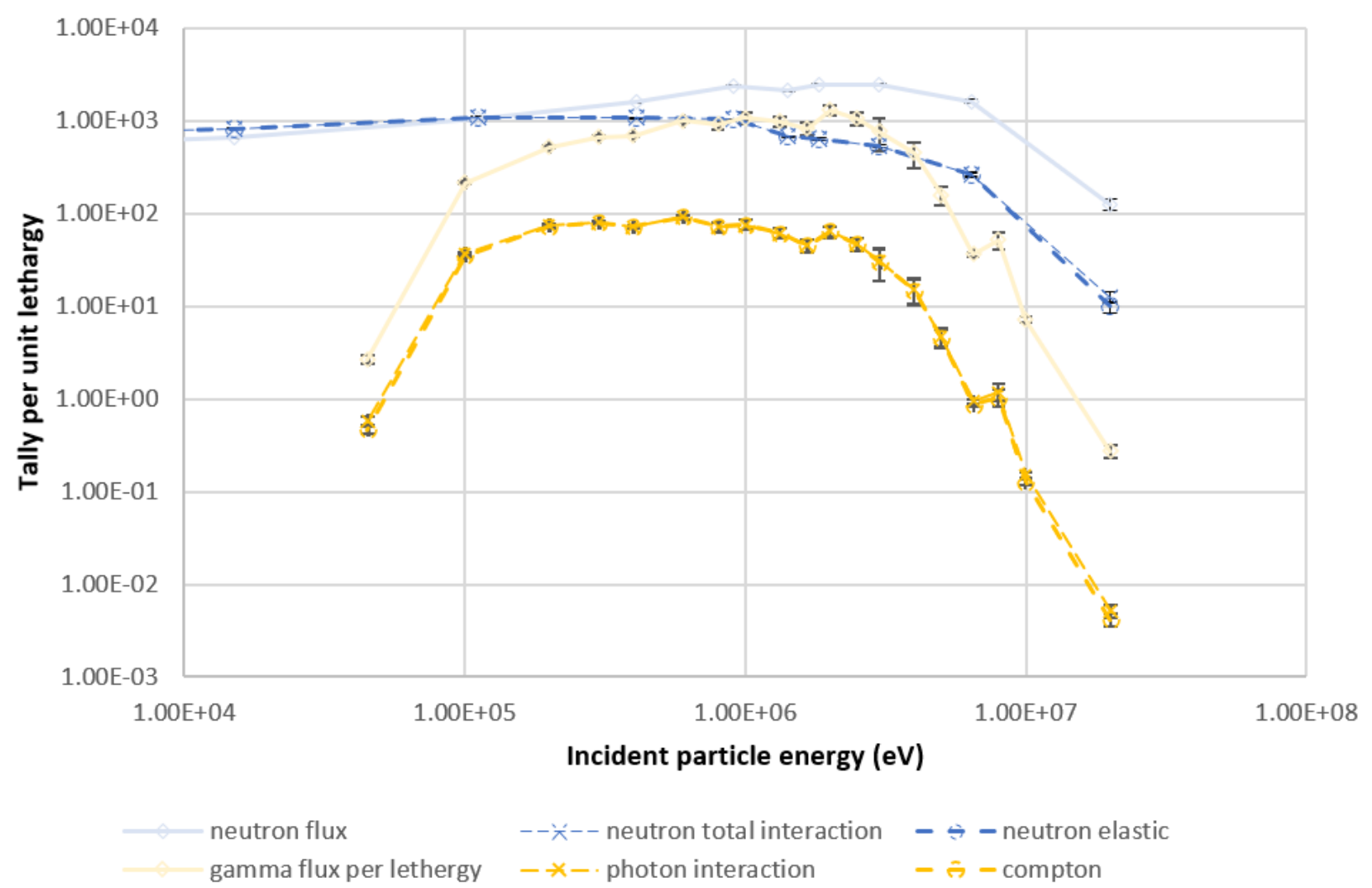

Figure 12. Plot of neutron recoils vs. gamma pulses in the EJ-309 detector (Set000d1_nominal1001).

\subsubsection{SP9 ${ }^{3} \mathrm{He}$ neutron detector}

A 1 in. diameter ${ }^{3} \mathrm{He}$ detector at 2 atmospheres of pressure will count thermal neutrons. This detector was not modeled with the remainder of the simulations. Both are SP9 detectors, each with different gas pressures. The modeled neutron absorption tally is shown below in Figure 13 to demonstrate that gamma interference should not cause a problem. Again, it was modeled behind a $\mathrm{SiO}_{2}$ shielding sample using the MAVRIC sequence of Scale. It is for a 0.2 atmosphere recoil counter, but the 2 atm. tube will have a higher count rate. The 0.2 atm tube will have 12 counts per second at a source intensity of 1 Watt, which is low, so a 2 atm tube seems more appropriate, and should have approximately ten times the count rate, although this is still low compared to the other detectors. The black vertical line is the Q value of $764 \mathrm{keV}$ from the ${ }^{3} \mathrm{He}$ neutron interaction products. It is visible from inspection that the thermal neutron peak is several orders of magnitude larger than the Compton interaction at the $\mathrm{Q}$ value energy, which is good. There are at least 1000 times as many neutron absorptions total as Compton scatters in that energy bin. 


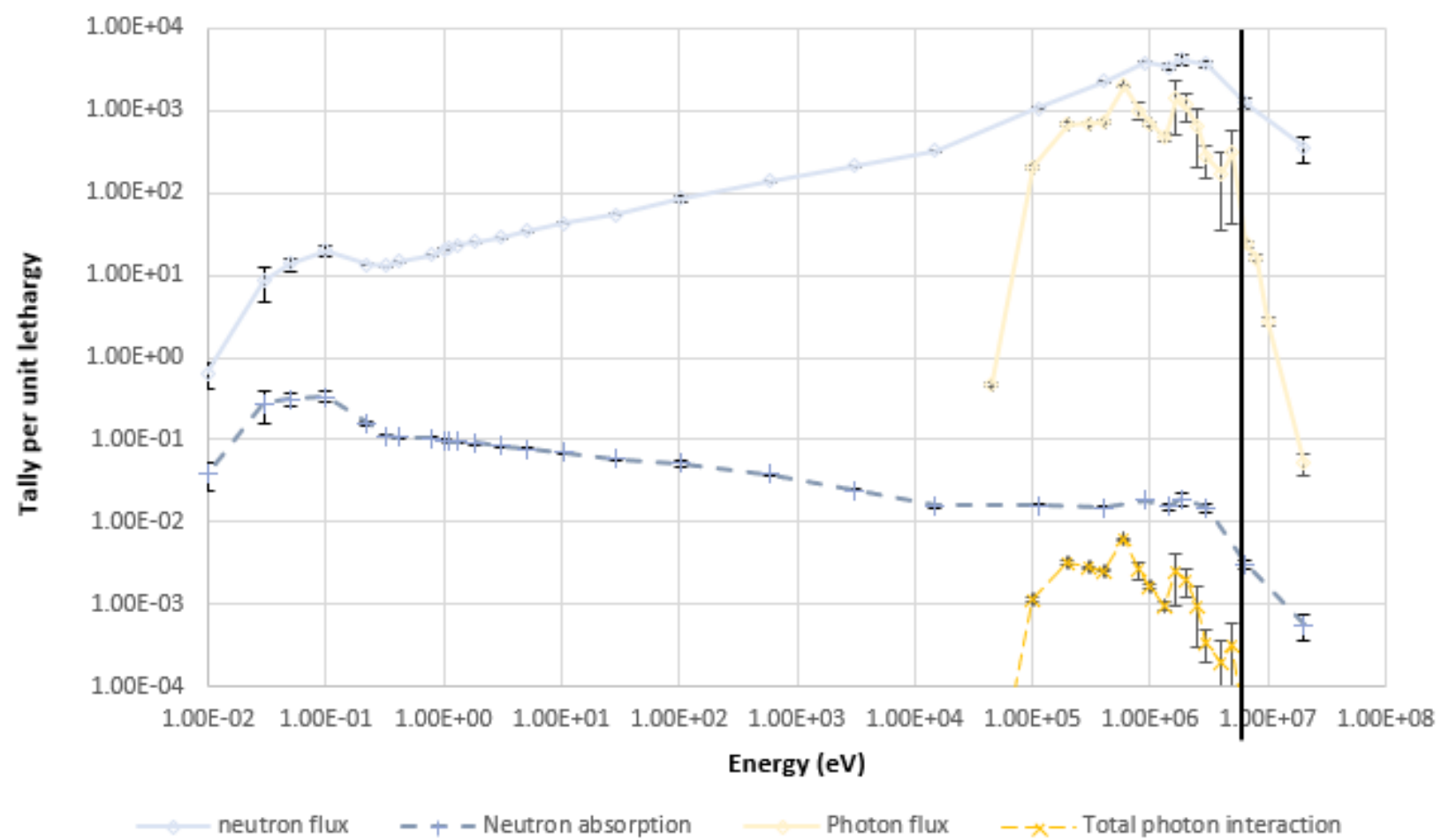

Figure 13. Plot of neutron reactions vs gamma pulses in a $0.2 \mathrm{~atm}$. SP9 detector. The black vertical line is the energy of the neutron reaction with ${ }^{3} \mathrm{He}$.

\subsubsection{Bismuth germanate gamma spectrometer}

A bismuth germanate $\left(\mathrm{Bi}_{4} \mathrm{Ge}_{3} \mathrm{O}_{12}\right.$, or $\left.\mathrm{BGO}\right)$ gamma spectrometer has been selected for the gamma spectrum measurement for delayed critical measurements. Due to the high atomic masses of the BGO constituents, the scintillator receives little energy from fast neutrons. The BGO scintillator proposed here would be a 1-inch right circular cylinder attached to a PMT and Amptek tube base for data transmission to a computer via universal serial bus cable. Recent IRSN evaluations of the BGO approach with ${ }^{137} \mathrm{Cs}$, ${ }^{60} \mathrm{Co}$, and $7 \mathrm{MeV}$ gamma rays against metrological references have shown differences lower than 3\%. BGO does not have an especially sharp spectral response. However, amongst gamma detectors, it has good resistance to neutron flux. Spectral unfolding is performed to obtain a gamma spectrum. An example spectrum using BGO with an AmBe source is shown below in Figure 14 (courtesy of François Trompier at IRSN). 


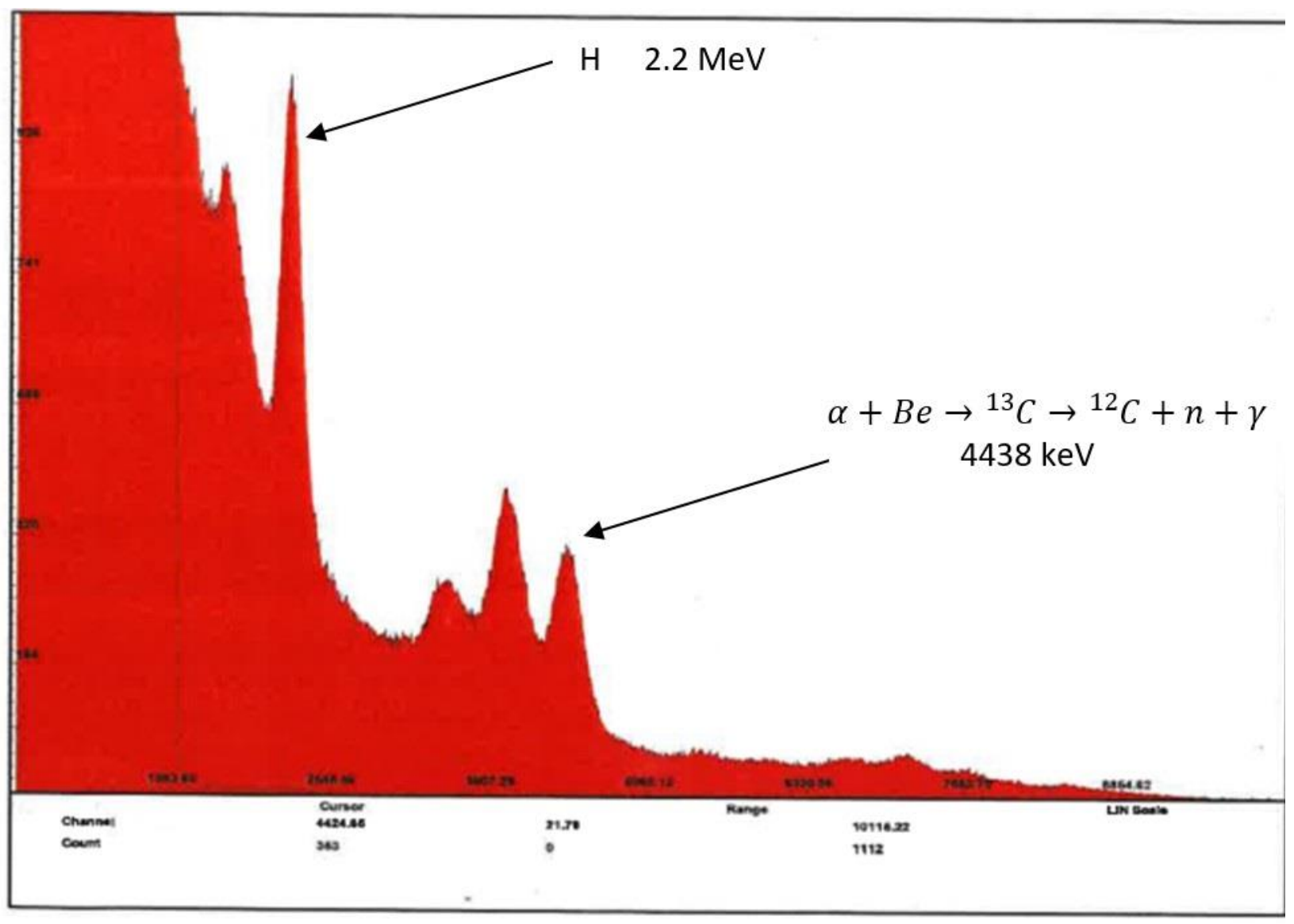

Figure 14. Sample plot of BGO detector response (Courtesy, IRSN).

Energy calibration is typically accomplished with known sources spanning the energy range of interest.

There are numerous options to determine the calculated-to-experimental $(\mathrm{C} / \mathrm{E})$ ratio for gamma measurements. Software such as GADRAS-DRF [14] can in principle reproduce detector responses, but this approach may be complex.

It is most straightforward to identify and examine the height of peaks in the gamma spectrum for comparison between prediction and experiment results. This is especially true if a clearly defined continuum exists for the peak, which can be subtracted off. A complication to this approach is that the shielding sample will change the (an)isotropy of incident radiation, so the escape probability from the scintillator crystal could be an uncontrolled variable in this method. Furthermore, clearly defined peaks may not be present for some samples.

If peaks are not clearly identifiable, then the spectrum can be compared to the prediction using GADRASDRF, combined with simulation results. This approach is more complex and relies on the creation of two simulations. Combining GADRAS-DRF with the simulation also washes out the information on direction traveled by incoming radiation.

Another simple approach is to compare measured total counts above a threshold to prediction, which is the method evaluated here for simplicity. The overall approach of this experiment is to divide some shielded count by an unshielded count, so the detector efficiency should cancel out in this approach. 
However, changes in anisotropy of radiation between the control case and the treatment case would still not be considered.

A fourth option is to compare total counts above a threshold to prediction using simulation and GADRAS-DRF. This approach has similar limitations to using GADRAS-DRF without integrating counts.

A fifth and final option - the feasibility of which is only apparent after measurements are obtained - is to use either GADRAS-DRF or other software to unfold the gamma spectrum. This would remove artifacts such as annihilation peaks and Compton edges to provide some measure of flux.

Figure 15 below shows volume-averaged flux tallies of various interactions occurring in the BGO scintillator for a source intensity of 1 watt with a duration of 1 second. Reaction tallies represent the number of interactions in a cubic centimeter in a detector for the source intensity of 1 watt for 1 second. It appears that most photons of energies over $1 \mathrm{MeV}$ should have only small neutron influence.

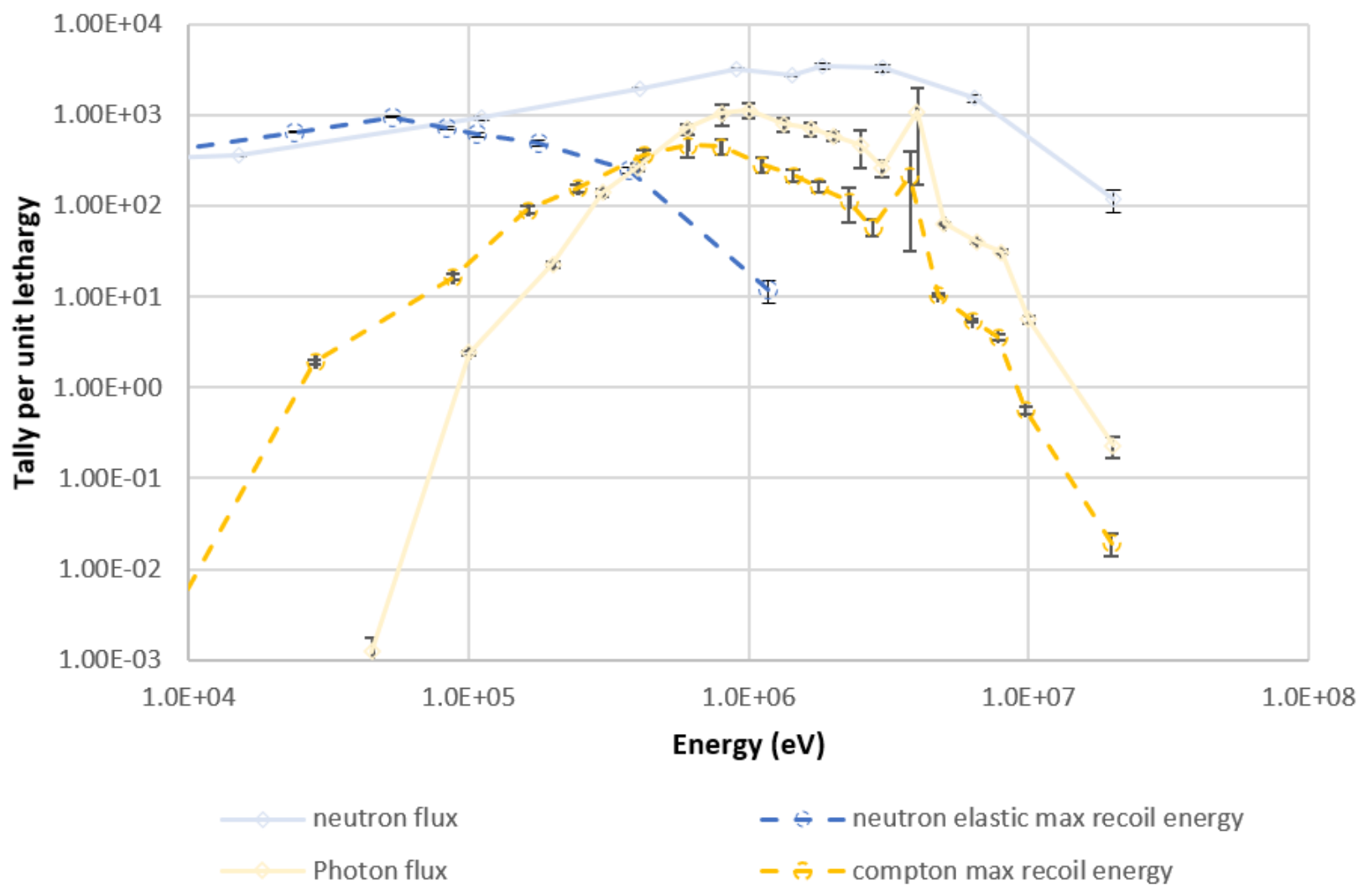

Figure 15. Plot of gammas vs. neutron recoils in BGO crystal. Set000d1_nominal1001.

Possible alternatives include CsI and CYLLC. Dual mode operation of the EJ-309 detector may be the easiest backup.

\subsubsection{Passive detection systems}

Passive detection systems are useful for bursts and high intensity operation where electronic detectors cannot perform as well. They provide less detailed spectral information, but they have uncertainties that 
are easier to evaluate. They do not provide real-time feedback, and some require processing a short time after exposure.

\subsubsection{Neutron activation foils}

For the simulation modeled in this report, LLNL's PNAD was considered as the main technique for neutron dose measurements and neutron spectrum characterization. The LLNL PNAD is composed of a set of neutron activation foils that were specifically chosen for their neutron activation energy range, including a gold foil for thermal neutron, copper for medium energy neutron ( $1 \mathrm{eV}$ to $1 \mathrm{MeV})$, indium for high-energy neutron $(>1 \mathrm{MeV})$, and sulfur for very high-energy neutrons $(>3 \mathrm{MeV})$. A combination of plastic, boron, and cadmium caps/shields are also included in the device. In addition to the foils, a Panasonic TLD is placed in the PNAD. The LLNL PNAD was previously used in different projects with goals similar to that of the IER-498 (e.g., the "Final Design for an International Comparison Exercise for Nuclear Accident Dosimetry at the DAF Using GODIVA-IV [IER-148]" [15] project). The LLNL PNAD concept drawing is shown in Figure 16, and the characteristics of the activation foils and other components are shown in Figure 17. After irradiation, the activated foils must be counted to infer the corresponding neutron flux and spectrum. In the past, LLNL was using Mirion Technologies detectors, including the Falcon HPGe [16] for the gold, copper, and indium foils, and the iSolo [17] for the sulfur foil. The advantage of using those two detectors for foil counting is that they are portable, potentially facilitating the overall experimental setup.

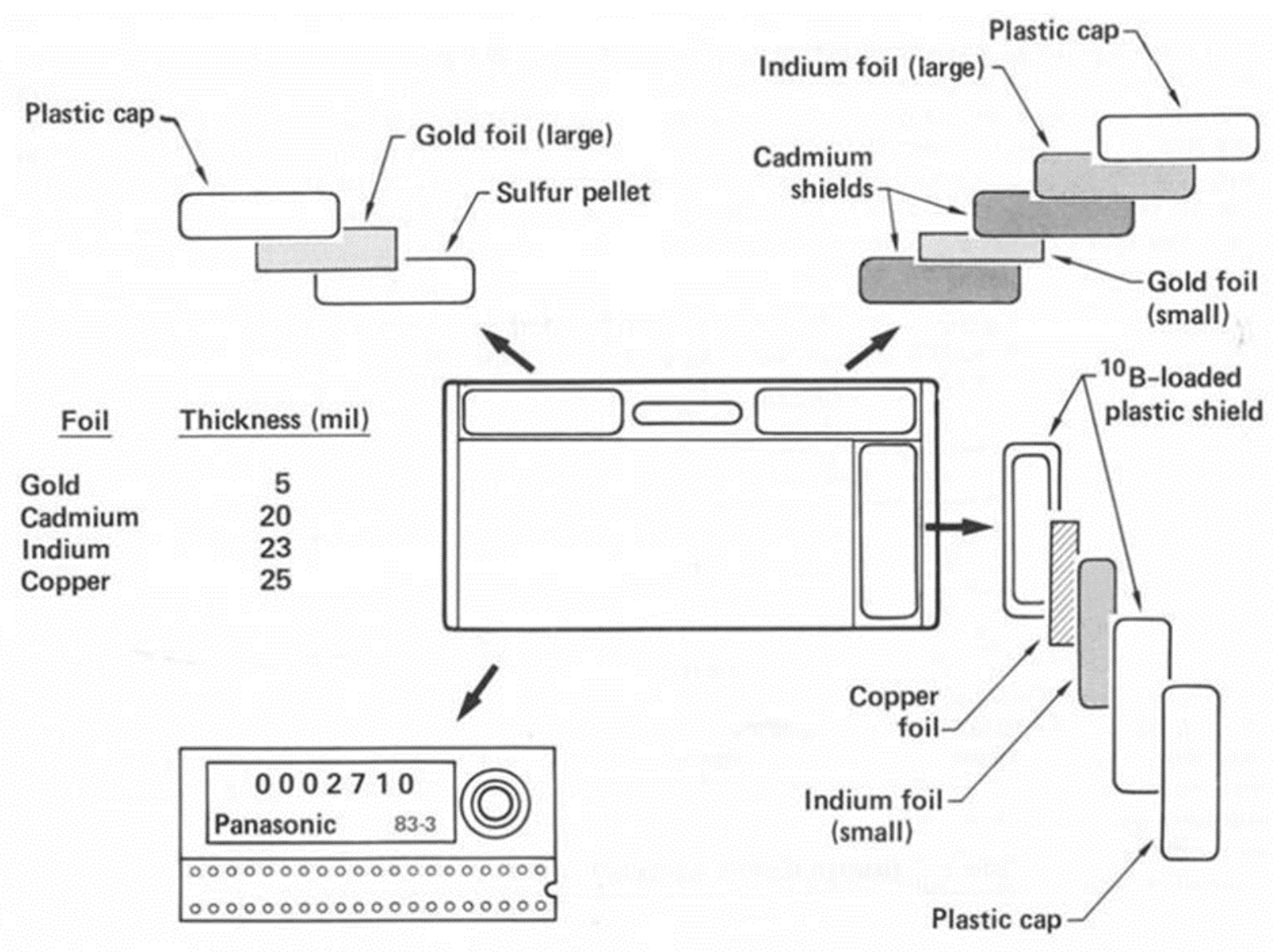

Figure 16. LLNL PNAD main characteristics. 


\begin{tabular}{|l|l|}
\hline Component & Thickness or mass \\
\hline Indium foil & 23 mils \\
Gold foil & 5 mils \\
Cu foil & 25 mils \\
Sulfur pellet & $>0.3$ grams \\
Cadmium & 20 mils \\
Boron & 55 mils middle layer. 60 mils outside layers \\
& ( $80 \%$ by weight. B-10 combined with binder $)$ \\
\hline
\end{tabular}

Figure 17. LLNL PNAD foil and other materials characteristics requirements.

For cross comparison, other potential choices and associations of activation foils and counting detectors are considered. IRSN has developed a similar device, the SNAC2, using a combination of foils for neutron dose measurement and spectrum characterization (see Figure 18). Like the LLNL PNAD, the SNAC2 is composed of different activation foils to cover a broad neutron spectrum, including gold, copper, nickel, indium, magnesium, and ebonite [18]. The SNAC2 has been used in French installations, monitoring for criticality accidents, for over 40 years, notably in Silene and Caliban, and it was also used in IER-148 [15]. After irradiation, the foils can be counted with any gamma spectrometer, for example a NaI scintillator, which is common and portable.

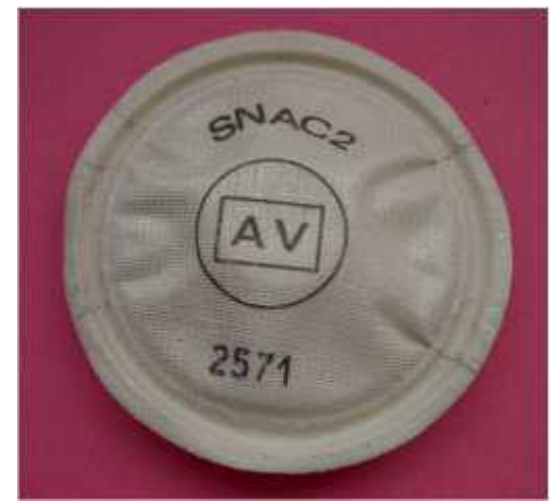

Figure 18. SNAC2 activation neutron spectrometer. (IRSN).

For more cross comparison of Godiva power, dose, and/or neutron spectrum measurements, one approach is to use additional irradiation foils. Shieldwerx sells different sets of neutron activation foils and cadmium covers that are specialized in neutron energy ranges [19]. The purchase of one Fast Neutron Foils Kit SWX-1551 and a Thermal Neutron Foils Kit SWX-1552 would cover all energy ranges and would offer many counting and cross comparison possibilities. The foils in those two sets come in two or four copies and are aluminum, copper, iron, indium, magnesium, nickel, sodium chloride, sulfur, titanium, vanadium, zinc, zirconium, gold, cobalt, $\mathrm{Lu}-\mathrm{Al}, \mathrm{Mn}-\mathrm{Cu}$, molybdenum, scandium, and tungsten; 20 cadmium covers are also supplied. The foils could be counted with one of the detection methods described above.

For both techniques based on the use of activation foils (LLNL and IRSN) and for the individual foils, the optimal application is a high intensity burst, increasing the activation probability and the number of counts registered by the counting detectors and decreasing the measurement uncertainty. Multiple PNADs 
and foils can be placed near Godiva to gain information on the source, and they can also be placed behind the shielding sample to get information on the shielding attenuation. The more devices used to measure the same quantity, the lower the measurement uncertainty will be: hence, the recommendation to use multiple PNADs and multiple foils of the same kind at different locations. As an illustration, one can imagine using 8 PNADs and 8 of each Shieldwerx foil material -4 near Godiva, and 4 behind the shielding sample. Deriving an uncertainty value from activation foils is complex because of the different foil materials and counting methods used. A relative uncertainty of 5 to $10 \%$ can be considered as a maximum value. The sulfur in the LLNL PNAD was measurable down to 40 Bq/g in IER147 [20]. Ideally, a higher activation would be achieved to reduce uncertainty. Several foils are expected to always have near zero counts from the LLNL PNAD when behind a shielding sample, or during low intensity exposure. This is discussed in Section 4 and can be seen inTable 7.

An analysis option for the foils is to use a code such as SAND-IV [21], which can estimate spectra based on foil measurements. While not an exact method, it may be useful.

\subsubsection{Neutron bubble spectrometer}

Bubble Technology Industries produces bubble detectors comprising superheated liquid dispersed in a hydrogel. When a neutron of sufficient energy interacts with the superheated refrigerant, the superheated liquid vaporizes, producing a bubble. The number of bubbles in the detector is proportional to the neutron dose above the threshold energy specific to each detector composition. A set of six detectors in small tubes can be used to characterize a neutron spectrum. These detectors can be placed in the room return shield behind the sample. Their sensitivity would be selected to be the most appropriate for the experiment being performed. To obtain one sample for each energy group, six bubble detectors were modeled as oriented with the broad side toward the sample.

Some detectors are temperature compensated due to the thermodynamic nature of the detection mechanism. However, with age, the temperature compensation mechanisms of detectors in the late 1990s was shown to deteriorate quickly. For the purposes of this work, based on reviewing the work by Vanhavere [22], uncertainty in bubble detector measurement would optimistically be $10 \%$ and would more likely be $20 \%$. With proper care, temperature compensation does not become a large issue.

Dose responses for the various bubble detectors were determined in the 1990s [23]. For the purposes of CED-2, dose response is assumed to follow a threshold stated for the detector in the datasheet.

\subsubsection{Silicon diode as neutron dosimeter}

Silicon diodes offer a very efficient way to measure neutron dose. IRSN diodes are shown in Figure 19. The diodes are small, inexpensive, light, fast, reliable, and potentially reusable. Neutrons interacting with the silicon change its resistivity proportionally to the neutron kerma. Based on a measurement of voltage change through the diode at a constant current, a neutron dose is inferred. The reading is instantaneous, with a simple device such as a multimeter. The IRSN silicon diodes have a neutron detection threshold at $200 \mathrm{keV}$ and a flat energy response up to $10 \mathrm{MeV}$, and they are not sensitive to gammas. Minimal detectable doses are about $50 \mathrm{mGy}$, but 1-5 Gy are optimal. These detectors have also been used previously in IER-148 [15]. The use of multiple silicon diodes in conjunction with the other passive neutron detection systems previously described would provide a simple way to increase confidence in the results. 


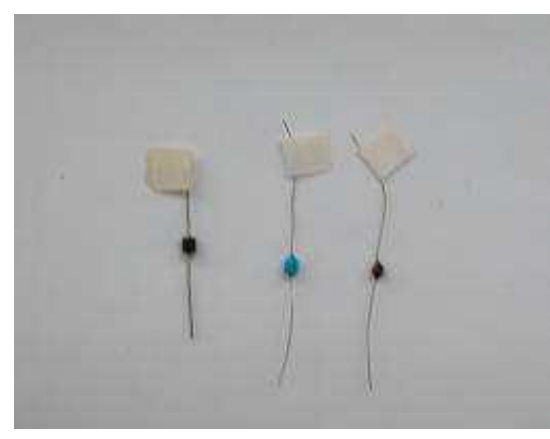

Figure 19. Silicon diodes. (IRSN).

\subsubsection{TLD to measure gamma}

As described above, a Panasonic TLD is available in each of LLNL's PNADs. TLDs are used for gamma dose measurements. LLNL, Sandia and IRSN can also provide $\mathrm{CaF}_{2}$ TLDs, which provide better estimations for gamma doses with minimal neutron influence. A TLD is roughly accurate to $3 \%$ based on a paper for doses at the $0.36 \mathrm{mGy}$ level (calculated as roughly the exposure over the measurement period) [24]. TLDs do not tend to suffer from neutron activation [25]. A report on Sandia Pulsed Reactor (SPR) III, a highly enriched uranium (HEU)-fueled burst assembly with similarities to Godiva IV, notes that activation near the TLD can interfere with the dose measurement [26].

\subsubsection{RPL glass rod and badge to measure gammas}

IRSN can provide the RPL glass rod (GD 351 from Technol) dosimeters shown in Figure 20 and the RPL glass badge dosimeters from the IRSN monitoring service, as shown in Figure 21. RPL glass rods can be read directly onsite with the Dose Ace reader shown in Figure 22 [15]. Both RPL glass rods and badges are energy compensated and have a very low response to neutrons, making them suitable for gamma dose measurement. Dose range is from $50 \mu \mathrm{Gy}$ to $100 \mathrm{~Gy}$ for the rod and $10 \mathrm{~Gy}$ for the badge. RPL dosimeters and TLDs have similar dosimetric properties, but it is always useful to have more cross comparison of measurements from different detection methods.
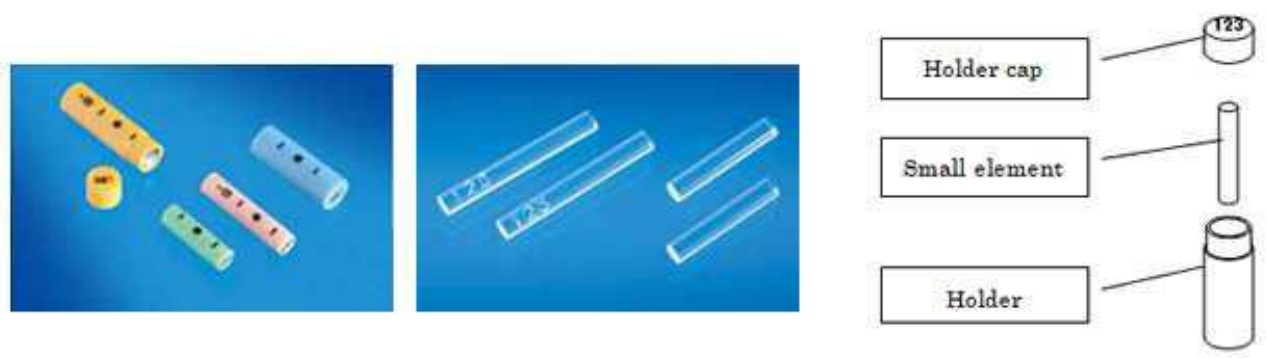

Figure 20. RPL glass rod. 


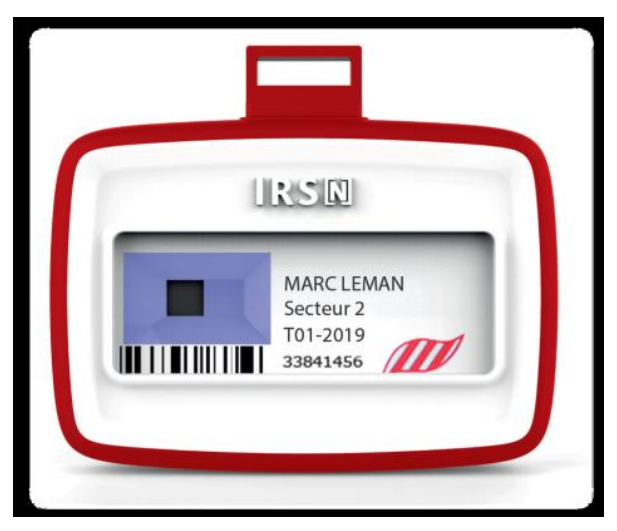

Figure 21. IRSN RPL dosimeter.

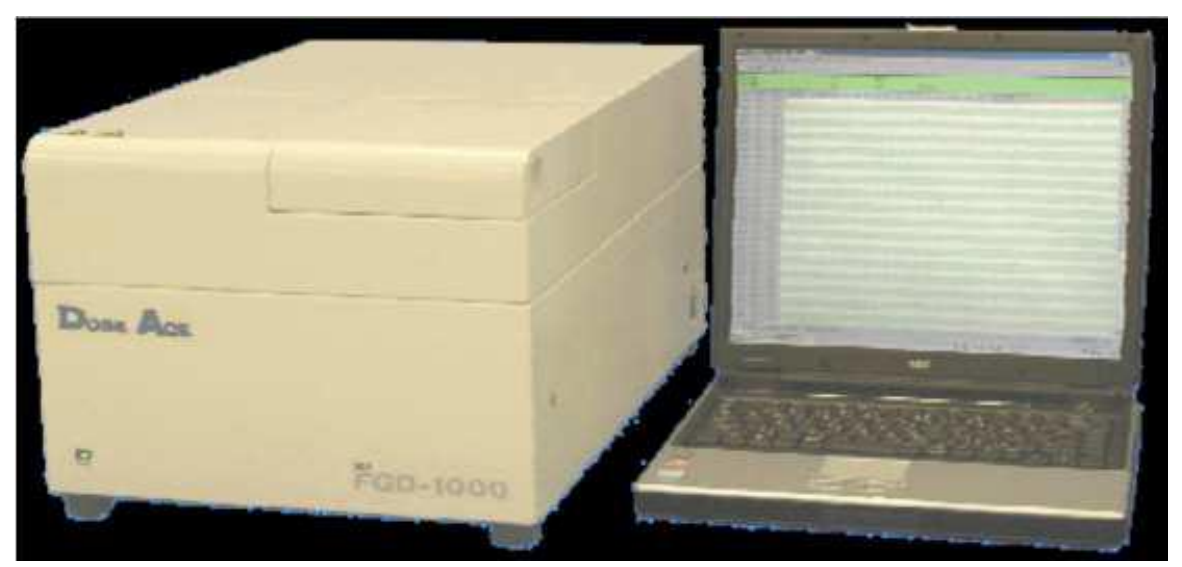

Figure 22. RPL Dose Ace reader for GD 351 glass rod.

\subsubsection{Burst detector mounting arrangement}

For the bursts, detectors are mounted directly to an aluminum mounting plate. The goal is to reduce the aluminum content to be negligible relative to the remainder of the shielding setup and minimize positional uncertainty. For this effort, 1000 series pure aluminum is preferred due to its lack of alloying elements.

\subsubsection{Calibration TLD/foil}

A TLD will be placed near the critical assembly to cross calibrate burst intensities to those of delayed critical intensities. An acceptable location is on the front face of the room return shield. This can facilitate any cross comparisons in CED-4. The feasibility of this approach was demonstrated in the GODIVA-IV vicinity in IER 147. Indium or other foils can also be placed on the front of the room return shield for calibration purposes. This is redundant capability with the range measurement. The foil and TLD are supplemental information because the source instrumentation can also provide this function when combined with the baseline measurement information.

Both the delayed critical and super-prompt critical configurations will have one or more reference measurements made with no shielding sample. Each measurement will be divided by the reference measurement or the average of reference measurements to produce a metric that can be compared to code predictions. Because shielding behaves in an exponential manner, this should remove most lurking variables that are common to both measurements, allowing the effect of the shielding sample and room return shield to be isolated. 


\subsection{DEFINITION OF BENCHMARK QUANTITY}

Numerous passive detector measurements will be made, and each can form the basis for a benchmark. Electronic counts in energy windows could also provide benchmarks, as well as dosimetry measurements. Regardless, to eliminate uncertainty in source intensity, and bias from poorly known source distance, dividing a treatment measurement $\left(C_{\text {Treatment }}\right)$ by a baseline $\left(C_{\text {baseline }}\right)$ is expected to provide a higher quality benchmark quantity than would be produced otherwise. Further, normalizing by some proxy to source strength such as reading of power monitor $(s)$ can compensate for changing source intensities.

If detector or other uncertainty is large, it may make sense to characterize the source well and use counts directly. This is an option, but it is not pursued in this report.

The primary benchmark detector counts will be foil activity and TLD dose because they are easily modeled. Electronic detectors may provide benchmark information but are being included both for diagnostics to ensure understanding of the system. In the case of the foil the benchmark quantity is computed using Eq. (1).

$$
Q=\frac{C_{\text {Treatment }} S_{\text {baseline }}}{C_{\text {baseline }} S_{\text {treatment }}}
$$

A single measurement benchmark consisting of a detector measurement for a given source intensity is more traditional than the two-measurement benchmark defined here. 


\section{FORECASTED MEASUREMENTS AND BENCHMARK QUANTITIES}

The design configuration was modeled with various shielding samples. The system was modeled with Scale 6.2's MAVRIC sequence. The model inputs used in this section model foils and bubble dosimeters, as well as neutron flux estimates behind the sample. Some differences may be encountered with other detectors, but overall, the intensities should remain the same. The room return shield is placed in the model so that the center of the back side of the shield sample is assumed to be $3.66 \mathrm{~m}$ from the center of the Godiva-IV assembly, close enough to get good intensity, but far enough to avoid interfering with assembly operation.

\subsection{NEUTRON FLUX}

Figure 23 below shows fluxes as a fraction of the baseline measurement with no shielding sample in the room return shield. In the figure, each energy group is divided by the intensity of the $1.3 \mathrm{~cm}$ thick lead sample, and that value is plotted. For all materials except for PE, the changes are restricted to the fast part of the spectrum above approximately $500 \mathrm{keV}$, where the recoil detector is sensitive. The intensity below $500 \mathrm{keV}$ significantly changes with energy only for the PE cases. These are flux ratios and not fluxes.

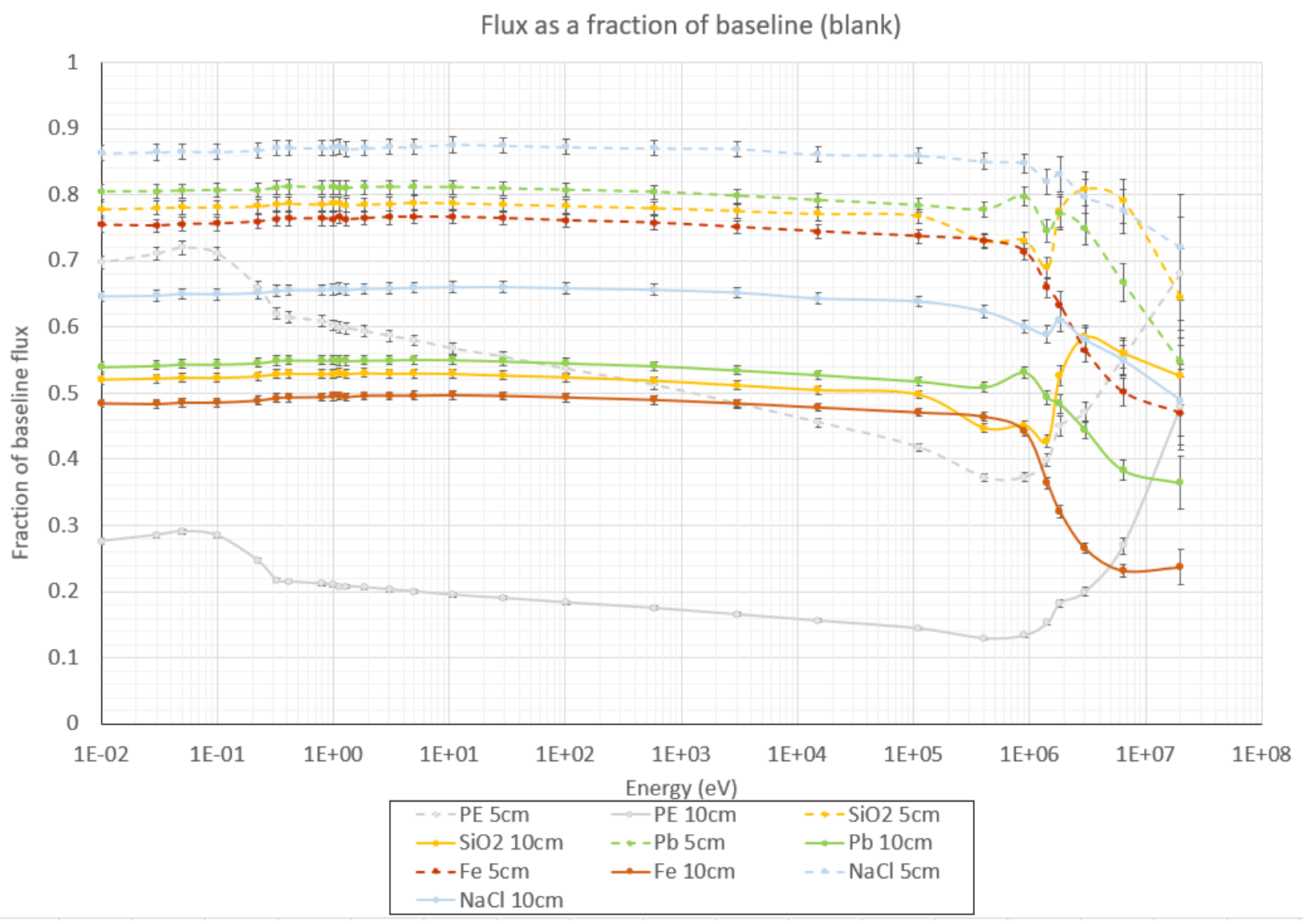

Figure 23. Plot of flux as a fraction of baseline for various shielding materials.

The benchmark quantity will be the natural logarithm of the intensity above an energy threshold divided by the same measurement for a reference case. Figure 24 shows the fluxes used to make Figure 23. 
Because these tallies are taken from a bubble dosimeter, there is an increased thermal peak compared to what is seen in a gas detector such as the ${ }^{3} \mathrm{He}$ SP9 detector.

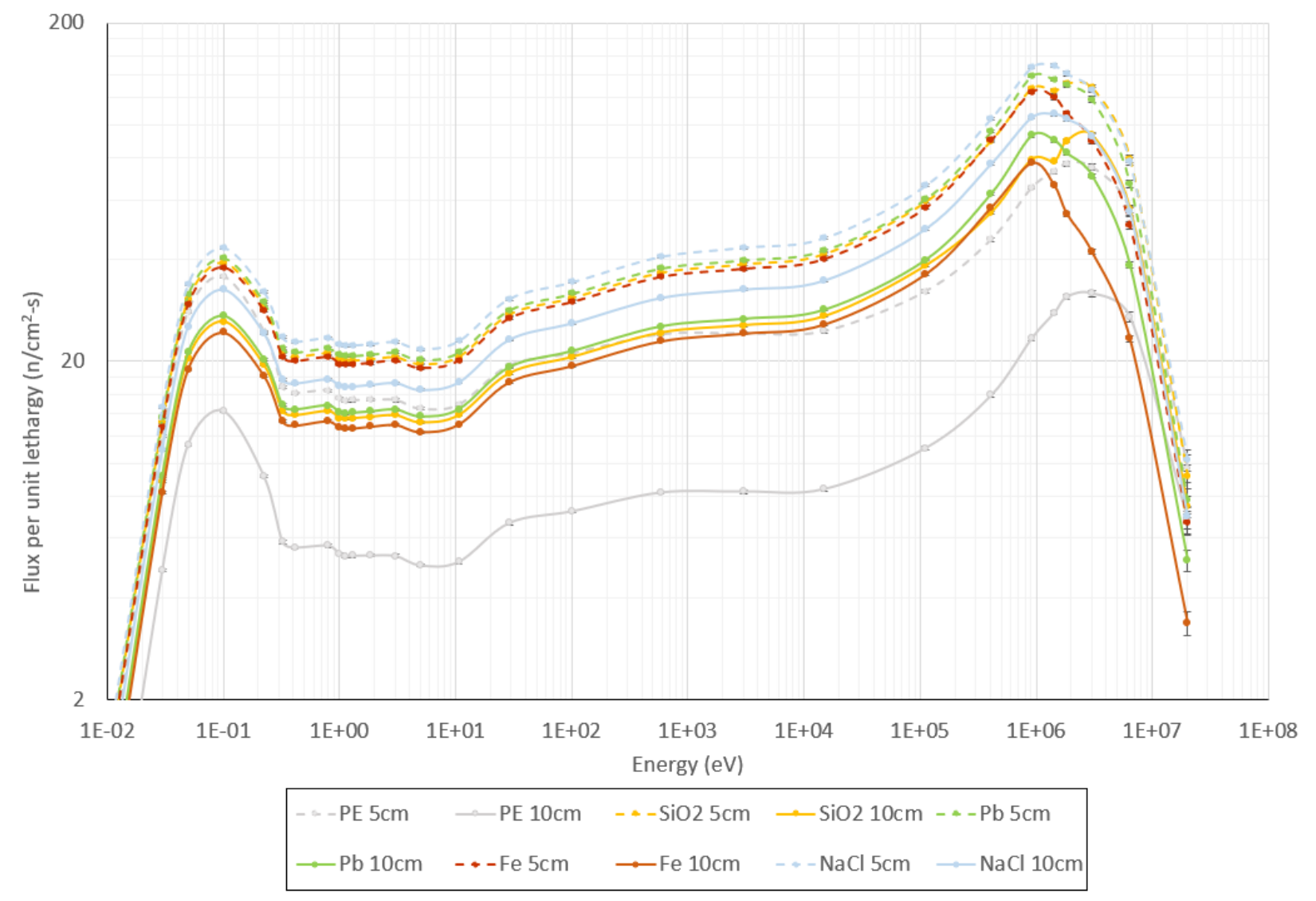

Figure 24. Flux in bubble spectrometer detectors.

\subsection{GAMMA FLUX}

Prediction of where specific peaks are located is difficult using the current models. Identifying and tracking peak measurements may be useful for benchmarking or for verifying that the experiment behaves as expected. Gamma attenuation coefficients are rather smooth with respect to energy for energies above approximately $100 \mathrm{keV}$, so the peaks would be from secondary gamma production or gamma production at the source.

Model convergence for gammas was slow, so detailed spectra were not modeled. Regardless, lower fidelity models provide some baseline expectation.

Gamma intensity through a polyethylene shield sample exceeds the baseline gamma measurements at several energies, as seen below in Figure 25. There appears that there may be a peak in gammas when using a polyethylene shielding sample around $2 \mathrm{MeV}$. Capture cross sections for fast neutrons in the fission spectrum range tend to be small, so it is reasonable to expect that thermalization in the polyethylene enables more secondary gamma production. The tallies were simulated as being taken in a square patch of $\mathrm{CaF}_{2}$. 


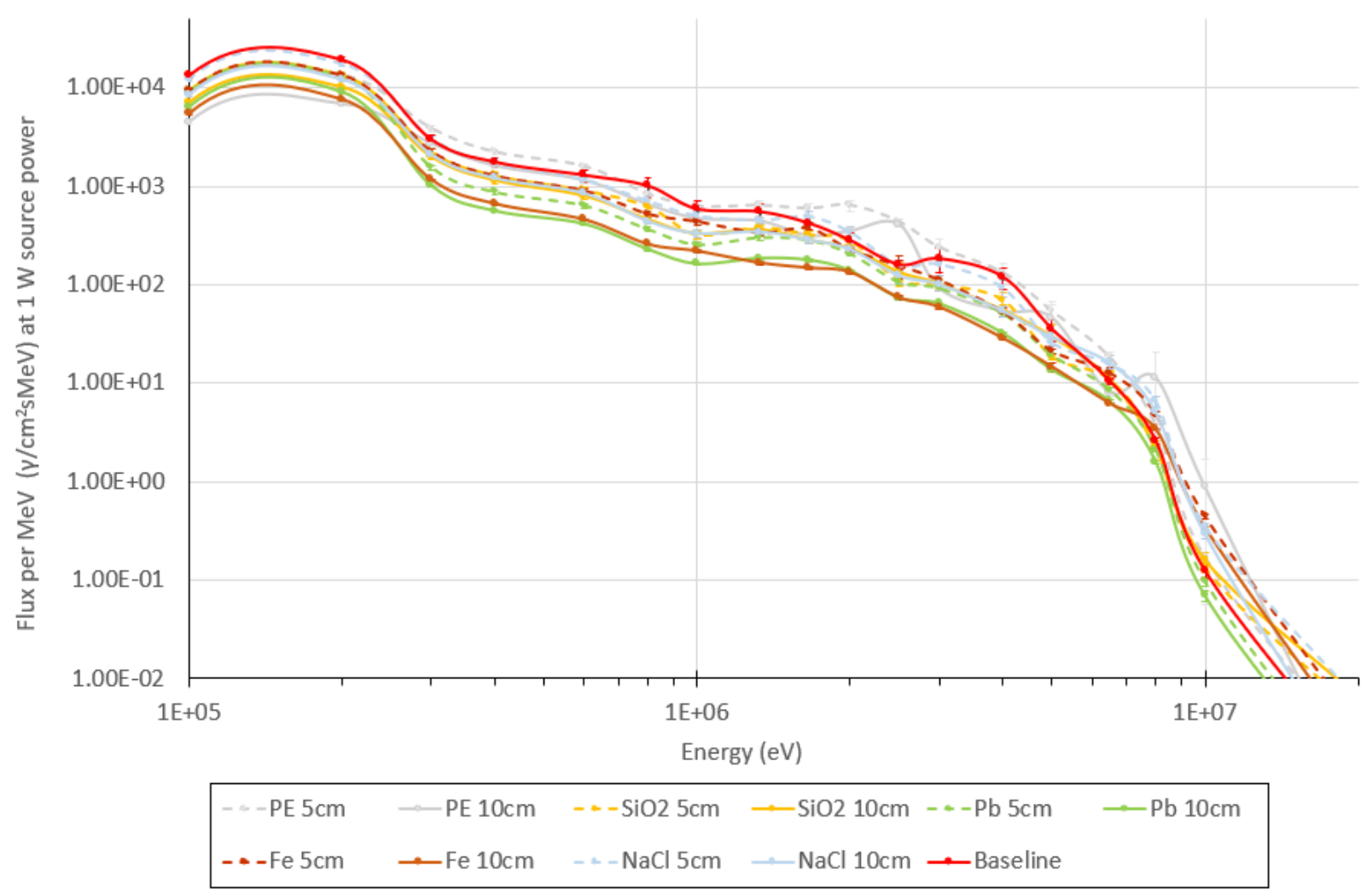

Figure 25. Simulated gamma spectra for various samples. Higher resolution would likely reveal more peaks.

A different view is seen in Figure 26 below by dividing the gamma tally by the baseline (no shield material in front of detector) value. A peak in the polyethylene is seen at around $2 \mathrm{MeV}$. The increases in gamma intensity versus baseline at energies above approximately $8 \mathrm{MeV}$ could be from various secondary gamma production reactions. Iron samples appear to peak in the energy group with the $10 \mathrm{MeV}$ upper limit, as does $\mathrm{NaCl}$. Lead does not appear to have any appreciable differences versus baseline. This could be because lead is a strong gamma absorber or because the area is already saturated with lead absorption gammas from the room return shield. 


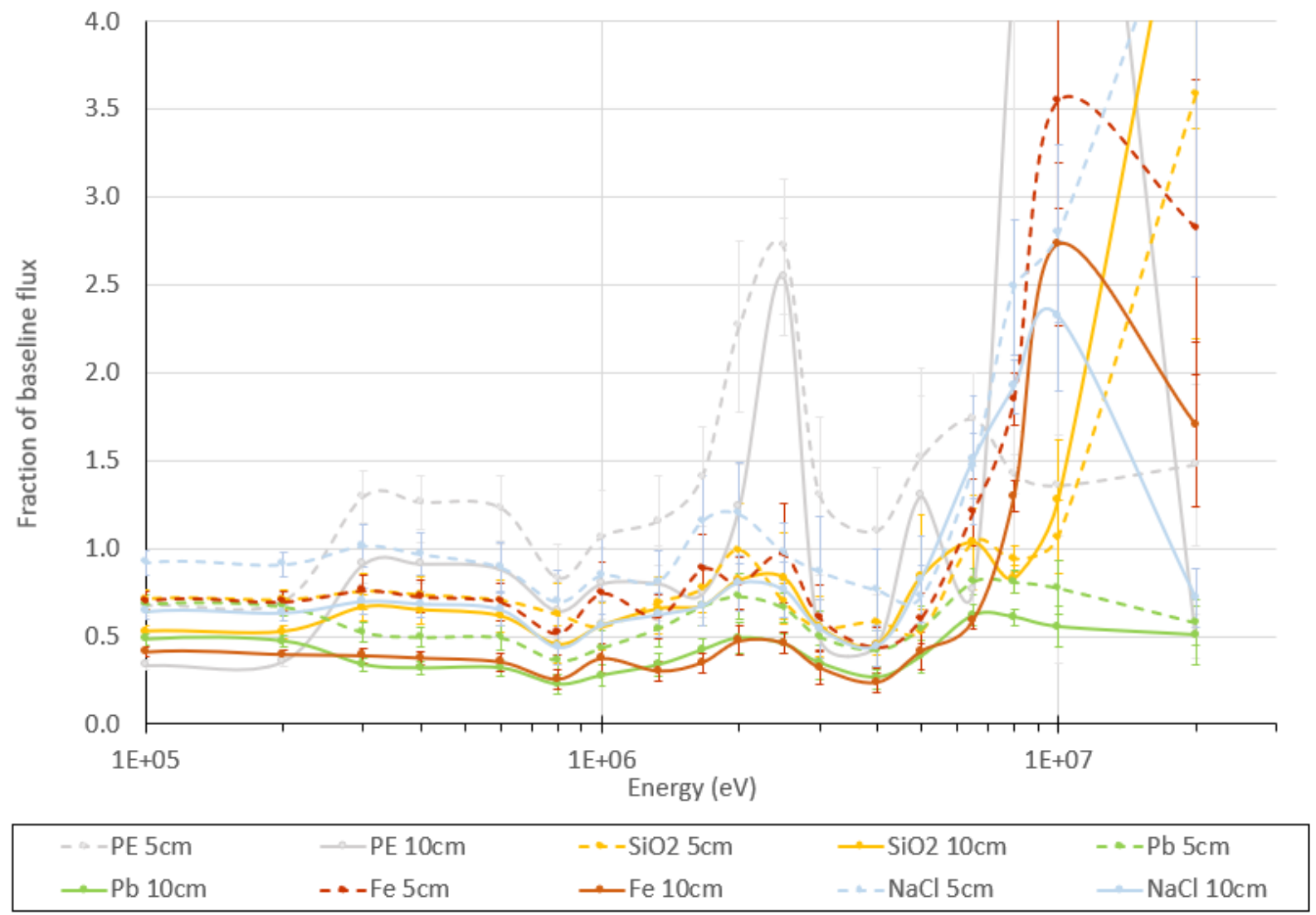

Figure 26. Gamma fluxes as a fraction of gamma fluxes with no shielding samples.

\subsection{PNAD FOIL ACTIVITIES}

The PNAD [15] was modeled. The various foils responses were used to determine activities at two intensities and exposure times. This informs the next section on source intensities as necessary for good measurements. 
Table 1. Foil activities (Bq) for $10 \mathrm{~W}$ source power for 4,000 seconds with three hours of decay

\begin{tabular}{|c|c|c|c|c|c|c|c|}
\hline \multirow[b]{2}{*}{$\begin{array}{c}\text { Shielding } \\
\text { material }\end{array}$} & \multicolumn{7}{|c|}{ Activity $(B q)$} \\
\hline & Gold & Sulfur & $\begin{array}{c}\text { Indium } \\
116 \mathrm{~m}\end{array}$ & $\begin{array}{l}\text { Gold (Cd } \\
\text { shielded) }\end{array}$ & $\begin{array}{c}\text { Indium } \\
\text { 116m (B10 } \\
\text { shielded) }\end{array}$ & $\begin{array}{c}\text { Copper } \\
\text { (B10 } \\
\text { shielded) }\end{array}$ & $\begin{array}{c}\text { Indium } \\
115 \mathrm{~m}\end{array}$ \\
\hline None & 4.5 & 0.3 & 91.7 & 4.1 & 26.8 & 2.5 & 47.9 \\
\hline$C 10 \mathrm{~cm}$ & 5.9 & 0.3 & 65.4 & 4.2 & 30.3 & 3.0 & 50.1 \\
\hline$C 5 \mathrm{~cm}$ & 5.7 & 0.3 & 71.9 & 4.3 & 28.1 & 2.6 & 46.7 \\
\hline$F e 10 \mathrm{~cm}$ & 4.5 & 0.1 & 55.6 & 4.1 & 22.9 & 2.1 & 16.4 \\
\hline$F e 5 \mathrm{~cm}$ & 5.3 & 0.2 & 64.9 & 4.6 & 29.6 & 2.9 & 31.0 \\
\hline $\mathrm{NaCl} 10 \mathrm{~cm}$ & 4.5 & 0.2 & 54.2 & 4.3 & 25.2 & 2.8 & 31.8 \\
\hline $\mathrm{NaCl} 5 \mathrm{~cm}$ & 5.0 & 0.2 & 58.6 & 4.3 & 28.9 & 3.1 & 37.6 \\
\hline$P b 10 \mathrm{~cm}$ & 6.0 & 0.1 & 72.7 & 5.1 & 25.1 & 2.3 & 24.3 \\
\hline$P b 5 \mathrm{~cm}$ & 6.1 & 0.2 & 76.8 & 4.9 & 31.2 & 3.3 & 35.7 \\
\hline$P E 10 \mathrm{~cm}$ & 31.0 & 0.1 & 428.6 & 5.9 & 57.2 & 4.2 & 11.0 \\
\hline$P E 5 \mathrm{~cm}$ & 60.8 & 0.1 & 861.9 & 16.9 & 125.4 & 9.2 & 22.1 \\
\hline $\mathrm{SiO}_{2} 10 \mathrm{~cm}$ & 6.6 & 0.2 & 84.0 & 5.7 & 27.3 & 2.5 & 29.3 \\
\hline $\mathrm{SiO}_{2} 5 \mathrm{~cm}$ & 6.8 & 0.2 & 93.9 & 5.3 & 32.4 & 3.0 & 49.2 \\
\hline
\end{tabular}

Table 2. Foil activities (Bq) for $70^{\circ} \mathrm{C}$ burst after 3 hours of decay

\begin{tabular}{|c|c|c|c|c|c|c|c|}
\hline \multirow[b]{2}{*}{$\begin{array}{l}\text { Shielding } \\
\text { material }\end{array}$} & \multicolumn{7}{|c|}{ Activity $(B q)$} \\
\hline & Gold & Sulfur & $\begin{array}{c}\text { Indium } \\
116 \mathrm{~m}\end{array}$ & $\begin{array}{c}\text { Gold } \\
\text { (Cd shielded) }\end{array}$ & $\begin{array}{l}\text { Indium 116m } \\
\text { (B10 shielded) }\end{array}$ & $\begin{array}{c}\text { Copper } \\
\text { (B10 } \\
\text { shielded) }\end{array}$ & $\begin{array}{c}\text { Indium } \\
115 \mathrm{~m}\end{array}$ \\
\hline None & 62.1 & 3.6 & 1858.0 & 55.8 & 542.6 & 35.1 & 711.2 \\
\hline$C 10 \mathrm{~cm}$ & 81.5 & 4.3 & 1325.1 & 57.6 & 614.3 & 41.9 & 743.9 \\
\hline C $5 \mathrm{~cm}$ & 78.4 & 4.3 & 1457.4 & 59.5 & 568.9 & 36.5 & 693.9 \\
\hline$F e 10 \mathrm{~cm}$ & 62.4 & 0.9 & 1126.1 & 56.1 & 464.8 & 29.8 & 244.0 \\
\hline Fe $5 \mathrm{~cm}$ & 72.1 & 2.4 & 1316.0 & 63.6 & 600.5 & 40.6 & 461.0 \\
\hline $\mathrm{NaCl} 10 \mathrm{~cm}$ & 62.3 & 2.2 & 1098.7 & 58.4 & 510.8 & 39.2 & 471.9 \\
\hline $\mathrm{NaCl} 5 \mathrm{~cm}$ & 68.5 & 2.8 & 1187.2 & 59.3 & 586.6 & 43.7 & 559.1 \\
\hline $\mathrm{Pb} 10 \mathrm{~cm}$ & 81.8 & 1.4 & 1473.5 & 70.5 & 509.3 & 33.0 & 360.6 \\
\hline$P b 5 \mathrm{~cm}$ & 84.1 & 2.8 & 1556.1 & 67.2 & 632.8 & 46.4 & 530.8 \\
\hline$P E 10 \mathrm{~cm}$ & 426.3 & 1.0 & 8687.9 & 81.3 & 1159.9 & 59.4 & 162.9 \\
\hline$P E 5 \mathrm{~cm}$ & 835.6 & 1.9 & 17471.7 & 231.9 & 2541.1 & 129.3 & 327.7 \\
\hline $\mathrm{SiO}_{2} 10 \mathrm{~cm}$ & 90.0 & 2.1 & 1703.0 & 77.9 & 553.7 & 35.1 & 435.7 \\
\hline $\mathrm{SiO}_{2} 5 \mathrm{~cm}$ & 93.8 & 2.9 & 1903.2 & 72.7 & 656.1 & 42.2 & 731.0 \\
\hline
\end{tabular}

\subsection{BUBBLE SPECTROMETER}

Table 3 shows the calculated dose at each location for neutrons having energies at thresholds corresponding to the nominal thresholds of the bubble spectrometer. A threshold of $0 \mathrm{eV}$ is also shown to illustrate the contribution of thermal neutrons to the dose not recorded by bubble formation. Uncertainties are simply $10 \%$ of the computed dose, which may be an underestimate. Using a nominal value of 0.15 
bubbles per $\mu \mathrm{Sv}$ as per manufacturer specification [27], and a power of $1 \mathrm{~W}$. The bubble production rate is computed in the table, as well as the time needed to produce 100 bubbles (the maximum that is easily countable). Finally, the number of bubbles in each detector is computed for the lowest count time, along with the percentage of each measurement corresponding to a single bubble.

Table 4 shows a possible use case for the bubble spectrometer. It also shows the differences in bubble counts between detectors with adjacent thresholds. A count uncertainty is shown, as well as an overall measure of uncertainty based on an assumed detector uncertainty of 5\%, which is optimistic [23]. A further assumption is that the most sensitive spectrometer would be exposed until 100 bubbles form because the quantity of 100 bubbles represents what can be reliably counted without excessive overlap. Then the remaining detectors would have a lesser number of bubbles because less dose is imparted at the lower energies for the threshold detectors.

A possible means to lower uncertainty is to use as many bubble detectors as can fit behind the shielding sample. This would allow more bubbles to be generated. Locations of each detector would need to be recorded, and they would ideally be changed from test to test to avoid covariance between detector position and energy group. However, achieving optimal exposure could require one burst per measurement point, which is not ideal. This is seen better in Section 4.

Table 3. Neutron dose for various energy thresholds, bubble production rates, and number of bubbles at 25 minutes

\begin{tabular}{ccccccc}
\hline $\begin{array}{c}\text { Energy } \\
\text { Threshold } \\
(\mathbf{e V})\end{array}$ & $\begin{array}{c}\mathbf{5} \mathbf{~ c m ~ P E} \\
\text { neutron dose } \\
(\boldsymbol{\mu S v} / \mathbf{W}-\mathbf{s})\end{array}$ & $\begin{array}{c}\text { PE } \mathbf{5} \text { cm } \\
\text { uncertainty } \\
(\boldsymbol{\mu} \mathbf{S} \text { /W-s })\end{array}$ & $\begin{array}{c}\text { Bubbles/min @ } \\
\mathbf{1 W}\end{array}$ & $\begin{array}{c}\text { Time to 100 } \\
\text { bubbles (min) }\end{array}$ & $\begin{array}{c}\text { Bubbles @ 25 } \\
\text { min }\end{array}$ & $\begin{array}{c}\text { \% of } \\
\text { measurement } \\
\text { per bubble }\end{array}$ \\
\hline $\mathbf{0 . 0 0 E + 0 0}$ & 0.46 & 0.05 & 4.15 & 24.11 & & \\
$\mathbf{1 . 0 0 E + 0 4}$ & 0.44 & 0.04 & 4.00 & 24.98 & 100.00 & $1 \%$ \\
$\mathbf{1 . 0 0 E + 0 5}$ & 0.44 & 0.04 & 3.99 & 25.09 & 99.54 & $1 \%$ \\
$\mathbf{6 . 0 0 E + 0 5}$ & 0.39 & 0.04 & 3.54 & 28.24 & 88.45 & $1 \%$ \\
$\mathbf{1 . 0 0 E + 0 6}$ & 0.31 & 0.03 & 2.80 & 35.77 & 69.83 & $1 \%$ \\
$\mathbf{2 . 5 0 E + 0 6}$ & 0.20 & 0.02 & 1.80 & 55.44 & 45.05 & $2 \%$ \\
$\mathbf{1 . 0 0 E + 0 7}$ & 0.03 & 0.00 & 0.24 & 414.21 & 6.03 & $17 \%$ \\
\hline
\end{tabular}

Table 4. Uncertainties for exposure of all bubble detectors for $25 \mathrm{~min}$

\begin{tabular}{ccccc}
\hline $\begin{array}{c}\text { Lower energy } \\
(\mathbf{e V})\end{array}$ & $\begin{array}{c}\text { Upper energy } \\
(\mathbf{e V})\end{array}$ & $\begin{array}{c}\text { Difference in } \\
\text { bubbles @ 25.0 } \\
\text { min }\end{array}$ & $\begin{array}{c}\text { Uncertainty } \\
\text { assuming } 1 / 2 \\
\text { bubble count } \\
\text { uncertainty }\end{array}$ & $\begin{array}{c}\text { Uncertainty } \\
\text { assuming 5\% } \\
\text { uncertainty }\end{array}$ \\
\hline $\mathbf{1 . 0 0 E + 0 4}$ & $\mathbf{1 . 0 0 E + 0 5}$ & 0.5 & $110 \%$ & $1098 \%$ \\
$\mathbf{1 . 0 0 E + 0 5}$ & $\mathbf{6 . 0 0 E + 0 5}$ & 11.1 & $5 \%$ & $45 \%$ \\
$\mathbf{6 . 0 0 E + 0 5}$ & $\mathbf{1 . 0 0 E + 0 6}$ & 18.6 & $3 \%$ & $24 \%$ \\
$\mathbf{1 . 0 0 E + 0 6}$ & $\mathbf{2 . 5 0 E + 0 6}$ & 24.8 & $2 \%$ & $14 \%$ \\
$\mathbf{2 . 5 0 E + 0 6}$ & $\mathbf{1 . 0 0 E + 0 7}$ & 39.0 & $1 \%$ & $6 \%$ \\
$\mathbf{1 . 0 0 E + 0 7}$ & $\mathbf{I n f}$ & 6.0 & $8 \%$ & $5 \%$ \\
\hline
\end{tabular}




\subsection{SECTION SUMMARY}

The responses of the various detectors are summarized. Notably, consistent differences in the fast portion of the neutron spectra were observed for the various shielding samples. Some features were observed on gamma ray spectra. The non-moderating shielding samples may have produced high energy secondary gammas at low intensity. Approximate foil responses were determined. The bubble spectrometer appears to be more accurate at higher energies. 


\section{SOURCE INTENSITY FOR GOOD MEASUREMENTS}

Ideally, source intensity would be calculated beforehand and scaled for various positions. If a source intensity estimate is not provided prior to experiment execution, then the source intensity should be increased until counters provide reasonable count rates, and the intensity should be held until reasonably good count statistics are obtained (probably no more than 10 minutes). Then, if a second detector still does not provide good count statistics, the intensity or count time can be reasonably increased until it does, making sure not to exceed detector ratings. The measurement location in the room return shield was chosen to be $3.6 \mathrm{~m}$ from the source for this evaluation. Alternate locations for the room return shield would be scaled up or down using a point source approximation.

Table 5 shows the maximum assembly power that electronic detectors can reasonably work with. It is expected that the EJ-309 and the BGO scintillators can handle between 20,000 and 100,000 counts per second. Count rates per joule of assembly fission energy were computed as flux multiplied by elastic scattering cross section, or gamma interaction probability, so they are only estimates. They are based on results from the simulations that are explicitly modeling the BGO and EJ-309 interactions in the detectors. It was assumed that each interaction above $10 \mathrm{keV}$ results in a count. $10 \mathrm{keV}$ is a low energy for these detectors, so this should be somewhat conservative.

Table 5. Detector performance range and corresponding source intensity

\begin{tabular}{|r|r|r|l} 
Detector & $\begin{array}{l}\text { Maximum detector } \\
\text { range (counts/s) }\end{array}$ & $\begin{array}{l}\text { conversion } \\
\text { (counts/J) }\end{array}$ & $\begin{array}{l}\text { Maximum assembly } \\
\text { power (W) }\end{array}$ \\
\hline EJ 309 & 100000 & $1 \mathrm{E}+06$ & \\
\hline BGO & 20000 & $5 \mathrm{E}+04$ & 0.1 \\
& & & 0.4
\end{tabular}

Table 5 shows that source intensities of 0.01 to 0.1 watts (4E-11 to $4 \mathrm{E}-10$ amps on the intermediate power detector) should produce high but attainable readings for the fast neutron detector and the gamma detector. This source intensity may be easier to obtain in subcritical mode using a driver neutron source. Notably, no driver source was modeled, but the multiplication factor (keff) will be close to 1 , so the source should produce only a small fraction of the neutrons. This prediction was made assuming that all recoils above $100 \mathrm{keV}$ would be counted, which is a best estimate. It also assumes that the shielding sample consists of $10 \mathrm{~cm}$ of $\mathrm{SiO} 2$, which blocks half the neutrons and half the gammas, so it is not worst case, but it provides a reasonable order of magnitude estimate. It is also assumed that all photon interactions are being detected, resulting in roughly 20000 counts per second for the BGO detector, again to provide an order of magnitude estimate.

Table 6 gives effective operating ranges for various integral detectors. Applicable units defining the effective detector range are different for different detectors. Detectors measuring dose, have units in dose, and activation detectors have units listed in $\mathrm{Bq}$ of activation. Responses per joule of fission in the source are determined from computations and the necessary number of fissions (expressed in joule of thermal energy) are shown. These are taken as a minimum of the detector range divided by the minimum conversion, and the maximum divided by the maximum, so the exposure range is somewhat narrower than it could be. The range for bubble spectrometers is based on forming between 2 and 100 bubbles. Glass in gamma detector, Alumina TLD, and diode limits were provided by a dosimetrist at IRSN [28]. Foils are assumed to require $50 \mathrm{~Bq}$ to be countable. This is based on counts in IER 147 [20] for the sulfur pellet, but it is by no means a precise number, and the value may be lower. Dose per $\mathrm{J}$ of operation for the bubble spectrometer is determined by modeling. The Glass, TLD, etc., ranges are estimated from the flux in simulated TLDs or scintillators and the 1977 dose conversion factor. 
Table 7 shows the necessary source durations and intensities for the lower and upper limits of the detector using the exposure column values of the previous table. Values that appear reasonable are highlighted green while values that appear possible but difficult are highlighted yellow. These are not precise predictions; they are simply a means to down select detectors and exposure levels to promote good measurements.

As an example of how Table 6 fission intensities are computed, the CaF2 TLD is considered. Multiplying the ANSI/ANS-6.1.1-1977 flux-to-dose conversion factor by the flux for each energy group in a simulation and summing over energy groups gives a total dose rate. The simulation was performed with a fission source with $1 \mathrm{~J}$ of fissions. The computed dose rate is approximately $0.07 \mathrm{mGy} /$ hour at a power level of 1 watt for a TLD behind a shielding sample. As shown in Table 7 at the 0.01 -watt operating level, this is approximately $0.7 \mu \mathrm{Gy} /$ hour, so higher power is needed to obtain a TLD response. Cross calibration may thus be intractable using a TLD due to count rate limits.

Table 6. Detector performance ranges and corresponding source intensity

\begin{tabular}{|c|c|c|c|c|c|c|c|}
\hline \multirow[b]{2}{*}{ Detector } & \multirow{2}{*}{$\begin{array}{c}\text { Range } \\
\text { units }\end{array}$} & \multicolumn{2}{|c|}{ Range } & \multicolumn{2}{|c|}{ Range units per $\mathrm{J}$ of fission } & \multicolumn{2}{|c|}{ Fission intensity $(\mathrm{J})$} \\
\hline & & Minimum & Maximum & Minimum & Maximum & Minimum & Maximum \\
\hline Bubble Spect $10 \mathrm{keV}$ & $\mu \mathrm{Sv}$ & 13 & 673 & $1.89 \mathrm{E}-01$ & $1.00 \mathrm{E}+00$ & 71 & 673 \\
\hline Bubble Spect $100 \mathrm{keV}$ & $\mu \mathrm{Sv}$ & 13 & 673 & $1.88 \mathrm{E}-01$ & $9.96 \mathrm{E}-01$ & 71 & 676 \\
\hline Bubble Spect $600 \mathrm{keV}$ & $\mu \mathrm{Sv}$ & 13 & 673 & $1.71 \mathrm{E}-01$ & $8.65 \mathrm{E}-01$ & 78 & 778 \\
\hline Bubble Spect $1 \mathrm{MeV}$ & $\mu \mathrm{Sv}$ & 13 & 673 & $1.41 \mathrm{E}-01$ & $6.43 \mathrm{E}-01$ & 94 & 1048 \\
\hline Bubble Spect $2.5 \mathrm{MeV}$ & $\mu S v$ & 13 & 673 & $9.32 \mathrm{E}-02$ & $3.82 \mathrm{E}-01$ & 143 & 1762 \\
\hline Bubble Spect $10 \mathrm{MeV}$ & $\mu \mathrm{Sv}$ & 13 & 673 & $9.36 \mathrm{E}-03$ & $3.94 \mathrm{E}-02$ & 1425 & 17086 \\
\hline RPL Glass in detector chamber & $\mu G y$ & 50 & $1.00 \mathrm{E}+08$ & 5.00E-03 & $1.60 \mathrm{E}-02$ & 10000 & 6250000000 \\
\hline Alumina TLD in detector chamber & $\mu G y$ & $1.00 \mathrm{E}+05$ & & $5.00 \mathrm{E}-03$ & $1.60 \mathrm{E}-02$ & 20000000 & \\
\hline CaF2 TLD & $\mu G y$ & $3.60 \mathrm{E}+02$ & & $5.00 \mathrm{E}-03$ & & 72000 & \\
\hline Diode in detector chamber & $\mu G y$ & $2.00 \mathrm{E}+04$ & $5.00 \mathrm{E}+06$ & $2.36 \mathrm{E}-01$ & $2.36 \mathrm{E}-01$ & 84746 & 21186441 \\
\hline RPL Glass on front of RRS & $\mu G y$ & 50 & $1.00 \mathrm{E}+08$ & 7.30E-02 & $9.90 \mathrm{E}-02$ & 685 & 1010101010 \\
\hline Alumina TLD on front of RRS & $\mu G y$ & 100000 & & 7.30E-02 & $9.90 \mathrm{E}-02$ & 1369863 & \\
\hline CaF2 TLD & $\mu G y$ & $3.60 \mathrm{E}+02$ & & 7.30E-02 & & 4932 & \\
\hline gold & $\mathrm{Bq}$ & 50 & & $1.14 \mathrm{E}-04$ & $1.53 \mathrm{E}-03$ & 439968 & \\
\hline sulfur & $\mathrm{Bq}$ & 50 & & $1.60 \mathrm{E}-06$ & 7.90E-06 & 31197461 & \\
\hline indium $116 \mathrm{~m}$ & $\mathrm{~Bq}$ & 50 & & $2.01 \mathrm{E}-03$ & $3.20 \mathrm{E}-02$ & 24849 & \\
\hline gold (cd shielded) & $\mathrm{Bq}$ & 50 & & $1.02 \mathrm{E}-04$ & 4.25E-04 & 489031 & \\
\hline indium (b10 shielded) & $\mathrm{Bq}$ & 50 & & 8.51E-04 & 4.65E-03 & 58735 & \\
\hline copper (b10 shielded) & $\mathrm{Bq}$ & 50 & & $5.45 \mathrm{E}-05$ & 2.37E-04 & 916947 & \\
\hline Indium $115 \mathrm{~m}$ & $\mathrm{~Bq}$ & 50 & & $2.98 \mathrm{E}-04$ & $1.36 \mathrm{E}-03$ & 167606 & \\
\hline
\end{tabular}


Table 7. Times to obtain a workable reading at various power levels (or number of bursts corresponding to edges of detector ranges)

\begin{tabular}{|c|c|c|c|c|c|c|}
\hline \multirow{2}{*}{ Detector } & \multicolumn{2}{|c|}{ minutes at $0.1 \mathrm{~W}$} & \multicolumn{2}{|c|}{ Minutes at $10 \mathrm{~W}$} & \multicolumn{2}{|c|}{ Number of $70^{\circ} \mathrm{C}$ bursts } \\
\hline & Minimum & Maximum & Minimum & Maximum & Minimum & Maximum \\
\hline Bubble Spectrometer $10 \mathrm{keV}$ & 12 & 112 & 0.1 & 1.1 & 0.00 & 0.00 \\
\hline Bubble Spectrometer $100 \mathrm{keV}$ & 12 & 113 & 0.1 & 1.1 & 0.00 & 0.00 \\
\hline Bubble Spectrometer $600 \mathrm{keV}$ & 13 & 130 & 0.1 & 1.3 & 0.00 & 0.00 \\
\hline Bubble Spectrometer $1 \mathrm{MeV}$ & 16 & 175 & 0.2 & 1.7 & 0.00 & 0.00 \\
\hline Bubble Spectrometer $2.5 \mathrm{MeV}$ & 24 & 294 & 0.2 & 2.9 & 0.00 & 0.00 \\
\hline Bubble Spectrometer $10 \mathrm{MeV}$ & 238 & 2848 & 2.4 & 28.5 & 0.00 & 0.03 \\
\hline RPL Glass in detector chamber & 1667 & 1041666667 & 16.7 & 10416666.7 & 0.02 & 11363.64 \\
\hline Alumina TLD in detector chamber & 3333333 & 0 & 33333.3 & & 36.36 & \\
\hline $\mathrm{CaF}_{2}$ TLD & 12000 & & 120.0 & & 0.13 & \\
\hline Diode in detector chamber & 14124 & 3531073 & 141.2 & 35310.7 & 0.15 & 38.52 \\
\hline RPL Glass on front of RRS & 114 & 168350168 & 1.1 & 1683501.7 & 0.00 & 1836.55 \\
\hline Alumina TLD on front of RRS & 228311 & & 2283.1 & & 2.49 & \\
\hline $\mathrm{CaF}_{2}$ TLD & 822 & & 8.2 & & 0.01 & \\
\hline Gold & 73328 & & 733.3 & & 0.80 & \\
\hline Sulfur & 5199577 & & 51995.8 & & 56.72 & \\
\hline Indium $116 \mathrm{~m}$ & 4141 & & 41.4 & & 0.05 & \\
\hline gold (Cd shielded) & 81505 & & 815.1 & & 0.89 & \\
\hline indium (B10 shielded) & 9789 & & 97.9 & & 0.11 & \\
\hline copper (B10 shielded) & 152824 & & 1528.2 & & 1.67 & \\
\hline Indium $115 \mathrm{~m}$ & 27934 & & 279.3 & & 0.30 & \\
\hline
\end{tabular}




\section{SENSTIVITY TO PERTURBATIONS IN GEOMETRY AND DENSITIES AND TOTAL UNCERTAINTY}

A perturbation study was performed on a baseline model to gauge biases and uncertainties from the materials, densities, and geometries that are likely to affect measured values. The principal objective was to understand the impact of dimensional tolerances and other variations to determine if any are especially important for the quality of the benchmark. A formal perturbation analysis will be performed in CED-4, so the goal is to identify uncertainties that need to be managed and to obtain an approximate total uncertainty. The uncertainty study was subdivided as follows: (1) study of uncertainties arising from material and geometry unknowns far from the source (Section 5.1), (2) material and geometry assumptions near the source (Section 5.2), (3) source intensity uncertainty (Section 5.3), and (4) detector uncertainties. The uncertainties on measurements resulting from detector unknowns were assumed based on typical detector performance, whereas uncertainties on measurements resulting from geometry and material unknowns were evaluated using MAVRIC. The near and away-from source perturbations were performed separately, primarily because of workflow, and not for any technical reason. The perturbations on material and geometry away from the source are presented first, followed by perturbations on material and geometry near the source. Finally, the total effect of the perturbations, the uncertainty of source intensity, and detector uncertainty are combined to show the expected uncertainty of the benchmark experiment.

\subsection{PERTURBATION SET I: UNCERTAINTIES FAR FROM THE SOURCE}

\subsubsection{Introduction to Evaluation of Uncertainties Far from the Source}

The far-from-source sensitivity evaluations were conducted for a $\mathrm{SiO}_{2}$ shielding sample. Table 8 shows perturbations and scaling factors. Because some geometric uncertainties are small, they cannot be expected to produce noticeable changes for a given level of Monte Carlo uncertainty. Therefore, a scaled up geometric perturbation that was substantially larger than the Monte Carlo uncertainty was modeled, and the resulting changes were scaled back down by the same factor.

Most uncertainties in geometry listed in Table 8 are based on the intuition of the authors, because the ultimate uncertainties will be established when equipment is fabricated, and the experiment is performed. For example, fabricators may by default manufacture to better tolerances, or they may have difficulty meeting some other tolerance. Similarly, experimenters may be able to better determine position than is credited here, or there may be some unanticipated additional form of uncertainty.

The expected uncertainties in aim and room return positioning require some justification. Distance from the source to the room return (RR) shield and aiming of the RR longitudinal axis in a direction normal to the outer surface of the source were two impactful parameters, so tighter tolerances are specified for these. For example, azimuthal and zenith angles are specified at 0.3 degrees. This is the angle subtended by a $5 \mathrm{~cm}$ wide target at $10 \mathrm{~m}$. Additional planning may be needed, but an angular tolerance of 0.3 degrees should be achievable with adequate preparation. A human can align their eye and the tip of their thumb to test this assertion. From intuition, a $2 \mathrm{~cm}$ distance reproducibility is accomplished with a sharp line on the floor to align the room return shield assembly. Several factors were also found to affect neutron measurement intensity such as detector placement. These were not used in specifying perturbation uncertainties.

Due to benchmark quantities being comparisons of treatments to baseline values, many parameter biases are expected to cancel with the biases in the baseline case. For example, if a detector always reads $10 \%$ high, because the benchmark quantities are defined as treatment divided by baseline, that $10 \%$ bias 
cancels out to a degree. No detailed analysis of biases aside from determination of leakage past the shielding sample was performed.

Note that cases 017 thru 025 altered detector positions in the room return shield according to cylindrical coordinates, with the axis of the cylinder being used as the basis for rotation. They did not alter the aim of the detectors, because they have little ability to precisely be aimed.

Table 8. Overview of geometry and density perturbations performed with scaling multipliers

\begin{tabular}{|c|c|c|c|c|c|}
\hline $\begin{array}{c}\text { Case } \\
\text { number }\end{array}$ & Case & $\begin{array}{l}\text { Perturbation } \\
\text { for case }\end{array}$ & $\begin{array}{l}\text { Expected } \\
\text { parameter } \\
\text { uncertainty }\end{array}$ & $\begin{array}{c}\text { Unit for } \\
\text { perturbation } \\
\text { and } \\
\text { uncertainty } \\
\end{array}$ & $\begin{array}{l}\text { Scaling } \\
\text { multiplier } \\
\text { (unitless) }\end{array}$ \\
\hline 000 & $\begin{array}{l}\text { Average of perturbations (in lieu of } \\
\text { baseline) }\end{array}$ & - & - & - & - \\
\hline 001 & Case with cavity increased in size by $1 \mathrm{~cm}$ & 1 & 0.2 & $\mathrm{~cm}$ & 0.2 \\
\hline 002 & Case with streaming channels closed off & -100 & -100 & $\%$ & 1 \\
\hline 003 & $\begin{array}{l}\text { Case with polyethylene density in room } \\
\text { return shield reduced } 10 \%\end{array}$ & -10 & -2 & $\%$ & 0.2 \\
\hline 004 & $\begin{array}{l}\text { Case with lead density in room return } \\
\text { shield reduced } 10 \%\end{array}$ & -10 & -2 & $\%$ & 0.2 \\
\hline 005 & $\begin{array}{l}\text { Case with polyethylene density in flux trap } \\
\text { reduced } 10 \%\end{array}$ & -10 & -1 & $\%$ & 0.1 \\
\hline 006 & $\begin{array}{l}\text { Case with lead density in flux trap reduced } \\
10 \%\end{array}$ & -10 & -1 & $\%$ & 0.1 \\
\hline 007 & $\begin{array}{l}\text { Case with room return shield offset by } 10 \\
\mathrm{~cm} \text { in } \mathrm{x} \text { direction (no change in aim) }\end{array}$ & 10 & 2 & $\mathrm{~cm}$ & 0.2 \\
\hline 010 & $\begin{array}{l}\text { Case with room return shield azimuth aim } \\
\text { offset by } 1 \text { degree }\end{array}$ & 1 & 0.3 & deg & 0.3 \\
\hline 011 & $\begin{array}{l}\text { Case with room return shield aim zenith } \\
\text { offset by } 1 \text { degree }\end{array}$ & 1 & 0.3 & deg & 0.3 \\
\hline 017 & $\begin{array}{l}\text { Case with gamma detector position } \\
\text { adjusted } 5 \text { degrees in azimuth from RR } \\
\text { cylinder axis }\end{array}$ & 5 & 5 & deg & 1 \\
\hline 018 & $\begin{array}{l}\text { Case with gamma detector position radius } \\
\text { decreased } 3 \mathrm{~mm} \text { from RR cylinder axis }\end{array}$ & -0.3 & -0.2 & $\mathrm{~cm}$ & 0.666667 \\
\hline 019 & $\begin{array}{l}\text { Case with gamma detector position moved } \\
0.2 \mathrm{~cm} \text { from the sample }\end{array}$ & 0.5 & 0.2 & $\mathrm{~cm}$ & 0.4 \\
\hline 020 & $\begin{array}{l}\text { Case with fast neutron detector position } \\
\text { adjusted } 5 \text { degrees in azimuth from RR } \\
\text { cylinder axis }\end{array}$ & 5 & 5 & deg & 1 \\
\hline 021 & $\begin{array}{l}\text { Case with fast neutron detector position } \\
\text { radius decreased } 3 \mathrm{~mm} \text { from RR cylinder } \\
\text { axis }\end{array}$ & -0.3 & -0.2 & $\mathrm{~cm}$ & 0.666667 \\
\hline 022 & $\begin{array}{l}\text { Case with fast neutron detector position } \\
\text { moved } 0.2 \mathrm{~cm} \text { from the sample }\end{array}$ & 0.5 & 0.2 & $\mathrm{~cm}$ & 0.4 \\
\hline 023 & $\begin{array}{l}\text { Case with thermal neutron detector position } \\
\text { adjusted } 5 \text { degrees in azimuth from RR } \\
\text { cylinder axis }\end{array}$ & 5 & 5 & deg & 1 \\
\hline 024 & $\begin{array}{l}\text { Case with thermal neutron detector position } \\
\text { radius decreased } 3 \mathrm{~mm} \text { from RR cylinder } \\
\text { axis }\end{array}$ & -0.3 & -0.2 & $\mathrm{~cm}$ & 0.666667 \\
\hline 025 & $\begin{array}{l}\text { Case with thermal neutron detector moved } \\
0.2 \mathrm{~cm} \text { from the sample }\end{array}$ & 0.5 & 0.2 & $\mathrm{~cm}$ & 0.4 \\
\hline 026 & Case with detectors having $1 \mathrm{~cm}$ liner & -100 & -3 & $\%$ & 0.03 \\
\hline 027 & Case with no room & & & & 1 \\
\hline
\end{tabular}

The models used for neutrons included a flux trap which is not included in later iterations (gammas and near-source perturbations) because it was determined that inclusion of a flux trap did not make much of a difference in the calculated parameter uncertainty. The case numbers 005 and 006 were for cases associated with a flux trap which included polyethylene rings near the shielding sample. The flux trap was 
originally included to prevent streaming around some samples. It does not lie along the primary path of radiation, nor did it line the detector chamber, so uncertainties are still expected to be similar those shown here. Note that all uncertainties discussed are 1-sigma. In the next evaluation of these sensitivities, the updated model will be used.

\subsubsection{Neutron Results to perturbations far from the source}

Sensitivity of neutron measurement to perturbations on materials and geometry far from the source was observed for several perturbations listed in Table 8, including cavity radius, lead density, room return shield placement, room return angles, and neutron detector axial placement. Of these factors, cavity radius and room return shield placement appear to introduce the most uncertainty. The first case (000) represents the baseline nominal case against which the perturbations are compared. Some detector location perturbations also produce large responses. This may indicate that the detectors need to be reliably mounted in a known location, and not simply laid in the room return shield or clipped to something. This may also be an artifact of the way variance reduction methods were used in the simulation.

Table 9 shows the results for each perturbation for neutron flux through the fast detector for energies greater than $100 \mathrm{keV}$. Some cells are color-coded to reflect magnitude of the number in each cell of the table, with bluer being a lower number and redder being a higher number. The perturbations resulted in a total geometric uncertainty of roughly $3.3 \%$ for a given measurement - not including source and detector uncertainty. The total geometric uncertainty was computed as the root sum of squares of all scaled relative differences introduced by the perturbations considered. A longer description of the computation follows.

To calculate total geometric uncertainty, the first step is to compute the relative response $\left(R_{i}\right)$ to a perturbation $\left(P_{i}\right)$ as

$$
R_{i}=\frac{P_{i}}{B}-1
$$

where B represents baseline. The absolute uncertainty $\left(\sigma_{R_{i}}\right)$ of $R_{i}$ from Monte Carlo (MC) uncertainty is computed as

$$
\sigma_{R_{i}}=\frac{P_{i}}{B} \sqrt{\left(\frac{\sigma_{P_{i}}}{P_{i}}\right)^{2}+\left(\frac{\sigma_{B}}{B}\right)^{2}}
$$

where $\sigma$ is the standard deviation of the quantity in the subscript. Note that $\sigma_{R_{i}}$ is not relative uncertainty of the relative difference, but rather absolute uncertainty of relative difference.

The relative difference from a perturbation in Eq. (3) is then multiplied by the scaling factor $\left(S_{i}\right)$ to get the relative difference from parameter uncertainty $\left(\mathrm{r}_{\mathrm{i}}\right)$. The perturbations, parameter uncertainties, and scaling multipliers are listed in Table 8. The same calculation is performed for the measure of Monte Carlo uncertainty $\left(\sigma_{r_{i}}\right)$.

The final total geometric uncertainty from all the perturbations is then computed as the square root of the sum of square of all relative differences from parameter uncertainties $\left(r_{i}\right)$ and is $3.3 \%$. 


\begin{tabular}{|c|c|c|c|c|c|c|c|c|}
\hline Number & Case & 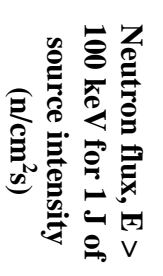 & 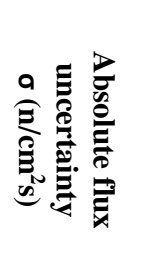 & 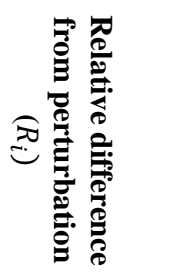 & 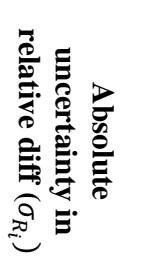 & 氡 & 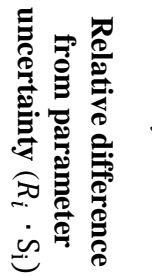 & 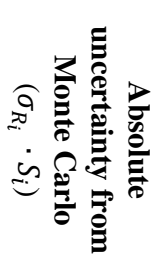 \\
\hline 000 & Base Case & 5286 & 25 & - & - & - & - & - \\
\hline 001 & $\begin{array}{l}\text { Case with cavity increased in size by } 1 \\
\mathrm{~cm}\end{array}$ & 5713 & 24 & $8.09 \%$ & $0.68 \%$ & 0.2 & $1.62 \%$ & $0.14 \%$ \\
\hline 002 & $\begin{array}{l}\text { Case with streaming channels closed } \\
\text { off }\end{array}$ & 5262 & 25 & $-0.45 \%$ & $0.66 \%$ & 1 & $-0.45 \%$ & $0.66 \%$ \\
\hline 003 & $\begin{array}{l}\text { Case with polyethylene density in } \\
\text { room return shield reduced } 10 \%\end{array}$ & 5303 & 26 & $0.31 \%$ & $0.68 \%$ & 0.2 & $0.06 \%$ & $0.14 \%$ \\
\hline 004 & $\begin{array}{l}\text { Case with lead density in room return } \\
\text { shield reduced } 10 \%\end{array}$ & 5277 & 27 & $-0.18 \%$ & $0.69 \%$ & 0.2 & $-0.04 \%$ & $0.14 \%$ \\
\hline 005 & $\begin{array}{l}\text { Case with polyethylene density in flux } \\
\text { trap reduced } 10 \%\end{array}$ & 5231 & 24 & $-1.05 \%$ & $0.65 \%$ & 0.1 & $-0.10 \%$ & $0.07 \%$ \\
\hline 006 & $\begin{array}{l}\text { Case with lead density in flux trap } \\
\text { reduced } 10 \%\end{array}$ & 5307 & 26 & $0.40 \%$ & $0.68 \%$ & 0.1 & $0.04 \%$ & $0.07 \%$ \\
\hline 007 & $\begin{array}{l}\text { Case with room return shield offset by } \\
10 \mathrm{~cm} \text { in } x \text { direction (no change in } \\
\text { aim) }\end{array}$ & 4899 & 23 & $-7.33 \%$ & $0.62 \%$ & 0.2 & $-1.47 \%$ & $0.12 \%$ \\
\hline 010 & $\begin{array}{l}\text { Case with room return shield azimuth } \\
\text { aim offset by } 1 \text { degree }\end{array}$ & 5295 & 27 & $0.17 \%$ & $0.69 \%$ & 0.3 & $0.05 \%$ & $0.21 \%$ \\
\hline 011 & $\begin{array}{l}\text { Case with room return shield zenith } \\
\text { offset by } 1 \text { degree }\end{array}$ & 5179 & 23 & $-2.03 \%$ & $0.63 \%$ & 0.3 & $-0.61 \%$ & $0.19 \%$ \\
\hline 017 & $\begin{array}{l}\text { Case with gamma detector adjusted } 5 \\
\text { degrees in azimuth from RR axis }\end{array}$ & 5316 & 27 & $0.56 \%$ & $0.69 \%$ & 1 & $0.56 \%$ & $0.69 \%$ \\
\hline 018 & $\begin{array}{l}\text { Case with gamma detector radius } \\
\text { decreased } 3 \mathrm{~mm} \text { from RR axis }\end{array}$ & 5246 & 24 & $-0.77 \%$ & $0.66 \%$ & 0.667 & $-0.51 \%$ & $0.44 \%$ \\
\hline 019 & $\begin{array}{l}\text { Case with gamma detector moved } 0.2 \\
\mathrm{~cm} \text { from the sample }\end{array}$ & 5292 & 30 & $0.12 \%$ & $0.73 \%$ & 0.4 & $0.05 \%$ & $0.29 \%$ \\
\hline 020 & $\begin{array}{l}\text { Case with fast neutron detector } \\
\text { adjusted } 5 \text { degrees in azimuth from RR } \\
\text { axis }\end{array}$ & 5243 & 24 & $-0.81 \%$ & $0.65 \%$ & 1 & $-0.81 \%$ & $0.65 \%$ \\
\hline 021 & $\begin{array}{l}\text { Case with fast neutron detector radius } \\
\text { decreased } 3 \mathrm{~mm} \text { from RR axis }\end{array}$ & 5221 & 24 & $-1.23 \%$ & $0.65 \%$ & 0.667 & $-0.82 \%$ & $0.43 \%$ \\
\hline 022 & $\begin{array}{l}\text { Case with fast neutron detector moved } \\
0.2 \mathrm{~cm} \text { from the sample }\end{array}$ & 5095 & 23 & $-3.61 \%$ & $0.64 \%$ & 0.4 & $-1.44 \%$ & $0.25 \%$ \\
\hline 023 & $\begin{array}{l}\text { Case with thermal neutron detector } \\
\text { adjusted } 5 \text { degrees in azimuth from RR } \\
\text { axis }\end{array}$ & 5240 & 24 & $-0.86 \%$ & $0.66 \%$ & 1 & $-0.86 \%$ & $0.66 \%$ \\
\hline 024 & $\begin{array}{l}\text { Case with thermal neutron detector } \\
\text { radius decreased } 3 \mathrm{~mm} \text { from RR axis }\end{array}$ & 5231 & 23 & $-1.05 \%$ & $0.64 \%$ & 0.667 & $-0.70 \%$ & $0.43 \%$ \\
\hline 025 & $\begin{array}{l}\text { Case with thermal neutron detector } \\
\text { moved } 0.2 \mathrm{~cm} \text { from the sample }\end{array}$ & 5301 & 23 & $0.28 \%$ & $0.64 \%$ & 0.4 & $0.11 \%$ & $0.26 \%$ \\
\hline 026 & Case with detectors having $1 \mathrm{~cm}$ liner & 5232 & 23 & $-1.01 \%$ & $0.64 \%$ & 0.03 & $-0.03 \%$ & $0.02 \%$ \\
\hline 027 & Case with no room & 5254 & 22 & $-0.61 \%$ & $0.63 \%$ & 1 & $-0.61 \%$ & $0.63 \%$ \\
\hline \multicolumn{9}{|c|}{ Propagated uncertainty } \\
\hline
\end{tabular}

\subsubsection{Gamma Results to perturbations far from the source}

The gamma result had a total far-from-source geometric uncertainty of $8.3 \%$, which was computed as the square root of the sum of the squares of all the relative differences from all the perturbations shown in Table 10 in a calculation like that performed for neutrons. This was performed using a set of calculations separate from those used for the neutron calculations. No flux trap was assumed, so cases 5 and 6 were 
subsumed by cases 3 and 4 . The cases were set to accelerate convergence for gamma tallies, specifically. Grey rows used reduced geometries which did not include the entire room to save on computational resources. The justification for this is that case 27 shows that little effect is introduced from the room. The baseline with reduced geometry (grey) was used as a basis of comparison for the other cases with reduced geometries (other grey rows). The baseline with full geometry (black) was used as a basis of comparison for the other cases with full geometries.

Table 10. Gamma sensitivities to perturbations

\begin{tabular}{|c|c|c|c|c|c|c|c|c|}
\hline Number & Case & 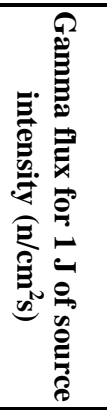 & 疍 & 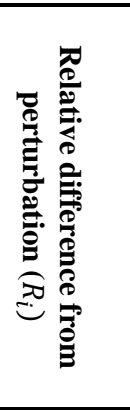 & 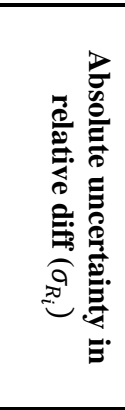 & 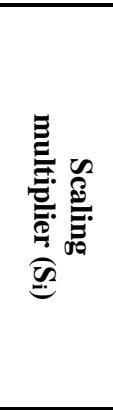 & 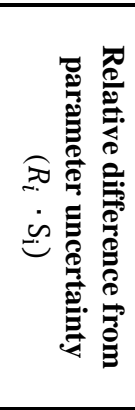 & 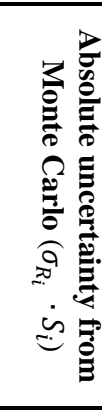 \\
\hline 000 & Baseline (average of 3 runs) & 1714 & 14 & & & & & \\
\hline 000 & $\begin{array}{l}\text { Baseline reduced (average of } 4 \text { runs) } \\
\text { Case with cavity increased in size by } 1\end{array}$ & 1719 & 15 & & & & & \\
\hline 001 & $\mathrm{~cm}$ Case with streaming channels closed & 1845 & 46 & $7.34 \%$ & $2.81 \%$ & 0.2 & $1.47 \%$ & $0.56 \%$ \\
\hline 002 & $\begin{array}{l}\text { Case with streaming channels closed } \\
\text { off }\end{array}$ & 1790 & 80 & $4.45 \%$ & $4.76 \%$ & 1 & $4.45 \%$ & $4.76 \%$ \\
\hline 003 & $\begin{array}{l}\text { Case with polyethylene density in } \\
\text { room return shield reduced } 10 \% \\
\text { Case with lead density in room return }\end{array}$ & 1863 & 101 & $8.70 \%$ & $5.93 \%$ & 0.2 & $1.74 \%$ & $1.19 \%$ \\
\hline 004 & $\begin{array}{l}\text { shield reduced } 10 \% \\
\text { Case with room return shield offset by }\end{array}$ & 1640 & 26 & $-4.31 \%$ & $1.68 \%$ & 0.2 & $-0.86 \%$ & $0.34 \%$ \\
\hline 007 & $10 \mathrm{~cm}$ in $\mathrm{x}$ direction (no change in aim) & 1565 & 21 & $-8.97 \%$ & $1.44 \%$ & 0.2 & $-1.79 \%$ & $0.29 \%$ \\
\hline 010 & $\begin{array}{l}\text { Case with room return shield azimuth } \\
\text { aim offset by } 1 \text { degree } \\
\text { Case with room return shield zenith }\end{array}$ & 1703 & 23 & $-0.95 \%$ & $1.57 \%$ & 0.3 & $-0.29 \%$ & $0.47 \%$ \\
\hline 011 & $\begin{array}{l}\text { offset by } 1 \text { degree } \\
\text { Case with gamma detector adjusted } 5\end{array}$ & 1731 & 72 & $0.68 \%$ & $4.26 \%$ & 0.3 & $0.20 \%$ & $1.28 \%$ \\
\hline 017 & $\begin{array}{l}\text { degrees in azimuth from } \mathrm{RR} \text { axis } \\
\text { Case with gamma detector radius }\end{array}$ & 1673 & 23 & $-2.71 \%$ & $1.55 \%$ & 1 & & $1.55 \%$ \\
\hline 018 & $\begin{array}{l}\text { decreased } 3 \mathrm{~mm} \text { from } \mathrm{RR} \text { axis } \\
\text { Case with gamma detector moved } 0.2\end{array}$ & 1705 & 19 & $-0.83 \%$ & $1.39 \%$ & 0.667 & $-0.55 \%$ & $0.93 \%$ \\
\hline 019 & $\begin{array}{l}\text { cm from the sample } \\
\text { Case with fast neutron detector } \\
\text { adjusted } 5 \text { degrees in azimuth from RR }\end{array}$ & 1848 & 125 & $7.52 \%$ & $7.33 \%$ & 0.4 & & $2.93 \%$ \\
\hline 020 & $\begin{array}{l}\text { axis } \\
\text { Case with fast neutron detector radius }\end{array}$ & 1706 & 26 & $-0.76 \%$ & $1.75 \%$ & 1 & $-0.76 \%$ & $1.75 \%$ \\
\hline 021 & $\begin{array}{l}\text { decreased } 3 \mathrm{~mm} \text { from } \mathrm{RR} \text { axis } \\
\text { Case with fast neutron detector moved }\end{array}$ & 1784 & 59 & $3.77 \%$ & $3.54 \%$ & 0.667 & $2.51 \%$ & $2.36 \%$ \\
\hline 022 & $\begin{array}{l}0.2 \mathrm{~cm} \text { from the sample } \\
\text { Case with thermal neutron detector } \\
\text { adjusted } 5 \text { degrees in azimuth from RR }\end{array}$ & 1676 & 18 & $-2.54 \%$ & $1.35 \%$ & 0.4 & $-1.01 \%$ & $0.54 \%$ \\
\hline 023 & axis & 1655 & 19 & $-3.72 \%$ & $1.36 \%$ & 1 & $.72 \%$ & $1.36 \%$ \\
\hline 024 & $\begin{array}{l}\text { Case with thermal neutron detector } \\
\text { radius decreased } 3 \mathrm{~mm} \text { from RR axis } \\
\text { Case with thermal neutron detector }\end{array}$ & 1738 & 29 & $1.08 \%$ & $1.90 \%$ & 0.667 & $0.72 \%$ & $1.27 \%$ \\
\hline 025 & moved $0.2 \mathrm{~cm}$ from the sample & 1688 & 27 & $-1.79 \%$ & $1.76 \%$ & 0.4 & $-0.72 \%$ & $0.71 \%$ \\
\hline 026 & Case with detectors having $1 \mathrm{~cm}$ liner & 1744 & 21 & $1.43 \%$ & $1.48 \%$ & 0.03 & $0.04 \%$ & $0.04 \%$ \\
\hline 027 & Case with no room & 1730 & 31 & $0.97 \%$ & $1.99 \%$ & 1 & $0.97 \%$ & $1.99 \%$ \\
\hline
\end{tabular}


The case with gamma channels closed off for streaming, and the case with detector radius changes may simply be Monty Carlo uncertainty. A previous iteration did not reveal streaming channels as a source of bias or uncertainty. Furthermore, for a particle to stream through the long, narrow channel, it would require a near-perfect incoming angle. It appears that thermal neutron detector placement may be a substantial source of uncertainty for gammas, and again a robust detector mounting arrangement should be used.

\subsection{PERTURBATION SET II: UNCERTAINTIES NEAR THE SOURCE}

The source is fission neutrons and photons from fast fission of ${ }^{235} \mathrm{U}$. The energies of the gammas can be modified by the depth of fission and other perturbations to the geometry around the source. Thus, examination of material and geometry source perturbations also inherently includes analysis of perturbations to the source spectrum. Specifically, if the distance fissions are from the assembly surface changes or are not precisely known, then the gamma energy spectrum emanated from the source will be changed. This is less of a concern for neutrons because (1) neutrons produced through fission are similar in energy to those causing fission, (2) downscatter is not pronounced for heavy isotopes such as the fuel, and (3) most absorptions in the fast spectrum cause fissions. Other geometry items were perturbed around the source to attempt to gauge effects of geometric uncertainties on the measurement.

Source perturbations were performed in a different manner than the perturbations on the room return shield. For the source, sensitivity of the dose reduction from a $\mathrm{SiO}_{2}$ shielding sample was evaluated with larger perturbations. However, for uncertainties far from the source, only the sensitivity of the dose itself to the geometry and density perturbations was evaluated. In both cases, the perturbations were exaggerated to capture the sensitivity with Monte Carlo, and then linearity was assumed to scale them to the expected values. Table 11 below shows the various cases used and the sizes of the perturbations. Most perturbations in the table are expressed in percent of some nominal value.

For the models in this section (Figure 27), an MCNP model of the Godiva IV assembly (left) was used to produce source spectra and to scale intensities. The model includes some corrections to the safety block shim, the safety block position, and the safety block volume loss. The model extends to the Tophat cover and includes the bottom box and base plate, but a surface tally on the surface of the fuel cylinder was taken to supply flux intensity and spectrum to the MAVRIC model (right), which uses an evenly distributed, isotropic source in the same volume as the surface tally. Elsewhere in this report, the MAVRIC model assumed a uniform source in a cylinder with the fuel's composition. This could be a gross approximation for benchmark modeling, but it was done to allow more rapid evaluation of sensitivities, and because the two-measurement benchmark quantity should be relatively insensitive to such an approximation. Benchmark models in CED-4 should use a mesh source map, and more precise clamps and base plate models should be examined than those used here. 

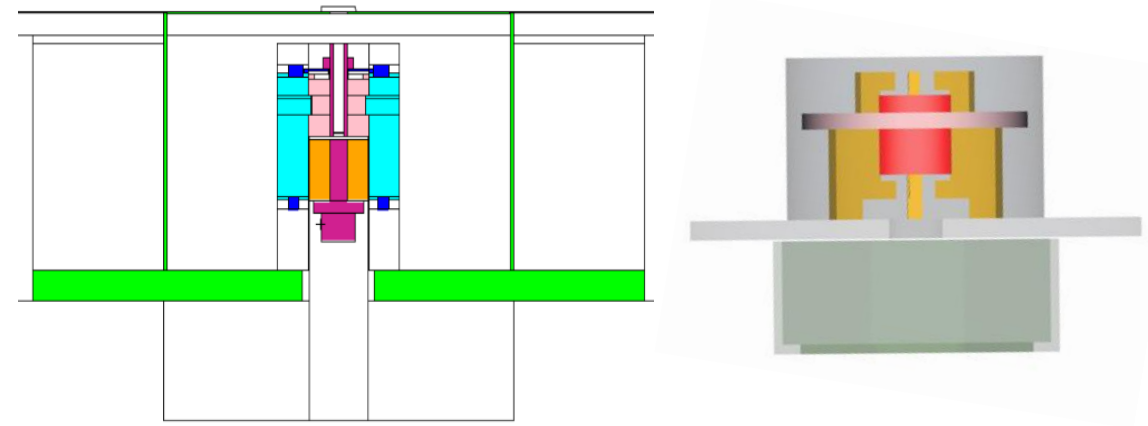

Figure 27. MCNP Godiva model (left), MAVRIC model (right).

Table 11. Perturbations of geometry and density near the source

\begin{tabular}{|c|c|c|c|c|c|}
\hline Number & Case & Description & Perturbation & $\begin{array}{l}\text { Expected } \\
\text { uncertainty } \\
\text { (based on } \\
\text { judgement) }\end{array}$ & $\begin{array}{c}\text { Scaling } \\
\text { factor }\left(S_{i}\right)\end{array}$ \\
\hline N101 & baseline & $\begin{array}{l}\text { More detailed source model, including band and } \\
\text { clamps, using an MCNP energy distribution for } \\
\text { neutrons and gammas uniformly distributed } \\
\text { through the source }\end{array}$ & & & \\
\hline N102 & bottom_box_density & $\begin{array}{l}\text { Bottom box (Lexan) density was perturbed in } \\
\text { MAVRIC and reduced by } 10 \% \text {. Baseline from } \\
\text { page } 47 \text { of Reference } 9 .\end{array}$ & $10 \%$ & $5 \%$ & $50.0 \%$ \\
\hline N103 & bottom_box_width & $\begin{array}{l}\text { Added } 8 \mathrm{~cm} \text { to each side (nominal is } 20-40 \mathrm{~cm} \text { ). } \\
\text { Baseline dimensions from Figure } 32 \text { of } \\
\text { Reference } 9 \text { "Plastic portion of contamination } \\
\text { shield, neglecting bolt holes and similar sized } \\
\text { detail. }\end{array}$ & $8.00 \mathrm{~cm}$ & 0.50 & $6.3 \%$ \\
\hline N104 & bottom_plate & $\begin{array}{l}\text { Bottom plate diameter was increased from } 44.45 \\
\text { to } 49.45 \mathrm{~cm} \text {, obscuring field of view of the } \\
\text { detector. Geometry from Figure } 63 \text { of Reference } \\
9 \text {, recognizing that the real geometry is triangular } \\
\text { and may need accounted for in CED- } 4 \text {. }\end{array}$ & $5 \mathrm{~cm}$ & 0.5 & $10.0 \%$ \\
\hline N105 & lorho. & $\begin{array}{l}\text { Reduced density of fuel in MCNP and MAVRIC } \\
\text { to } 90 \% \text { of nominal. MCNP model based on } \\
\text { Reference } 9 \text { using corrected shim plate. }\end{array}$ & $10 \%$ & $1 \%$ & $10.0 \%$ \\
\hline N106 & NoBandNoClamp & $\begin{array}{l}\text { Removed bands and clamps. Baseline uses } \\
\text { simplified geometry form Reference } 9 \text { page } 102 \\
\text { and band from Figure B-28, neglecting the bolt. }\end{array}$ & $100 \%$ & $20 \%$ & $20.0 \%$ \\
\hline N107 & Smallsrc & $\begin{array}{l}\text { Reduced size source, shrank to } 1 / 3 \text { size in MCNP } \\
\text { model based on Reference } 9 \text { using corrected } \\
\text { shim plate. }\end{array}$ & $66 \%$ & $4 \%$ & $6.1 \%$ \\
\hline N108 & Tophat & $\begin{array}{l}\text { Tophat thickness increased from } 0.166 \text { to } 0.477 \\
\mathrm{~cm} \text {. Baseline is from B-31 in Reference } 9 \text {, and } \\
\text { perturbation is from MCNP model's updated } \\
\text { Tophat. It is expected that any measured value is } \\
\text { roughly } 10 \% \text { of this difference. }\end{array}$ & $65 \%$ & $10 \%$ & $15.3 \%$ \\
\hline
\end{tabular}

In the previous section, uncertainties were inferred as the effect of a perturbation on a single measurement quantity. However, in this section, the uncertainties are inferred as the effect of a perturbation on both the treatment and control measurement quantities to credit the two-parameter design, which reduces the 
effects of biases in the results. Instead of evaluating the effect of the perturbation on the measured dose, the effect of the perturbation on the change of the dose due to the addition of $\mathrm{a} \mathrm{SiO}_{2}$ shield is used. Therefore, the uncertainty calculation is slightly different and is presented here. To calculate total uncertainty, the first step is to compute a transmission factor $\left(\tau_{i}\right)$ for a perturbation $i$ by dividing the treatment case $(T)$ by the control case $(C)$ tally:

$$
\tau_{i}=\frac{T_{i}}{C_{i}}
$$

Eq. (4) is the main difference from the preceding section, and therefore different subscripts are used for the quantities in this section. The absolute uncertainty $\left(\sigma_{\tau_{i}}\right)$ of $\tau_{i}$ from Monte Carlo (MC) uncertainty is computed as

$$
\sigma_{\tau_{i}}=\frac{T_{i}}{C_{i}} \sqrt{\left(\frac{\sigma_{T_{i}}}{T_{i}}\right)^{2}+\left(\frac{\sigma_{C_{i}}}{C_{i}}\right)^{2}}
$$

where $\sigma$ is the standard deviation of the quantity in the subscript. Eqs. (4) and (5) are also applied for the unperturbed case (subscript $u$ in the following equations).

To obtain the change in the attenuation factor introduced by the perturbation, Eq. (6) is used,

$$
R_{\tau_{i}}=S_{i}\left(\frac{\tau_{i}}{\tau_{u}}-1\right)
$$

where $S_{i}$ is the scaling factor for the perturbation. The absolute uncertainty for $R_{\tau_{i}}$ is computed as

$$
\sigma_{\tau_{i}}=S_{i} \frac{\tau_{i}}{\tau_{u}} \sqrt{\left(\frac{\sigma_{\tau_{i}}}{\tau_{i}}\right)^{2}+\left(\frac{\sigma_{\tau_{u}}}{\tau_{u}}\right)^{2}}
$$

The total uncertainty in the attenuation factor from all perturbations is then computed using a sum of squares:

$$
R_{\tau}=\sum_{i} R_{\tau_{i}}^{2}
$$

Overall, uncertainties of the responses to many perturbations were roughly on the order of the size of the response itself, so it is expected that uncertainties to these perturbations are lower than that stated in the tables below.

As an example of computation of relative difference in efficacy of a shielding sample due to a perturbation, the $R_{\tau_{i}}$ for the bottom_box_density case is computed in this paragraph. First $\tau_{u}$ for the unperturbed baseline case (N101) is computed as 0.6634 as per Eq. 4 by dividing the $\mathrm{SiO}_{2}$ treatment case tally of $3210 \mathrm{n} / \mathrm{cm}^{2}$ s by the unshielded control case tally of $4839 \mathrm{n} / \mathrm{cm}^{2} \mathrm{~s}$. Next, $\tau_{i}$ for the perturbed bottom_box_density case (N102) is computed as 0.6450 as per Eq. 4 by dividing the $\mathrm{SiO}_{2}$ treatment case tally of $3196 \mathrm{n} / \mathrm{cm}^{2} \mathrm{~s}$ by the unshielded control case tally $4955 \mathrm{n} / \mathrm{cm}^{2} \mathrm{~s}$. Then Eq. 6 is applied with a scaling factor $\left(S_{i}\right)$ of 0.5 to arrive at an $R_{\tau_{i}}$ of $-1.38 \%$. This requires 4 tallies per row, whereas Table 9 only uses 2 tallies per row. The physical interpretation of this is that the effect of the $\mathrm{SiO}_{2}$ shield is changed by $1.38 \%$ when the bottom box density is perturbed. 
Table 12. Changes in neutron transmission due to perturbations

\begin{tabular}{cccc}
\hline Case number & Perturbation & $\begin{array}{c}\text { Relative difference from } \\
\text { parameter uncertainty } \\
\left(R_{\tau_{i}}\right)\end{array}$ & $\begin{array}{c}\text { Absolute Monte Carlo } \\
\text { uncertainty }\left(\sigma_{\tau_{i}}\right)\end{array}$ \\
\hline N102 & bottom_box_density & $-1.38 \%$ & $1.22 \%$ \\
N103 & bottom_box_width & $-0.21 \%$ & $0.15 \%$ \\
N104 & bottom_plate & $-0.27 \%$ & $0.25 \%$ \\
N105 & lorho & $-0.02 \%$ & $0.24 \%$ \\
N106 & NoBandNoClamp & $0.06 \%$ & $0.56 \%$ \\
N107 & smallsrc & $0.15 \%$ & $0.16 \%$ \\
N108 & Tophat & $-0.35 \%$ & $0.38 \%$ \\
& & & \\
& Uncertainty from near-source \\
& perturbations $\left(R_{\tau}\right)$ & $1.47 \%$ & \\
\hline
\end{tabular}

Table 13. Changes in gamma transmission due to perturbations

\begin{tabular}{cccc}
\hline Case number & Perturbation & $\begin{array}{c}\text { Relative difference } \\
\text { from parameter } \\
\text { uncertainty }\left(R_{\tau_{i}}\right)\end{array}$ & $\begin{array}{c}\text { Absolute Monte Carlo } \\
\text { uncertainty }\left(\sigma_{\tau_{i}}\right)\end{array}$ \\
\hline N102 & bottom_box_density & $-0.28 \%$ & $0.77 \%$ \\
N103 & bottom_box_width & $0.03 \%$ & $0.11 \%$ \\
N104 & bottom_plate & $0.55 \%$ & $0.19 \%$ \\
N105 & lorho & $-0.48 \%$ & $0.30 \%$ \\
N106 & NoBandNoClamp & $0.06 \%$ & $0.34 \%$ \\
N107 & smallsrc & $-0.02 \%$ & $0.11 \%$ \\
N108 & Tophat & $0.49 \%$ & $0.22 \%$ \\
& & & \\
& Uncertainty from near-source & $0.92 \%$ & \\
& perturbations $\left(R_{\tau}\right)$ & & \\
\hline
\end{tabular}

\subsection{UNCERTAINTY OF SOURCE INTENSITY}

The source intensity changes slightly from one burst to the next. Ultimately the team does not hold a shared position on the reliability of the existing data for uncertainty in source intensity. Because the team does not agree on the reliability of any of these data, additional work is needed to quantify source intensity and reproducibility. The 3\% 1-sigma uncertainty developed in this section is assumed for the remainder of the report, but the team's de facto position is that this should be verified prior to initiation of substantial investment in execution of the IER.

This section presents some analysis of the uncertainty inherent in the temperature rise measurement, that was done preliminarily and assumed in the remainder of the report. Temperature rise results from the number of fissions, which, over a brief interval of time, are proportional to the reactivity inserted, which is also proportional to the reactor period. Showing the percent deviation of temperature rise for a measured reactor period from a correlation should provide an upper bound to the percent deviation of temperature rise from the number of fissions that occurred in the system. 
Godiva bursts from 2016 to 2021 with reactor periods of 36-49 $\mu$ s were selected, corresponding to temperature rises of near $70^{\circ} \mathrm{C}$. A trendline was established, and a $3 \%$ standard deviation was found in the data from the trendline. It appears that some hysteresis may be occurring with what could be multiple parallel lines of points, or a clear line of points exists with intermittent noise. In either case, the reason is unclear. Therefore, a $3 \%$ uncertainty is taken to be the degree of accuracy of the temperature rise proxy for source intensity.

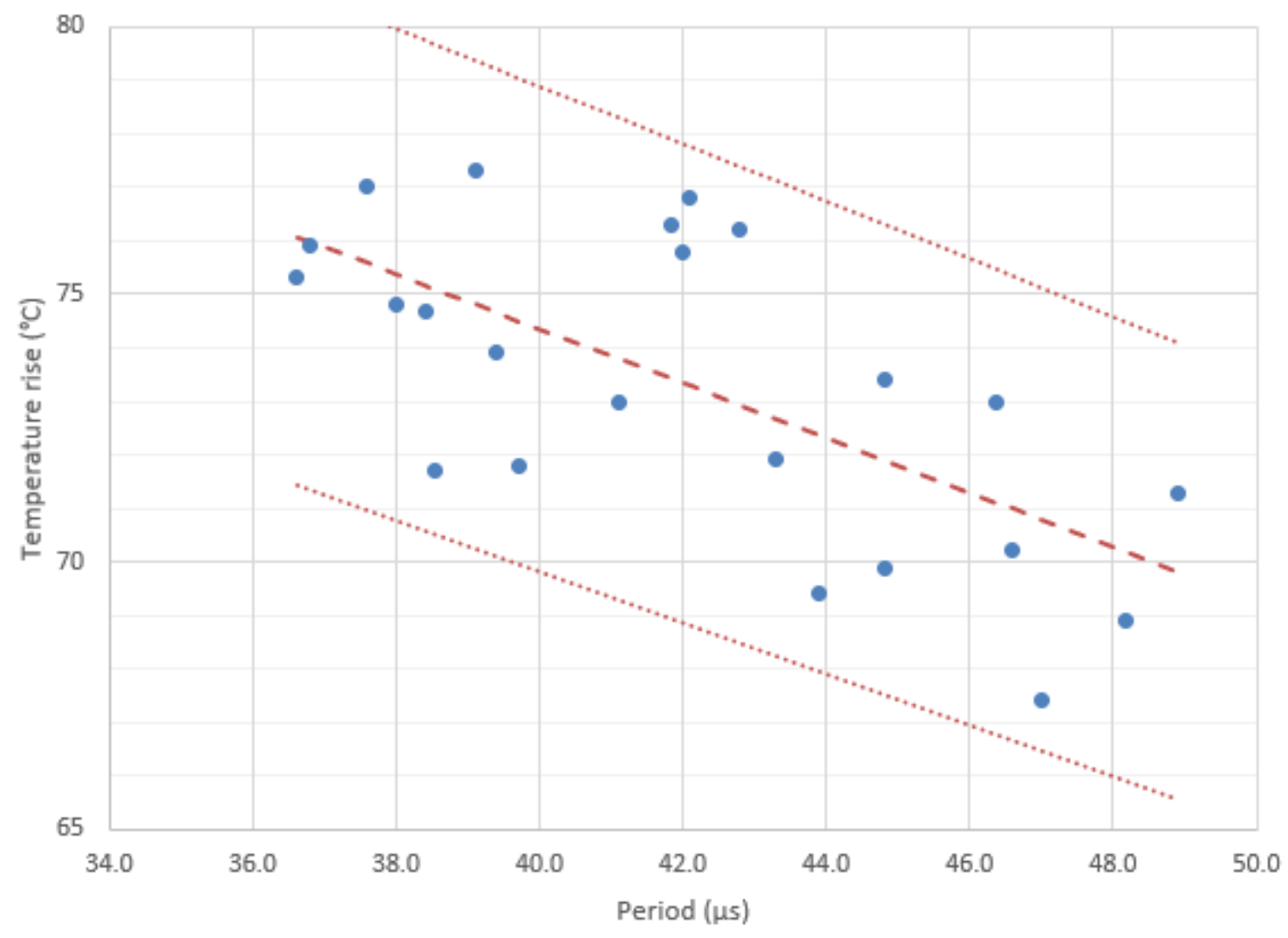

- Historic data $\quad$ - - Correlation $\quad . . . . . . .995 \% \mathrm{Cl}$ for correlation with $3 \%$ sigma

Figure 28. Correlation of temperature and reactor period.

An additional diagnostic for determining the absolute burst size is currently under development. While it is not relied upon in this report, this measurement technique may provide a decreased uncertainty in the burst size estimate compared to temperature rise. The new diagnostic will utilize the integral of the current measured with a scintillation detector operated in current mode during the burst. The detector will be composed of a Hamamatsu R12290U-53 phototube [29] and a 5.08 $\times 5.08 \mathrm{~cm}$ cylindrical EJ-228 fast plastic scintillator [30] read out by an oscilloscope. The diagnostic will rely on relating the integral of the current measured during a burst to activation foil measurements conducted for bursts of various sizes. Two sets of activation foil measurements will be performed: (1) measurements consisting of a wide range of burst sizes to determine the correlation between the integrated current measured by the detector during a burst and the absolute burst size, and (2) a series of measurements of bursts of similar size used to determine the precision of the diagnostic. The major sources of uncertainty are the uncertainty in the activation foil measurements ( $\sim 3 \%$ uncertainty or better can be obtained when using radiochemistry solution techniques) and the precision of the calibration of the scintillation detector (anticipated to be $<3 \%$ uncertainty). 
A key aspect of this diagnostic is that it will relate the relative integrated current to the burst size, not the absolute integrated current. The use of a relative scale will account for changes in the detector response (phototube and/or scintillator) caused by radiation damage. The relative scale will be determined using a calibration source (such as ${ }^{252} \mathrm{Cf}$ ) with low uncertainty in the emission. The current measured by the detector with the source placed at a fixed position will be used as the relative scale. The change in the detector response between measurements can be quantified using this calibration technique, so it can also be used to relate the integral of the measured current for different bursts over time.

Note that in the case of using a single source as the calibration standard, the uncertainty in the source emission does not factor into the uncertainty in the calibration. Uncertainty in the source emission only impacts the calibration uncertainty if multiple calibration sources are used. For example, if two different

${ }^{252} \mathrm{Cf}$ sources were used to calibrate the detector for two different bursts, then the detector efficiency and source emission must be precisely characterized, because the sources would be used to relate the two different calibrations. Therefore, they would add to the calibration uncertainty. A single calibration source standard will be used for IER-498 to avoid this increased uncertainty.

\subsection{TOTAL UNCERTAINTY}

The benchmark quantity combines counts from two measurements - a treatment and a baseline. Therefore, uncorrelated uncertainty would increase, but correlated uncertainties would tend to cancel. By definition, correlated uncertainties would produce similar differences in both tests, and because the benchmark quantity is a comparison between the tests, the differences would cancel. Because correlation is difficult to characterize, no correlation is assumed between the treatment and baseline measurements for many categories. This assumption results in a larger estimate of total uncertainty. The $3.3 \%$ far-fromsource and the $1.47 \%$ near-source geometric and density uncertainties result in a relative uncertainty of the benchmark quantity $Q$ of roughly $7.1 \%$ once other uncertainties are included. This is shown in Table 14.

Source and detector uncertainties are present in the experiment, in addition to the geometric uncertainty. In the SILENE experiment, detector uncertainties were on the order of 2-3\%. In the SILENE test, careful source measurement resulted in a source uncertainty of $5 \%$ because of the inability to better instrument the source. The two-measurement benchmark described in Section 2.5 is used with the treatment/baseline values for a given measurement as the benchmark parameter. The source for two measurements is instrumented with indirect measures such as temperature rise and burst period. It is expected that a 95\% uncertainty band of $+/-6 \%$ percent appears achievable from all the instrumentation on the assembly, which corresponds to a standard deviation of $3 \%$.

Table 14 estimates an expected uncertainty in the benchmark quantity (treatment measurement divided by baseline). Assuming the source strength proxy has a 3\% uncertainty, and a $2 \%$ detector uncertainty exists, as in many SILENE neutron foil measurements, a total benchmark quantity uncertainty of $6.91 \%$ (one sigma) is expected as based on the following discussion. This is similar to the SILENE uncertainties and is computed in Table 14. For convenience, it is assumed that no uncertainties in Table 14 are correlated, so they are assumed not to cancel in the computation of the benchmark quantity uncertainty. Table 14 propagates uncorrelated uncertainties (geometric, detector, source strength) to give relative uncertainty in source strength normalized measurement. It is calculated as described below.

Because geometric uncertainty and detector uncertainties are both relative to the measurement, the relative uncertainties can be combined with Pythagorean addition to get a total measurement uncertainty. Because this is then divided by the source strength proxy, this also propagates with Pythagorean addition, resulting in a $4.89 \%$ relative uncertainty. In the fifth row, the two $4.89 \%$ relative uncertainties are 
combined to be $6.91 \%$ again with Pythagorean addition. The $1.47 \%$ uncertainty in relative measurement near the source is then added using Pythagorean addition, for a total uncertainty of approximately $7 \%$.

Table 14. Hypothetical uncertainty for two measurement neutron benchmark quantity.

\begin{tabular}{rcc}
\hline & Baseline & Treatment \\
\hline Far-from-source geometric and density relative uncertainty in & $3.30 \%$ & $3.30 \%$ \\
measurement & $2.00 \%$ \\
Detector relative uncertainty in measurement & $2.00 \%$ & $3.00 \%$ \\
Relative uncertainty in source strength proxy & $3.00 \%$ & $4.89 \%$ \\
Relative uncertainty in source strength normalized measurement & $4.89 \%$ & $\mathbf{6 . 9 1 \%}$ \\
Relative uncertainty benchmark quantity subtotal & $1.47 \%$ \\
\hline Near-source geometric and density relative uncertainty in benchmark & $\mathbf{7 \%}$ \\
\hline
\end{tabular}

Table 15 shows a similar computation to Table 14, but for a single measurement case. Here, because of limitations in measurement of the absolute number of fissions occurring, a 5.2\% uncertainty in the source strength is assumed. The 5.2\% source strength uncertainty is the Pythagorean sum of (1) the $3.0 \%$ source strength proxy, (2) the $3 \%$ source strength proxy for a calibration measurement, and (3) a $1.53 \%$ uncertainty for the calibration $[31,32]$, which would require using a model of the Godiva assembly for the calibration. Notably, the single parameter benchmark could still neglect bias that may be present in the system, which would otherwise cancel in the two-parameter benchmark. Therefore, the two-parameter benchmark is preferred to that given in Table 15 .

Table 15. Hypothetical uncertainty for single measurement neutron benchmark quantity.

\begin{tabular}{rr}
\hline & $\begin{array}{c}\text { Nominal } \\
\text { uncertainty }\end{array}$ \\
\hline Far-from-source geometric and density relative uncertainty in measurement & $3.30 \%$ \\
Detector relative uncertainty in measurement & $2.00 \%$ \\
Relative uncertainty in source strength proxy & $5.20 \%$ \\
Near-source geometric and density relative uncertainty in benchmark quantity & $2.21 \%$ \\
\hline Total uncertainty & $\mathbf{7 \%}$ \\
\hline
\end{tabular}

For photons, the total uncertainty for the two-measurement benchmark quantity is on the order of $14 \%$ when calculated in a manner like that used for the two-measurement neutron benchmark uncertainty, and assuming a 5\% gamma measurement uncertainty typical of some TLDs. Details are provided in Table 16. Because gammas are secondary to neutron production, it is likely that the non-Monte Carlo code uncertainties are also larger than the neutron uncertainties. 
Table 16. Potential uncertainty for two measurement gamma benchmark quantity

\begin{tabular}{rrr}
\hline & Baseline & Treatment \\
\hline Far-from-source geometric and density relative uncertainty in measurement & $8.34 \%$ & $8.34 \%$ \\
Detector relative uncertainty in measurement & $5.00 \%$ & $5.00 \%$ \\
Relative uncertainty in source strength proxy & $3.00 \%$ & $3.00 \%$ \\
Relative uncertainty in source strength normalized measurement & $10.18 \%$ & $10.18 \%$ \\
\hline Relative uncertainty benchmark quantity subtotal & & $\mathbf{1 4 . 3 9 \%}$ \\
Near-source geometric and density relative uncertainty in benchmark & \\
quantity & $0.92 \%$ \\
\hline Total uncertainty & $\mathbf{1 4 \%}$ \\
\hline
\end{tabular}

\subsubsection{Summary}

A sensitivity study was performed on 26 cases. The Monte Carlo uncertainty dominated, but it is estimated that the total neutron uncertainty is approximately $7 \%$ for the benchmark quantity, and the total gamma uncertainty is approximately $14 \%$ using a two-parameter benchmark, supposing the supporting calculations are converged. Both uncertainties are acceptable.

\subsection{NEUTRON STREAMING}

Streaming of neutrons around the shielding sample is possible. In the case of the lead inner liner, the path length before a fast neutron is absorbed is rather long, so it is conceivable that using a lead inner liner in the room return shield surrounding the shield sample could result in neutron streaming around the sample. This is principally an issue for strongly attenuating samples such as polyethylene. In Figure 29, spectra at the detector location are plotted for various cases. The two curves at the bottom of the plot are measures of streaming for a room return shield having. They are fluxes at the measurement location for simulated opaque shielding samples of 5 and $14 \mathrm{~cm}$ made from ultra-dense $\mathrm{SiO}_{2}$. No radiation can traverse these ultra-dense samples, so the only radiation that can be detected must stream around them. Thus, predictions for opaque shielding samples are used to provide a reasonable measure of the streaming around the shielding samples. In Figure 29, only the blue $15.1 \mathrm{~cm}$ polyethylene curve has values approaching that of the curve resulting from streaming around an opaque shielding sample. Presumably, minor streaming around the shielding sample would be accounted for in the benchmark simulations. The main issue of concern is whether the streaming dominates a measurement. The worst case evaluated was a thick polyethylene sample, which is shown in Figure 30. The streaming around the opaque shielding sample is a very large fraction of the flux at the detector in the energy range from approximately 1 to 500 $\mathrm{keV}$. 


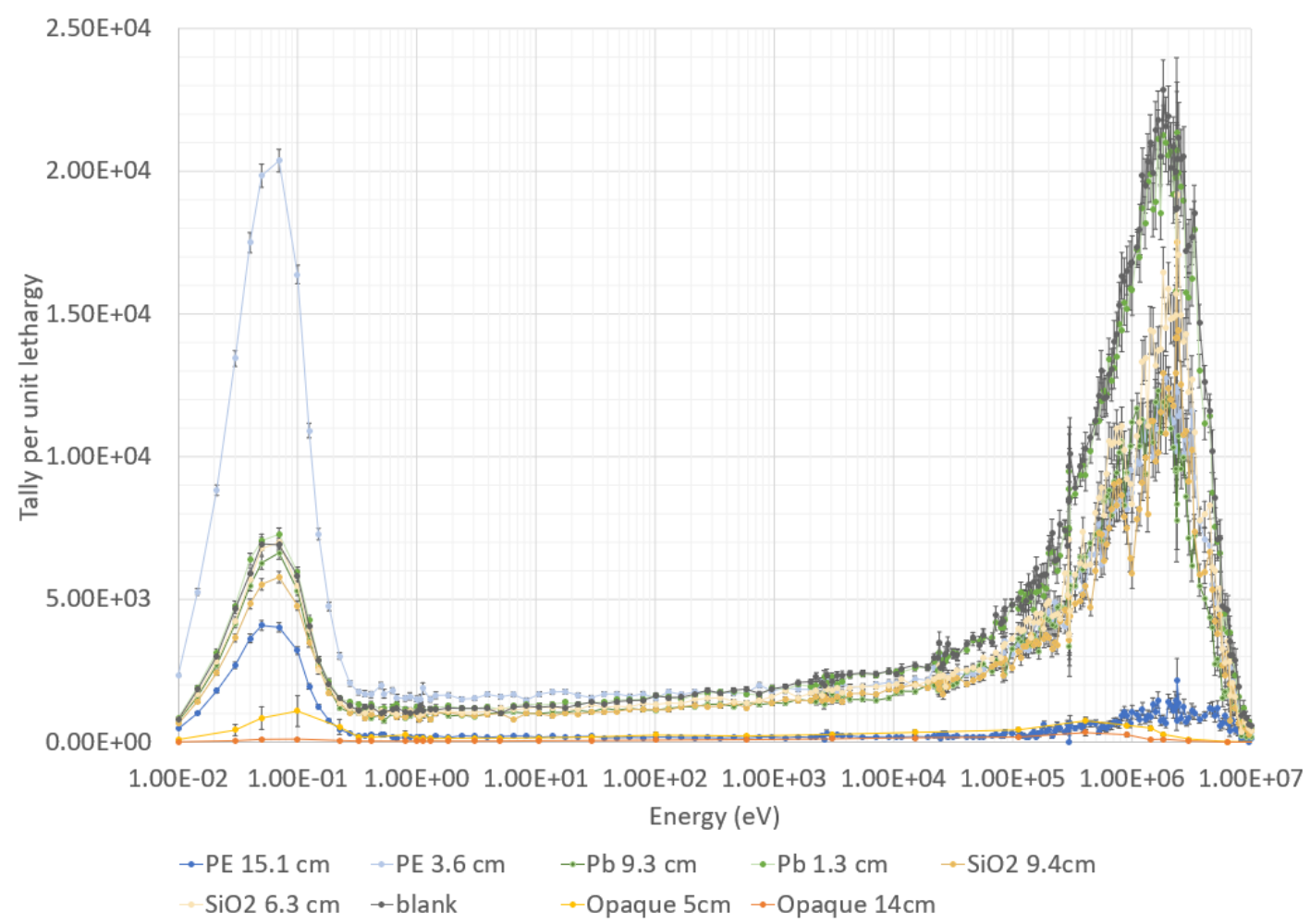

Figure 29. Detector fluxes of simulated opaque shielding samples with no flux trap (orange and yellow at bottom of graph) vs. fluxes for ordinary shielding samples also with no flux trap (other colors).

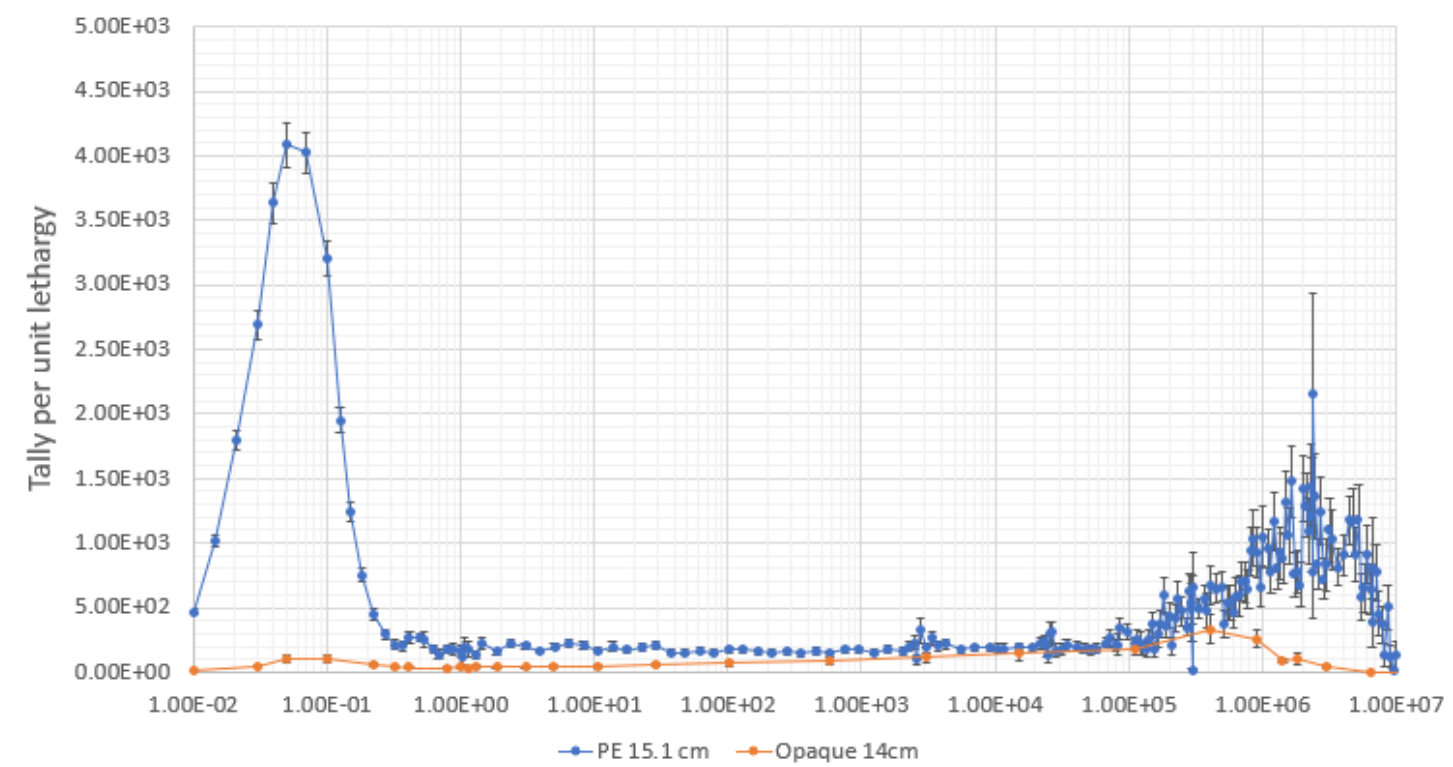

Figure 30. Detector fluxes of simulated opaque shielding sample with no flux trap (orange at bottom of graph) vs fluxes for polyethylene shielding sample (blue).

The primary case in which the streaming impacts results is for fast neutron transmission through a thick polyethylene shield sample. It may be prudent to design flexibility to allow for placement of the flux trap in the room return shield in case highly moderating or highly attenuating samples will be evaluated in future tests. 


\section{SCHEDULE BUDGET AND STAFFING}

\subsection{TEST MATRIX}

From the detector ranges, it seems that the experiment matrix in Table 17 is reasonable. The steady state foils would be exposed in two batches: one batch (group A) that is not time sensitive and another batch (group B) that requires counting within hours after exposure. The non-electronic, passive detectors used will be the SNAP, PNAD, TLD, RPL glass, and silicon diode detectors. Nominally three of each detector will be included to obtain statistics and error control. Group B is expected to include, the SNAP, and PNAD. The TLDs, RPL glass, and silicon diodes would make up group A.

Table 17. Test matrix.

\begin{tabular}{lrcr}
\hline Shielding & \multicolumn{3}{c}{ Number of Detectors } \\
\cline { 2 - 5 } Sample & $\begin{array}{c}\text { Steady state } \\
\text { operation } \\
\text { electronic } \\
\text { detectors }\end{array}$ & $\begin{array}{c}\text { Steady state } \\
\text { operation passive } \\
\text { detectors }\end{array}$ & $\begin{array}{c}\text { Burst } \\
\text { operation } \\
\text { passive } \\
\text { detectors }\end{array}$ \\
\hline Blank & $2+$ & 2 & 2 \\
$\mathbf{P b ~ 1 0 c m}$ & 1 & 1 & 2 \\
$\mathbf{P b ~ 5 c m}$ & 1 & 1 & 2 \\
$\mathbf{P E ~ 1 0 c m ~}$ & 1 & 1 & 2 \\
$\mathbf{P E ~ 5 c m ~}$ & 1 & 1 & 2 \\
$\mathbf{S i O}_{\mathbf{2}} \mathbf{1 0 c m}$ & 1 & 1 & 2 \\
$\mathbf{S i O}_{\mathbf{2}} \mathbf{5 c m}$ & 1 & 1 & 2 \\
total & $8+$ & 8 & 14 \\
\hline
\end{tabular}

If time allows, additional shielding samples can be tested. These samples include graphite, sodium chloride, and iron.

\subsection{TEST SCHEDULE}

The test schedule will occur over the course of three weeks, with the first week being followed by a regroup period of two to three weeks. The spacing of tests will allow time to address any issues encountered in the first week of testing. Given that this is an entirely new test, there is increased risk that a measurement will not be successfully performed. Adding some time to remediate the issues will reduce the consequences of such an occurrence.

Week 1 will consist of getting baseline measurement with every testing configuration. The room return shield will be placed and aligned with the source. The electronic detectors will be installed and calibrated as needed. Each detector will be tested by itself, and then all three will be tested together.

Following calibration, the first measurement to be made using the room return shield will be a blank measurement (no shielding sample installed in the room return shield). The power of the Godiva IV assembly will be increased until a sufficient reading is obtained at the medium-high end of the detectors' count rates. This will help avoid pile-up but will have a sufficient count rate so that information can be obtained on some of the less common higher energies. 
Next, passive detectors (PNAD, RPL glass, $\mathrm{CaF}_{2} \mathrm{TLD}$ ) will be installed, again with a blank shielding sample. The assembly will be powered at $10 \mathrm{~W}$ ( $44 \mathrm{nA}$ on the intermediate power detector system) for one hour. Two batches of detectors will be used. The first batch will be less time sensitive, so they can be read without urgency. The second set of detectors will include foils made of materials such as indium, with shorter half-lives, so these would need to be read immediately after exposure. First, the detectors that are not time sensitive will be tested, and then the detectors that are time sensitive will be tested. Then, both types of detectors will be sent to the NAD lab for counting, with the indium being counted first because of its short half-life. The results can be used to scale exposures for future tests.

At the end of the first week, burst tests will be performed: once with the time-insensitive detectors, and again with the time-sensitive detectors. Bursts are expected to occur at $70^{\circ} \mathrm{C}$, but based on initial results, this may be modified.

After a two- or three-week pause, tests with shielding samples will commence. Tests associated with equipment (electronic or passive) that has issues in the first week may be modified, postponed, canceled, or replaced with more promising tests. That is the impetus of performing one of each type of test first.

Table 18 below lists the time estimates and staff needed for each activity that will be performed during the experiment. The times for the Godiva IV facility usage are shown. The staff codes are keyed out in Table 19.

The remainder of the schedule is outlined in Table 20. The first half of week two will consist of tests with electronic detectors, followed by tests with steady-state configuration. Passive detectors will be used, and every shielding sample will be in the steady-state configuration. Sets of detectors will be alternated with shielding samples to obtain the most necessary data and to save time. Finally, week three will include burst tests.

Table 21 lists rough-order-of-magnitude costs for apparatus acquisition. Actual costs may vary. 
Table 18. Test work breakdown

\begin{tabular}{|c|c|c|c|}
\hline \multirow[t]{2}{*}{ Task } & \multicolumn{2}{|c|}{$\begin{array}{l}\text { Task times } \\
\text { (hours) }\end{array}$} & \multirow{2}{*}{$\begin{array}{c}\text { Staff } \\
\text { needed }\end{array}$} \\
\hline & Low & High & \\
\hline Align RRS with source & 1 & 3 & RG \\
\hline Install detectors (1 day) & 3 & 12 & EBTG \\
\hline Place neutron electronics, verify counting & & & E \\
\hline Place photon electronics, verify functionality & & & B \\
\hline Place thermal neutron electronics, verify functionality & & & $\mathrm{T}$ \\
\hline First test & 0.9 & 3 & EBTG \\
\hline Place driver source, and increase power until detector readings are sufficient & 0.5 & 1 & \\
\hline Remove detectors & 0.3 & 1 & \\
\hline Compare to expectations to determine if things are functioning correctly & 0.1 & 1 & \\
\hline Passive detector test & 1.8 & 3.2 & \\
\hline Place passive detectors & 0.3 & 1 & PCG \\
\hline Run $10 \mathrm{~W}$ for an hour & 1.2 & 1.2 & G \\
\hline Remove detectors & 0.3 & 1 & PCG \\
\hline Process at count lab & & & PCG \\
\hline Place detectors and electronics & 0.5 & 3 & EBTG \\
\hline Electronic detector tests & 0.55 & 1.45 & EBTG \\
\hline Run at low power to record counts. & 0.25 & 0.25 & EBTG \\
\hline Check vs expectation and previous measurement & 0.1 & 0.2 & EBTG \\
\hline Place first shield sample and repeat & 0.2 & 1 & EBTG \\
\hline Passive detector test & 1.6 & 3.2 & PGC \\
\hline Place passive detectors (and pull bubble detectors) & 0.2 & 1 & PGC \\
\hline \multicolumn{4}{|l|}{$\begin{array}{l}\text { Compare bubble to expectations. Does it appear source intensity may need } \\
\text { scaled? }\end{array}$} \\
\hline Run $10 \mathrm{~W}$ for an hour (or amount determined from previous tests) & 1.2 & 1.2 & PG \\
\hline Remove detectors & 0.2 & 1 & PGC \\
\hline Process at count lab & & & PC \\
\hline Burst tests & 1.8 & 3 & \\
\hline Place passive detectors & 0.3 & 1 & PGC \\
\hline Burst & 1 & 1 & G \\
\hline Room cooldown & 0.5 & 1 & G \\
\hline Remove detectors while placing next set of passive detectors & 0.3 & 1 & PGC \\
\hline Process at count lab & & & PC \\
\hline
\end{tabular}


Table 19. Specialist codes for work breakdown

\begin{tabular}{l|l}
\hline \multicolumn{1}{c}{ CODE } & \multicolumn{1}{c}{ SPECIALIST } \\
\hline $\mathbf{E}$ & EJ-309 specialist \\
$\mathbf{B}$ & BGO specialist \\
$\mathbf{T}$ & Thermal detector specialist \\
$\mathbf{P}$ & Passive detector Specialist \\
$\mathbf{C}$ & Count lab specialist \\
$\mathbf{G}$ & Godiva Specialists \\
$\mathbf{R}$ & RRS specialist \\
\hline
\end{tabular}

Table 20. Detailed schedule (orange rows indicate trip to count lab)

\begin{tabular}{|c|c|c|c|c|}
\hline & Day & Duration (h) & Task & Sample \\
\hline \multirow{10}{*}{$\begin{array}{l}\bar{v} \\
\frac{\pi}{8} \\
\overrightarrow{3}\end{array}$} & $\mathrm{M}$ & 4 & \multicolumn{2}{|l|}{ Place RRS, and unpack, get onsite } \\
\hline & M & 1.5 & Passive detector test $\mathrm{A}$ & Blank \\
\hline & $\mathrm{M}$ & 1.5 & Passive detector test B & Blank \\
\hline & $\mathrm{Tu}$ & 3 & Electronic detector A & Blank \\
\hline & $\mathrm{Tu}$ & 2.5 & Electronic detector B & Blank \\
\hline & $\mathrm{Tu}$ & 2.5 & Electronic detector $\mathrm{C}$ & Blank \\
\hline & $\mathrm{W}$ & 3 & Electronic detectors A, B, C & Blank \\
\hline & $\mathrm{W}$ & 2 & Burst test detector set A & Blank \\
\hline & Th & 2 & Burst test detector set B & Blank \\
\hline & Th & 3 & Burst test detector set B & $\mathrm{SiO}_{2} 10 \mathrm{~cm}$ \\
\hline \multirow{13}{*}{$\frac{N}{\frac{v}{d}} \frac{d}{3}$} & $\mathrm{M}$ & 2 & Passive detector test A & $\mathrm{Pb} 5 \mathrm{~cm}$ \\
\hline & $\mathrm{M}$ & 2 & Passive detector test B & $\mathrm{Pb} 10 \mathrm{~cm}$ \\
\hline & M & 2 & Passive detector test A & PE $10 \mathrm{~cm}$ \\
\hline & $\mathrm{Tu}$ & 2 & Passive detector test B & PE 5 \\
\hline & $\mathrm{Tu}$ & 2 & Passive detector test $\mathrm{A}$ & $\mathrm{SiO}_{2} 5 \mathrm{~cm}$ \\
\hline & $\mathrm{Tu}$ & 2 & Passive detector test B & $\mathrm{SiO}_{2} 10 \mathrm{~cm}$ \\
\hline & $\mathrm{Tu}-\mathrm{W}$ & 3 & Electronic & $\mathrm{Pb} 5 \mathrm{~cm}$ \\
\hline & $\mathrm{W}$ & 1 & Electronic & $\mathrm{Pb} 10 \mathrm{~cm}$ \\
\hline & $\mathrm{W}$ & 1 & Electronic & PE $5 \mathrm{~cm}$ \\
\hline & $\mathrm{W}$ & 1 & Electronic & PE $10 \mathrm{~cm}$ \\
\hline & $\mathrm{W}$ & 1 & Electronic & $\mathrm{SiO}_{2} 5 \mathrm{~cm}$ \\
\hline & $\mathrm{W}$ & 3 & Electronic & $\mathrm{SiO}_{2} 10 \mathrm{~cm}$ \\
\hline & Th & 3 & Burst test detector set B & $\mathrm{Pb} 5 \mathrm{~cm}$ \\
\hline \multirow{10}{*}{$\frac{m}{\frac{\pi}{d}}$} & $\mathrm{M}$ & 3 & Burst test detector set A & $\mathrm{Pb} 5 \mathrm{~cm}$ \\
\hline & $\mathrm{M}$ & 3 & Burst test detector set B & $\mathrm{Pb} 10 \mathrm{~cm}$ \\
\hline & $\mathrm{Tu}$ & 3 & Burst test detector set A & $\mathrm{Pb} 10 \mathrm{~cm}$ \\
\hline & $\mathrm{Tu}$ & 3 & Burst test detector set A & $\mathrm{PE} 5 \mathrm{~cm}$ \\
\hline & $\mathrm{Tu}$ & 3 & Burst test detector set B & PE $5 \mathrm{~cm}$ \\
\hline & $\mathrm{W}$ & 3 & Burst test detector set A & PE $10 \mathrm{~cm}$ \\
\hline & $\mathrm{W}$ & 3 & Burst test detector set B & $\mathrm{PE} 10 \mathrm{~cm}$ \\
\hline & $\mathrm{W}$ & 3 & Burst test detector set A & $\mathrm{SiO}_{2} 5 \mathrm{~cm}$ \\
\hline & Th & 3 & Burst test detector set A & $\mathrm{SiO}_{2} 10 \mathrm{~cm}$ \\
\hline & Th & 3 & Burst test detector set B & $\mathrm{SiO}_{2} 5 \mathrm{~cm}$ \\
\hline
\end{tabular}


Table 21. Rough, order of magnitude cost and acquisition time estimates

\begin{tabular}{|c|c|c|c|c|c|c|c|c|c|c|}
\hline \multicolumn{2}{|c|}{ Number } & \multirow{2}{*}{ Measurement Apparatus } & \multicolumn{3}{|c|}{ Costs } & \multicolumn{5}{|c|}{ Task times (months) } \\
\hline 1 & & & $\$ 400 \mathrm{k}$ & & & 13 & & & & \\
\hline $\mathrm{a}$ & & Room return shield & $\$ 230 \mathrm{k}$ & & & & 12 & & & \\
\hline $\mathrm{i}$ & & Stand & & $\$ 114 k$ & & & & 9.5 & & \\
\hline & 1 & $\begin{array}{l}\text { Post-CED-2 engineering development (frame } \\
\text { structure) }\end{array}$ & & & $\$ 60 \mathrm{k}$ & & & & 4 & \\
\hline & 2 & Design acceptance/approval & & & $\$ 1 \mathrm{k}$ & & & & 0. & \\
\hline & 3 & Vendor selection & & & $\$ 1 \mathrm{k}$ & & & & 0. & \\
\hline & 4 & Buildout & & & $\$ 50 \mathrm{k}$ & & & & 4 & \\
\hline & 6 & Delivery & & & $\$ 2 \mathrm{k}$ & & & & 0. & \\
\hline ii & & Shield & & $\$ 116 \mathrm{k}$ & & & & 12 & & \\
\hline & 1 & Post-CED-2 Engineering development & & & $\$ 20 \mathrm{k}$ & & & & 4 & \\
\hline & 2 & Design acceptance/approval & & & $\$ 1 \mathrm{k}$ & & & & 0. & \\
\hline & 3 & Vendor selection & & & $\$ 1 \mathrm{k}$ & & & & 0. & \\
\hline & 4 & Materials & & & $\$ 40 \mathrm{k}$ & & & & 0 & \\
\hline & & Lead portion & & & & & & & & \\
\hline & & Polyethylene portion & & & & & & & & \\
\hline & 5 & Buildout & & & $\$ 50 \mathrm{k}$ & & & & 6 & \\
\hline & & Lead portion & & & & & & & & \\
\hline & & Polyethylene portion & & & & & & & & \\
\hline & 7 & Delivery & & & $\$ 4 \mathrm{k}$ & & & & 1 & \\
\hline & & & & & & & & & & \\
\hline $\mathrm{b}$ & & Data acquisition system & $\$ 75 \mathrm{k}$ & & & & 3 & & & \\
\hline $\mathrm{i}$ & & Detectors (setup and acquisition) & & $\$ 50 \mathrm{k}$ & & & & 3 & & \\
\hline & & EJ-309 detector & & & $\$ 10 \mathrm{k}$ & & & & & \\
\hline & & BGO detector & & & $\$ 10 \mathrm{k}$ & & & & & \\
\hline & & Thermal detector & & & $\$ 10 \mathrm{k}$ & & & & & \\
\hline & & PNAD detector & & & $\$ 3 \mathrm{k}$ & & & & & \\
\hline & & SWX set & & & $\$ 3 \mathrm{k}$ & & & & & \\
\hline & & SNAP set & & & $\$ 3 \mathrm{k}$ & & & & & \\
\hline & & RPL set & & & $\$ 3 \mathrm{k}$ & & & & & \\
\hline & & TLD set & & & $\$ 3 k$ & & & & & \\
\hline & & Mountings & & & $\$ 5 \mathrm{k}$ & & & & & \\
\hline ii & & Site acceptance/approval & & $\$ 5 \mathrm{k}$ & & & & 1 & & \\
\hline iv & & Testing? & & $\$ 20 \mathrm{k}$ & & & & 2 & & \\
\hline & & & & & & & & & & \\
\hline $\mathrm{c}$ & & Common elements & $\$ 70 \mathrm{k}$ & & & & 5 & & & \\
\hline $\mathrm{i}$ & & $\begin{array}{l}\text { Acceptability of overall approach and setup by } \\
\text { NCERC }\end{array}$ & & $\$ 10 \mathrm{k}$ & & & & 2 & & \\
\hline ii & & Procedure refinements & & $\$ 20 \mathrm{k}$ & & & & 2 & & \\
\hline iii & & Installation & & $\$ 10 \mathrm{k}$ & & & & 0.5 & & \\
\hline iv & & $\begin{array}{l}\text { Qualification (does everything meet what we } \\
\text { asked for) }\end{array}$ & & $\$ 30 \mathrm{k}$ & & & & 0.5 & & \\
\hline $\mathrm{d}$ & & Shielding samples ( $\$ 0.5 \mathrm{k}$ per sample) & $\$ 25 \mathrm{k}$ & & & & 1 & & & \\
\hline
\end{tabular}




\section{POTENTIAL SAFETY AND OTHER HAZARDS}

1. Compressed gasses in detectors in room return shield

Mitigation: make the shield sample larger than the bore; the closure could open at a lower pressure than the shield sample "failure." A calculation is still needed for this condition.

Hydrogen/methane/helium recoil detectors should be avoided since they use extreme pressures.

2. Mass of room return shield (handling/lifting)

Mitigation: use a room return shield frame to transport and tilt.

3. Handling shield sample (fingers)

Mitigation: fabricate the shielding sample out of smaller layers that are easier to lift.

4. Lifting / crush risk of clamshell

Mitigation: for lifting, consider screw jacks, or something not improvised, and reasonably fail safe.

5. Source overdosing electronic detectors through high intensity operation

Mitigation: in CED-3A, determine electronics that should be removed.

6. Lead contamination

Mitigation: powder coat the lead.

7. Fire loading (due to the tons of polyethylene)

Mitigation: check final mass of polyethylene, etc., against limits

8. Ability to decontaminate room return shield to allow removal from the room

Mitigation: during fabrication, coat, or seal to prevent internal surfaces that are not accessible from being exposed to dust/contamination. 


\section{CONCLUSION}

A workable design to perform a CAAS benchmark experiment is detailed herein. Key dimensions, materials, source intensity levels, and detectors are listed in this report. Sensitivity to 21 perturbations was determined to be acceptable. The next step will be for NCSP management to determine whether procurement should occur and if the experiment should proceed. The perturbation study suggests that the room return shield cavity radius and runout should be maintained to within a millimeter, the room return shield should be positioned carefully (perhaps with a laser range finder), and that the detectors should be mounted in a lightweight fixture such as aluminum, so their positioning is assured. Using a 3D scanner or photogrammetry to record part shapes may be beneficial. Further work is also needed to verify source reproducibility.

The experimental hardware has been designed in sufficient detail to support procurement of actual components. The overall room return shield geometry is defined, and methods for building the shapes are suggested but not specified. This remains unresolved so that the CED-3A team can negotiate changes with vendors. The mechanical design is at the concept stage, and the components have yet to be sized. The CED-3A team may select a vendor versed in steel framing to produce a frame to meet the requirements.

The detectors for the experiment have been selected and will be supplied by LANL, LLNL, and IRSN. An EJ-309-based neutron detector that directly detects fast neutrons will provide fast neutron data, as well as some gamma spectroscopic data. A second gamma detector based on the BGO scintillator will be provided by IRSN and is also expected to capture gamma spectra. A third ${ }_{3} \mathrm{He}$-filled SP9 thermal neutron detector can provide insight into the level of neutrons that have slowed down while traversing the shielding sample. PNADs will be used as integral detectors for a variety of energies and will also be used to instrument bursts and other measurements.

The source ranges necessary to produce reasonable detector responses have been calculated. These orderof-magnitude calculations were performed to determine the source strengths that should be used with each detector to produce reasonable measurements. This information allowed for development of the threeweek experimental plan. While the procurement plan from CED-1 is largely unchanged, detector procurement is likely to be more straightforward because existing systems can be employed.

The sensitivity study showed that the geometric, material density, and source uncertainty for each measurement were approximately $15 \%$ for gammas and $7 \%$ for neutrons, assuming a $3 \%$ uncertainty in source intensity. Ultimately the purpose of uncertainty evaluation here was to show feasibility and important design considerations within schedule and budget constraints of CED-2. Ultimately these uncertainties may increase or decrease with more detailed evaluations using real data in CED-4. Many uncertainties are artificially high due to limitations in Monte Carlo convergence. In the future, this could be addressed with larger perturbations, longer run times, or by fully adopting the method used in evaluation of uncertainties near the source. Additional cases were also examined to determine if a flux trap would be required; these tests showed that thicker samples of polyethylene or other strongly interacting materials could suffer from leakage around the shielding sample.

Further work is needed for the team to fully support going ahead with this IER. Specifically, the Godiva IV source intensity should be verified to be either reproducible or recordable with uncertainty sufficiently small to support the benchmark. Nominally a 5\% one sigma uncertainty in source intensity should be sufficient, but this value could change. Sufficient study likely requires some type of experimental work in a separate IER. 


\section{REFERENCES}

1. National Criticality Safety Program, Critical \& Subcritical Experiment Design Team Process Manual of the United States Department of Energy Nuclear Criticality Safety Program, Revision 1, 2016.

2. IEEE Standard for Software Verification and Validation Working Group, IEEE 1012-2016 - IEEE Standard for System, Software, and Hardware Verification and Validation, 2016.

3. I. Kodeli, E. Sartori, and B. Kirk, SINBAD Shielding Benchmark Experiments Status and Planned Activities, The American Nuclear Society's 14th Biennial Topical Meeting of the Radiation Protection and Shielding Division, Carlsbad New Mexico, USA. April 3-6, 2006.

4. T. M. Miller, C. Celik, K. L. McMahan, et al., Neutron Activation Foil and Thermoluminescent Dosimeter Responses to a Polyethylene Reflected Pulse of the CEA Valduc SILENE Critical Assembly, Oak Ridge National Laboratory, CEA Saclay, CEA Valduc, Y-12 NEA/NSC/DOC(95)03/VIII, ALARM-TRAN-CH2-SHIELD-001, ORNL/TM-2016/317, 2016.

5. T. M. Miller, C. Celik, K. L. McMahan, et al., Neutron Activation Foil and Thermoluminescent Dosimeter Responses to a Bare Pulse of the CEA Valduc SILENE Critical Assembly, Oak Ridge National Laboratory, CEA Saclay, CEA Valduc, Y-12 NEA/NSC/DOC(95)03/VIII, ALARM-TRANAIR-SHIELD-001, ORNL/TM-2015/462 Rev. 1, 2016.

6. T. M. Miller, C. Celik, K. L. McMahan, et al., Neutron Activation Foil and Thermoluminescent Dosimeter Responses to a Lead Reflected Pulse of the CEA Valduc SILENE Critical Assembly, Oak Ridge National Laboratory, CEA Saclay, CEA Valduc, Y-12 NEA/NSC/DOC(95)03/VIII, ALARMTRAN-PB-SHIELD-001, ORNL/TM-2016/316, 2016.

7. X. Knemp, Documentary summary of CRAC and SILENE experiments, IRSN/2019-00331, 2019.

8. Committee to Assess Health Risks from Exposure to Low Levels of Ionizing Radiation, Board on Radiation Effects Research, Division on Earth and Life Studies, Health Risks from Exposure to Low Levels of Ionizing Radiation: BEIR VII Phase 2, Washington DC, 2006.

9. R. D. Mosteller, "Godiva-IV Delayed-Critical Experiments and Description of an Associated PromptBurst Experiment," Los Alamos National Laboratory, NEA/NSC/DOC/(95)03/II, HEU-MET-FAST086, 2014.

10. ePlastics, “1.000" X 48" X 96" 5\% BORATED PE,” accessed March 4, 2021, https://www.eplastics.com/sheets/borated-polyethylene/

11. R. A. Weldon, Jr, T. E. Cutler, et al. Rossi Alpha Measurements - Rapid Organic (n, $\gamma$ ) Discrimination Detector (RAM-RODD) system capabilities Unpublished Draft.

12. F. Pino et al., "The Light Output and the Detection Efficiency of the Liquid Scintillator EJ-309." Applied Radiation and Isotopes 89, pp. 79-84, 2014.

13. W. A. Wieselquist, R. A. Lefebvre, and M. A. Jessee, Eds., SCALE Code System, ORNL/TM2005/39, Oak Ridge National Laboratory, Oak Ridge, TN, Version 6.2.4, 2020.

14. GADRAS D. J. Mitchell et al., "GADRAS Detector Response Function," SAND2014-19465, Sandia National Laboratories (2014)

15. D. Heinrichs et al., Final Design for an International Intercomparison Exercise for Nuclear Accident Dosimetry at the DAF Using GODIVA-IV, LLNL-TR-661851, 2014.

16. Mirion Technologies, "Falcon 5000 Portable HPGe-Based Radionuclide Identifier," Brochure, 2017.

17. Mirion Technologies, "iSolo Alpha/Beta Counting System” Brochure, 2014. 
18. Institut de Radioprotection et de Sûreté Nucléaire "Dosimétrie de criticité," Brochure accessed March 2021, http://dosimetrie.irsn.fr/fr-fr/prestations/dosimetrie-passive/criticite

19. Shieldwerx, "SWX-1550 Series Neutron Activation Foils," Brochure, 2018.

20. D. Hickman et al., "Dosimetry Characterization of the Godiva Reactor Under Burst Conditions," LLNL-TR-729408, 2017.

21. P. J. Griffin, SNL-SAND-IV v. 0.9 (beta). Computer software, version 00. US DOE, Oct. 5, 2016, https://www.osti.gov/biblio/1344302-snl-sand-iv-beta.

22. F. Vanhavere et al., "The Life Span of the BD-PND Bubble Detector," Radiation Protection Dosimetry, 85:1-4, pp. 27-30, 1999.

23. B. J. Lewis et al., "Review of Bubble Detector Response Characteristics and Results from Space," Radiation Protection Dosimetry, 150:1, pp. 1-21, 2012.

24. J. E. Partridge et al., "A Comparison of CaF2:Mn and LiF Thermoluminescent Dosimeters for Environmental Radiation Monitoring,” EPA 520/5-73-006, 1973.

25. K. R. DePriest, "Neutron Contribution to CaF2:Mn Thermoluminescent Dosimeter Response in Mixed (n/y) Field Environments," SAND2002-3778, 2002.

26. L. M. Choate and T. R. Schmidt, "New Neutron Simulation Capabilities Provided by the Sandia Pulse Reactor (SPR-III) and the Upgraded Annular Core Pulse Reactor (ACPR)," IEEE Radiation Effects Conference, Albuquerque, NM, 1978, https://www.osti.gov/servlets/purl/6607485.

27. Bubble Technology Industries, "Bubble Detectors: Neutron Dosimeters” Brochure, May 7, 2009.

28. Personal communication with Francois Trompier at IRSN September 24, 2020.

29. Hamamatsu, "Ultra-fast Photodetector Biplanar Phototubes: R12290U Series, R1328U Series," Datasheet, 2020, https://www.hamamatsu.com/resources/pdf/etd/R12290U_R1328U_TPT1028E.pdf

30. Eljen Technology, "Fast Timing Plastic Scintillator EJ-228, EJ-230,” Datasheet, 2021,

31. D. Pierson et al., "Improved Cumulative Fission Yield Measurements with Fission Spectrum Neutrons on 235U," Nuclear Data Sheets, vol. 155, pp. 86-97, Jan. 2019, doi: 10.1016/j.nds.2019.01.005.

32. B. D. Pierson et al., "Improved Cumulative Fission Yield Measurements with Fission Spectrum Neutrons on 238U," Nuclear Data Sheets, vol. 163, pp. 249-260, Jan. 2020, doi:

10.1016/j.nds.2019.12.006. 


\section{APPENDIX A. SHADOW CONE}

\section{A.1 SHADOW CONE UNCERTAINTY MODEL}

The detector response measurement taken without a shadow cone $(D)$ is includes several values:

$d$, the "true underlying value" of the measured response,

$d \epsilon_{s}$, the random error of the source on the measurement (scales linearly with measurement value, described by standard deviation $\sigma_{s}$ ),

$\epsilon_{d}$, the other random error of a given measurement (described by standard deviation $\sigma_{m}$, and

$d \beta_{d}$, a non-random "error" in the measured response that scales with response value.

The detector response measurement without a shadow cone $(D)$ can thus be broken up into components, as follows:

$$
D=d+\epsilon_{d}+d \epsilon_{s}+d \beta_{d}
$$

The detector response, a measurement of room return with a shadow cone $(R)$, is includes several values:

$r$, the "true underlying value" of the shadowed measurement,

$r \epsilon_{s}$, the random error of the source on the shadowed measurement (scales linearly with measurement value, described by standard deviation $\sigma_{s}$ ),

$\epsilon_{r}$, the other random error of a given shadowed measurement (described by standard deviation $\sigma_{r}$, $r \beta_{r t}$, a non-random "bias" form radiation leaking through the shadow cone (proportional to underlying dose rate times some unit error term), and

$r \beta_{r}$, the other nonrandom "bias" in the measured response (scales with response value).

The detector response, a measurement of room return with a shadow cone $(R)$, can be broken into components as follows,

$$
R=r+\epsilon_{r}+r \epsilon_{s}+r \beta_{r}+r \beta_{r t} .
$$

$\mathrm{C}$ is the calculated room return free value. It is computed as

$$
C=D-R \text {. }
$$

$\mathrm{c}$ is the underlying room return free value. It is computed as

$$
c=d-r \text {. }
$$

Develop a formula for $\mathrm{C}$ from

$$
C=D-R
$$




$$
C=d+\epsilon_{d}+d \epsilon_{s}+d \beta_{d}-r-\epsilon_{r}-r_{m} \epsilon_{s}-r \beta_{r}-\beta_{r t}
$$

If the bias from radiation leaking through the shadow cone is approximately $F_{s c l} R$, where $F_{s c l}$ is the fraction of the room return that is leaking through the shadow cone,

$$
C=d+\epsilon_{d}+d \epsilon_{s}+d \beta_{d}-r-\epsilon_{r}-r_{m} \epsilon_{s}-r \beta_{r}-F_{s c l} r .
$$

Assuming that the non-leakage portion of $\mathrm{R}$ is some fraction $F_{r}$ of the measured value $D$, we can simplify as

$$
C=\left(d+\epsilon_{d}+d \epsilon_{s}+d \beta_{d}\right)\left(1-F_{r}\right)-F_{s c l} r .
$$

For an ideal case, we also assume that the bias and error terms in D and $\mathrm{R}$ are small, so we establish that

$$
r=F_{r} d
$$

and simplify further as

$$
C=\left(d+\epsilon_{d}+d \epsilon_{s}+d \beta_{d}\right)\left(1-F_{r}\right)-F_{s c l} F_{r} d
$$

Splitting this into "bias" terms, error terms, and underlying terms gives

$$
C=\left(1-F_{r}\right) d+\left(1-F_{r}\right)\left(\epsilon_{d}+d \epsilon_{s}\right)+\left[\left(1-F_{r}\right) d \beta_{d}-F_{s c l} F_{r} d\right] .
$$

The underlying term is

$$
c=d\left(1-F_{r}\right)
$$

The author anticipates that betas will be small, but bias from shadow cone leakage $\left(F_{s c l} F_{r} d\right)$ could dominate the bias term:

$$
\beta_{c}=d \beta_{d}-F_{r} \beta_{d} d-F_{s c l} F_{r} d
$$

The error term is

$$
\epsilon_{c}=\epsilon_{d}+d \epsilon_{s}-F_{r} \epsilon_{d}-F_{r} d \epsilon_{s}
$$

Error terms can be represented by associated standard deviations, and they are uncorrelated,

$$
\sigma_{c}=\sqrt{\sigma_{d}^{2}+d^{2} \sigma_{s}^{2}+F_{r}^{2} \sigma_{d}^{2}+F_{r}^{2} d^{2} \sigma_{s}^{2}}
$$

Thus grouping source uncertainty with other uncertainty:

$$
\begin{gathered}
\sigma_{D}^{2}=\sigma_{d}^{2}+d^{2} \sigma_{s}^{2}, \\
\sigma_{c}=\sqrt{\sigma_{D}^{2}+F_{r}^{2} \sigma_{D}^{2}}, \text { and } \\
\sigma_{c}=\sigma_{D} \sqrt{1+F_{r}^{2}} .
\end{gathered}
$$


Without a room return shield, $F_{r}$ is greater than 1 . Detector uncertainty is probably a few percent, resulting in a much larger uncertainty from a room return shield. The effect of combining the technique with a room return shield is unclear and complicated. The shadow cone may need to be quite large to shadow the room return shield. It is not clear whether this will cause any issues. It is possible that the shadow cone could simply be a plug on the end of the room return shield. Many questions still require analysis.

\section{Shadow cone + RR shield}

If dose is the quantity of interest, then a fraction of the measured dose is from room return. If the uncertainty in room return and the principal measurement are uncorrelated, then uncertainty is proportional to

$$
\sqrt{1+F_{r}^{2}}
$$

Because the room return free dose is smaller than the principal measurement, then a scaling factor $1 /(1$ Fr) is also appropriate for uncertainty relative to the dose of interest.

$$
\frac{1}{1-F_{r}} \sqrt{1+F_{r}^{2}}
$$

where $\mathrm{F}_{\mathrm{r}}$ is the fraction of the principal measurement that is room return. If Fr is reduced to $\sim 20 \%$, then the additional uncertainty introduced by the shadow cone is not too large (if relative uncertainty is $5 \%$ when $\mathrm{Fr}=0$, it would change $6.4 \%$ when $\mathrm{F}_{\mathrm{r}}=.2$ ).

\section{A.2 ESTIMATE OF ROOM RETURN AT GODIVA IV FACILITY}

Table 4 in IER 147 [20] lists the fluences for various distances from the source. In an environment free of room return, these fluences would scale with $1 / \mathrm{r}^{2}$. However, they do not follow that law. Attempts to correct the data with a room return term that is constant throughout the room results in room returns that are about 30-60\% of the measured dose. This produces a direct transmitted dose of approximately 1/r2. This was done by adjusting the room return constant until the power fit exponent was approximately -2 , indicating $1 / \mathrm{r} 2$ behavior.

A room return that scales with $1 / \mathrm{r}$ yields room returns that are $40-53 \%$ of the measurement using the same fitting technique. The goal here is not to determine how room return scales with distance from the source, but rather to note that deviation from $1 / \mathrm{r}^{2}$ behavior in IER147 indicates that substantial room return is present. The raw data and $1 / \mathrm{r}^{2}$ fitting functions for constant room return and $1 / \mathrm{r}$ room return are shown in Figure 31. 


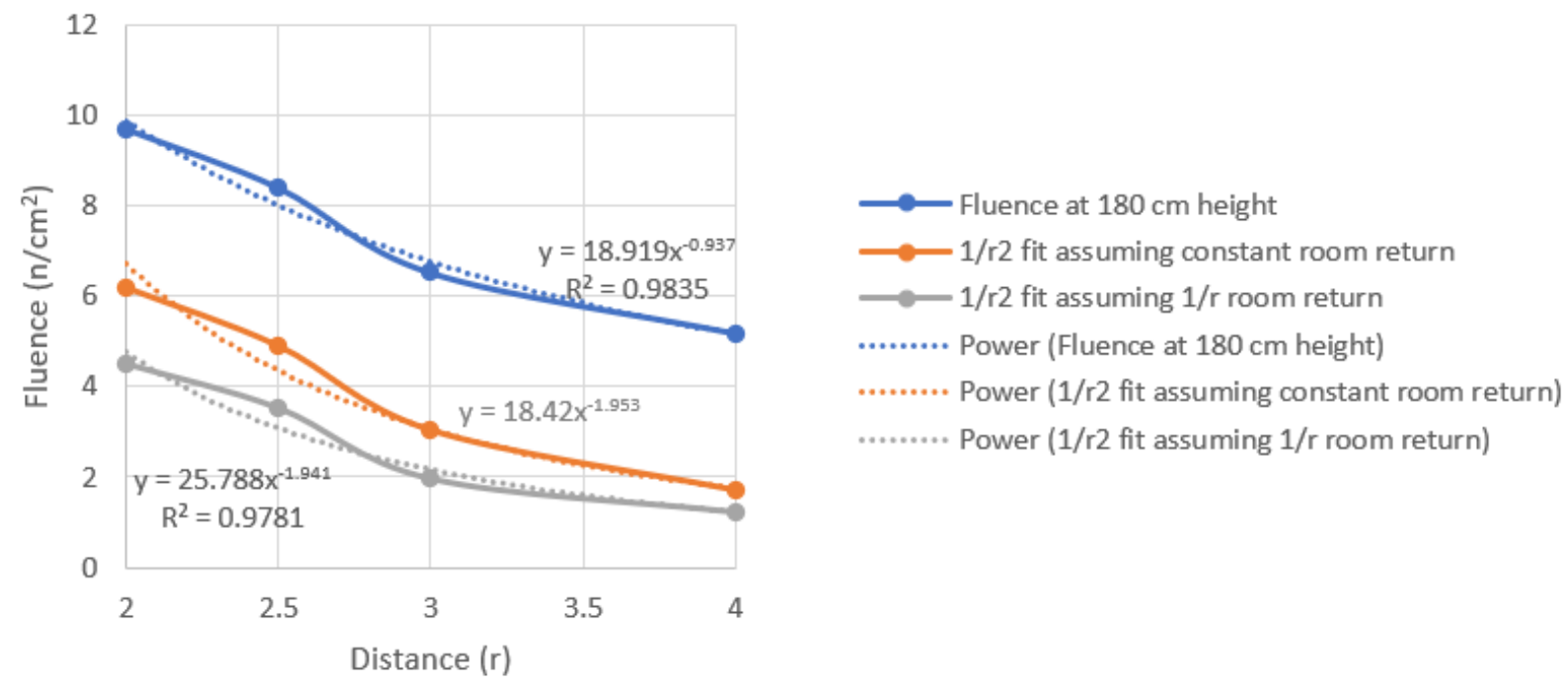

Figure 31. Raw data from Table 4 of IER 147 and $1 / \mathrm{r}^{2}$ transmitted doses for a constant room return and a $1 / \mathrm{r}$ room return.

\section{A.3 SHADOW CONE WITH GAMMA-ONLY SHIELD}

The proposed room return shield configuration described in this report is large and expensive, and it would be time consuming to fabricate. Existing practice in many shielding experiments is to use a shadow cone to address uncertainty introduced by room return. The shadow cone method has the advantages of being lighter weight, more straightforward, and simpler to fabricate. The shadow cone method has a proven track record in shielding experiments. Therefore, the shadow cone is an appealing alternative to the room return shield proposed in this report. Here, the shadow cone method is evaluated for neutrons only, using a room return shield for gammas.

This section summarizes evaluation of shadow cone performance using a gamma-only room return shield lined with CdS to take out thermal neutrons, as shown in Figure 32. This is expected to perform like a configuration using the shadow cone only for neutrons, and in its own unique way, for gammas.
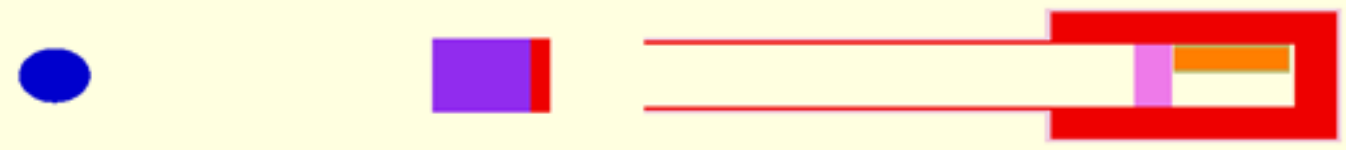

Figure 32. Lightweight gamma-only version of room return shield with CdS liner.

The shadow cone principal of operation is to absorb the radiation traveling directly from the source to the detector, so the detector only detects the room return portion of the measurement. Then the room-returnonly measurement using shadow cone configuration in Figure 33 is subtracted from a measurement with no shadow cone present Figure 34. This yields a measurement that is nominally free of room return. 


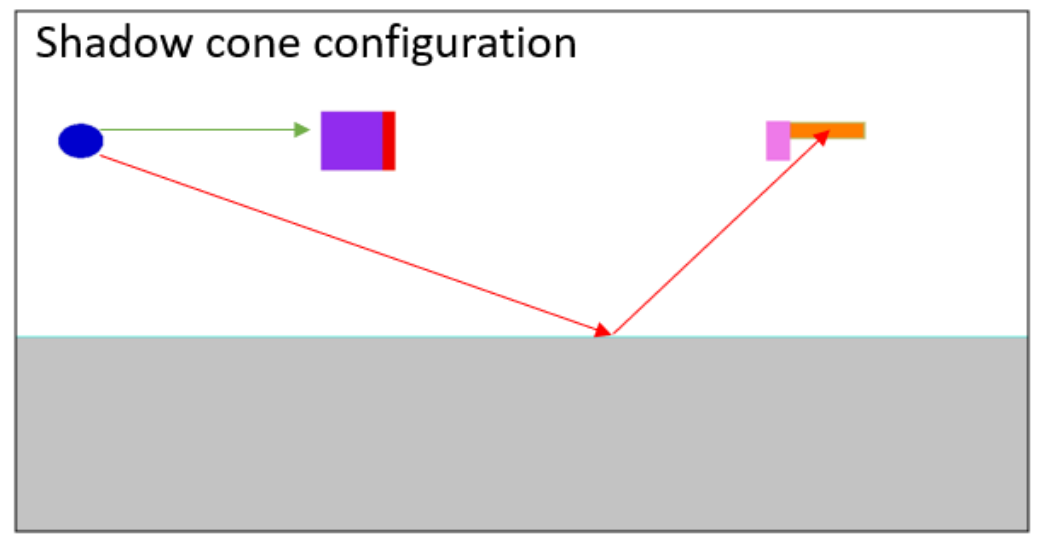

Figure 33. Configuration with shadow cone in which only room return is detected.

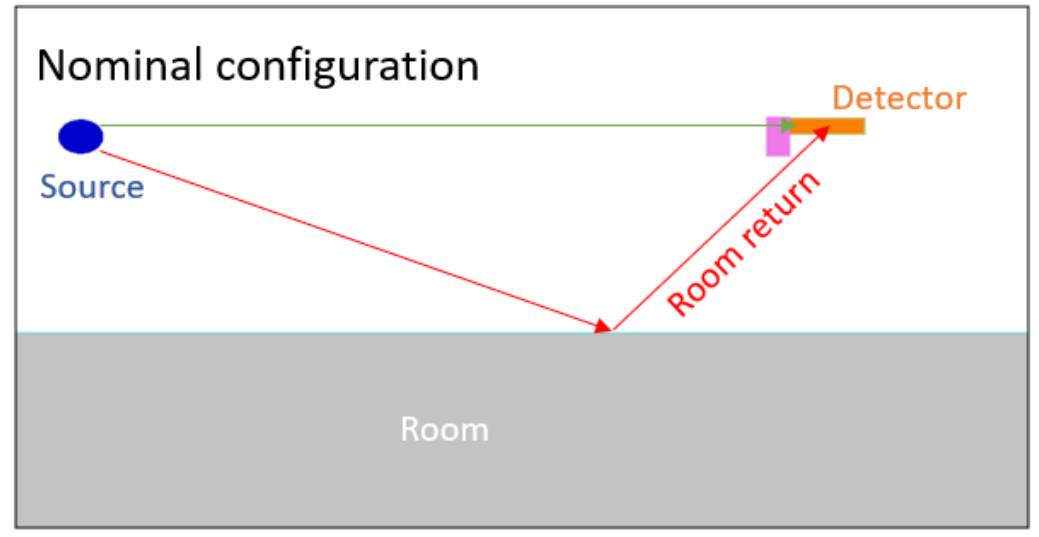

Figure 34. Configuration without shadow cone in which room return and direct transmission of radiation are detected.

The shadow cone method will be compared to the room return shield method proposed in this report, where room return is simply blocked as shown in Figure 35.

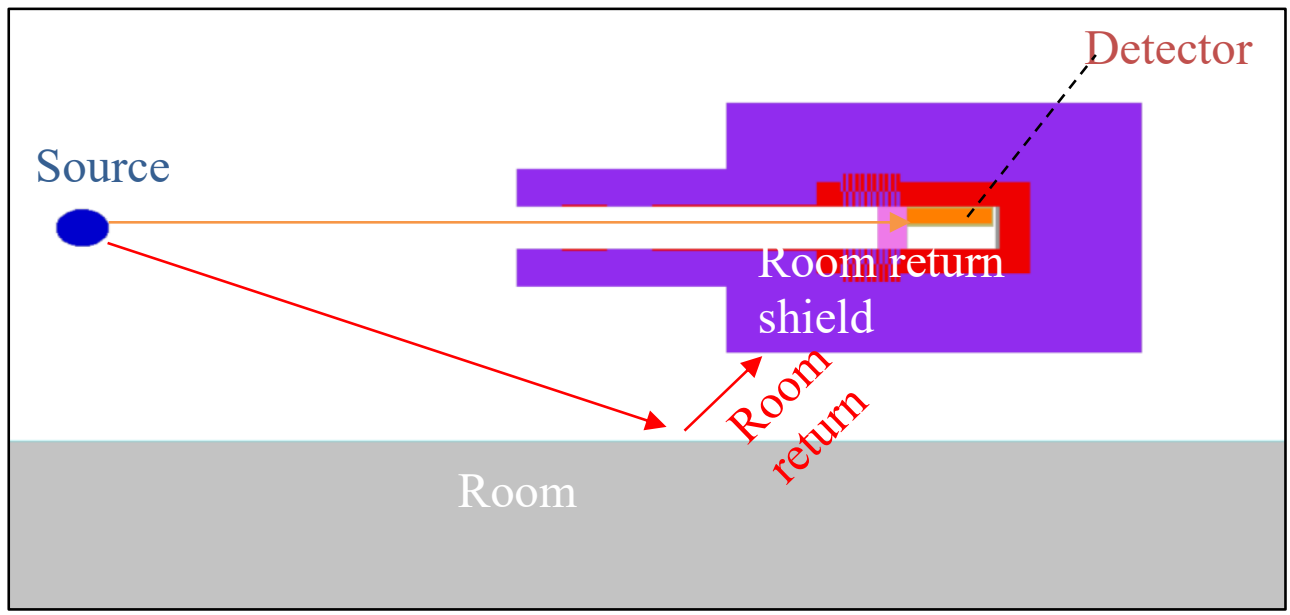

Figure 35. Configuration with room return shield to remove room return while maintaining directly transmitted radiation. 
Seven shield sample configurations were modeled using the shadow cone method and the room return shield method. Both had acceptable Monte Carlo errors, and the measurements reproduced flux values with the configuration in free space to within the Monte Carlo uncertainty. Figure 36 shows the room-tono-room ratio for the shadow cone (x axis) and the room return shield (y axis), with error bars representing the Monte Carlo error for the simulation. Note that the no-room case still retains the room return shield.

In the case of the room return, Monte Carlo errors were propagated as the Pythagorean sum of the Monte Carlo standard deviations of shadow cone measurement and the nominal configuration. Uncertainties were normalized to the room-free cases using the error propagation formula for division of a by $b$,

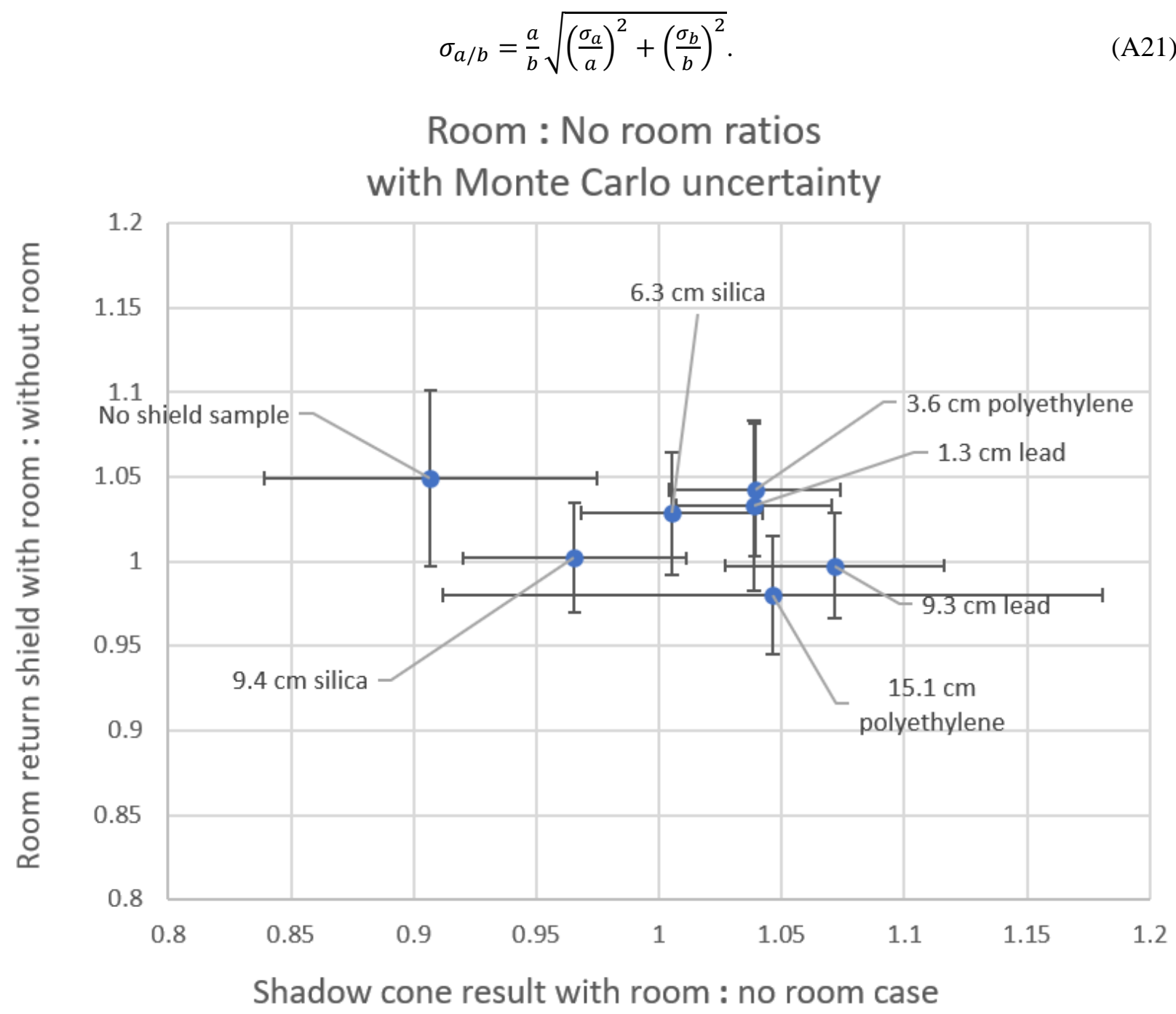

Figure 36. Comparison of room : no-room flux ratio for shadow cone (x-axis) to ratio for room return shield (y-axis). Error bars represent only the Monte Carlo uncertainty.

Figure 37 shows only the effect of measurement uncertainty. It shows the ratios of shielded measurements in a room to the values without a room. Only the uncertainty of the room measurement is included in the error bar, the no-room measurement is included mostly to normalize the quantity, so it is assigned zero uncertainty in the calculation. No Monte Carlo uncertainty is included in Figure 37. Measurements made with the room are assigned a 5\% measurement uncertainty because that is a typical 
value encountered. In Figure 37, the uncertainty in room return dominates the shadow cone measurement, with the 5\% measurement uncertainty producing an overall uncertainty of $20 \%$ when the shadow cone method is used. This issue is not observed when using the room return shield.

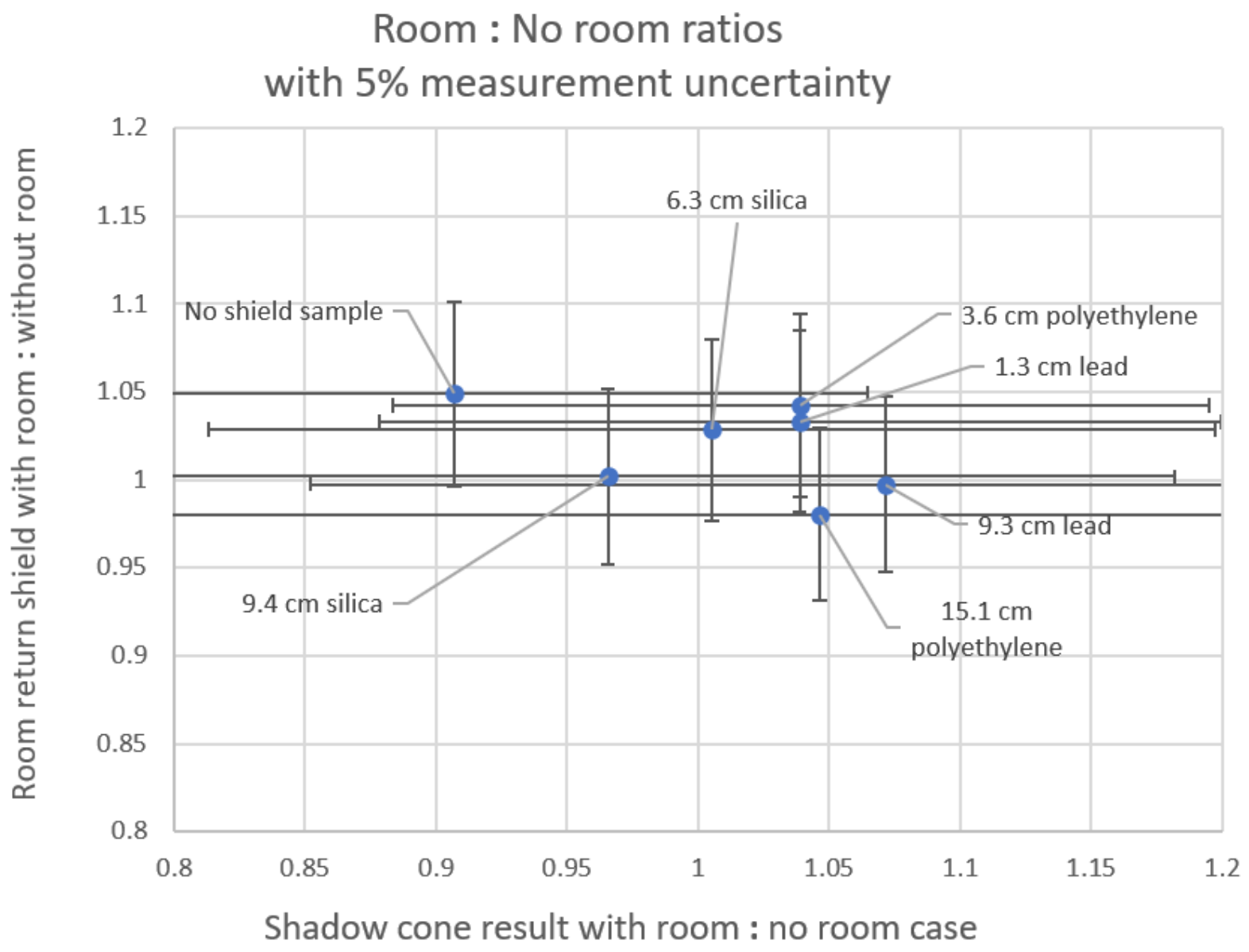

Figure 37. Comparison of room: no-room flux ratio for shadow cone (x-axis) to ratio for room return shield (y-axis). Error bars are propagated 5\% measurement uncertainties.

\section{A.4 ROOM RETURN MANAGEMENT STRATEGIES EFFECT ON SPECTRA UNCERTAINTIES AT DETECTORS}

Figure 38 presents the comparison of a silicon dioxide material of interest in a gamma-only room return shield with a CDs liner to remove thermal neutrons from room return (they cause $(n, \gamma))$. The lines are defined as follows:

- Red: measurement with no shadow cone

- Blue: measurement with shadow cone (this is basically room return)

- $\quad$ Black: room return free spectrum $=$ Red - Blue

- Yellow: alternative shadow cone measurement (no shield sample: blank control case; again, basically room return) 
- Brown: Alternate room return free spectrum $=$ Red - Yellow (this is one shadow cone measurement for many measurements performed without a shadow cone).

- Green: Same simulation with room return shield put forth in CED-1

Overall, subtraction works. To maintain the most precision, the shadow cone measurement would double the number of measurements needed. The comparison value here is the full room return shield. Note that the plot is the flux in a liquid scintillator - thus the thermalization. The error bars here are just Monte Carlo uncertainty. The shadow cone configurations (black and brown) return similar values to the room return shield result (green).
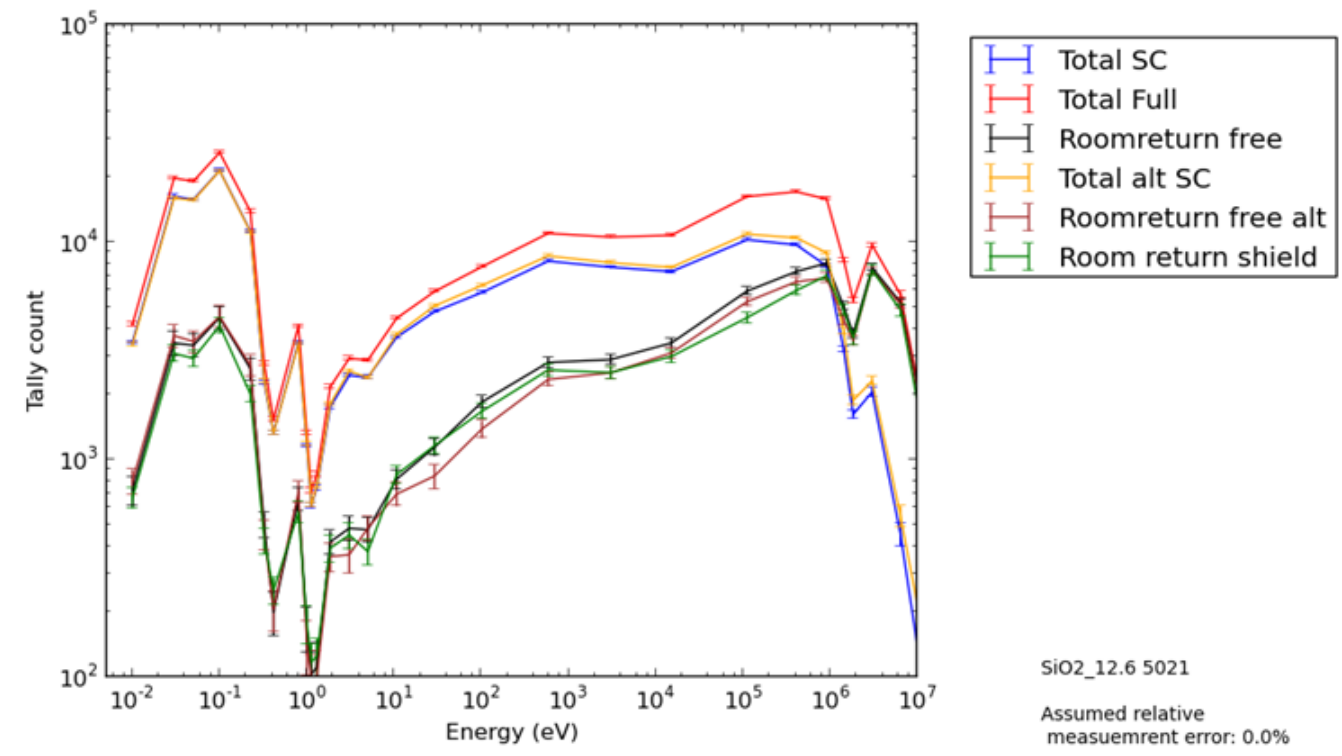

Figure 38. Neutron tallies to gauge performance of various room return management strategies for $\mathrm{S} \mathrm{SiO}_{2}$ shield sample of $6.2 \mathrm{~cm}$ with Monte Carlo error bars.

Figure 39 adds a $20 \%$ measurement uncertainty. This value is somewhat large, but it is for demonstration purposes. This uncertainty makes the error bars of the calculated shadow cone result explode (black and brown), especially where room return is a large fraction of the total signal (most of the spectrum). Note that the room return shield does not suffer from this because it blocks room return, and the shield itself is well characterized. The room return shield (green) has no subtraction, so error bars remain at $20 \%$. 

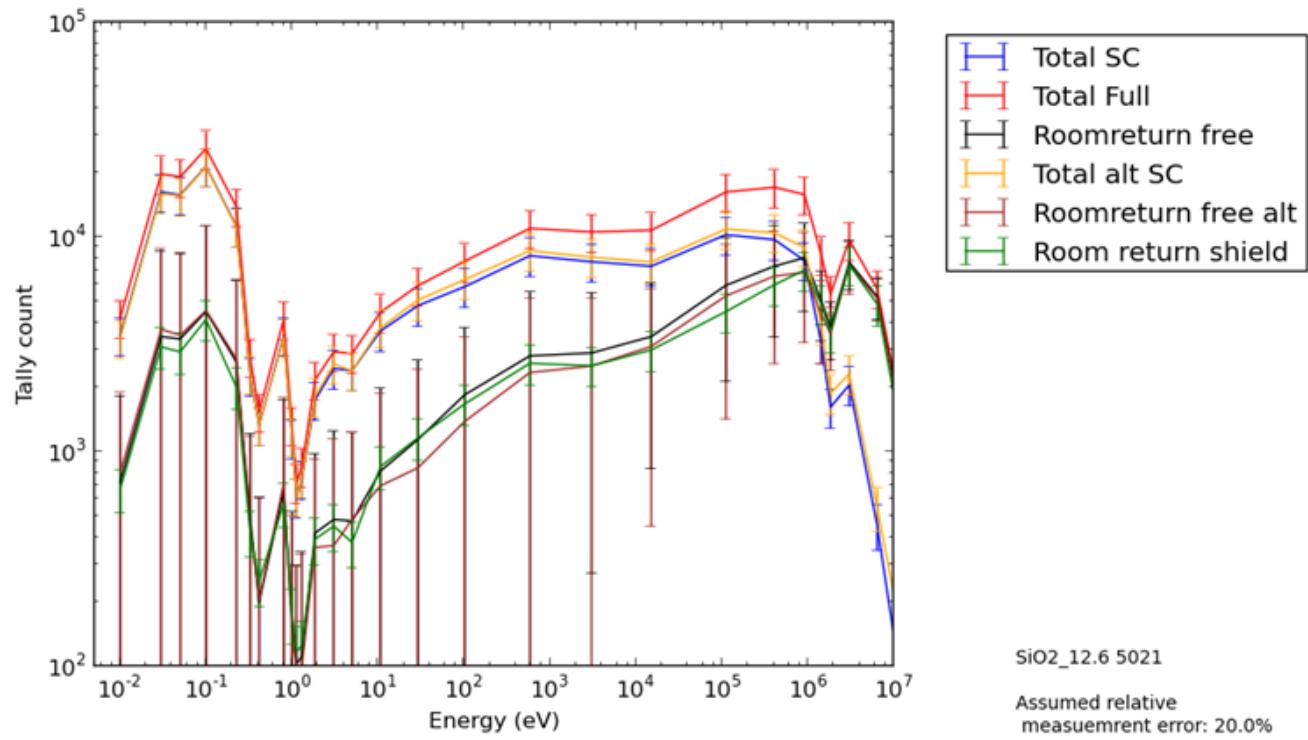

Figure 39. Neutron tallies to gauge performance of various room return management strategies for a $\mathrm{SiO}_{2}$ shield sample of $6.2 \mathrm{~cm}$ and measurement errors of $20 \%$.

Figure 40 is the gamma spectrum with Monte Carlo uncertainties for various room return management strategies. Shadow cone performance is better partly because of the gamma-only room return shield being used. This is gamma flux inside a CsI crystal. Because there is only small Monte Carlo error, subtraction works.
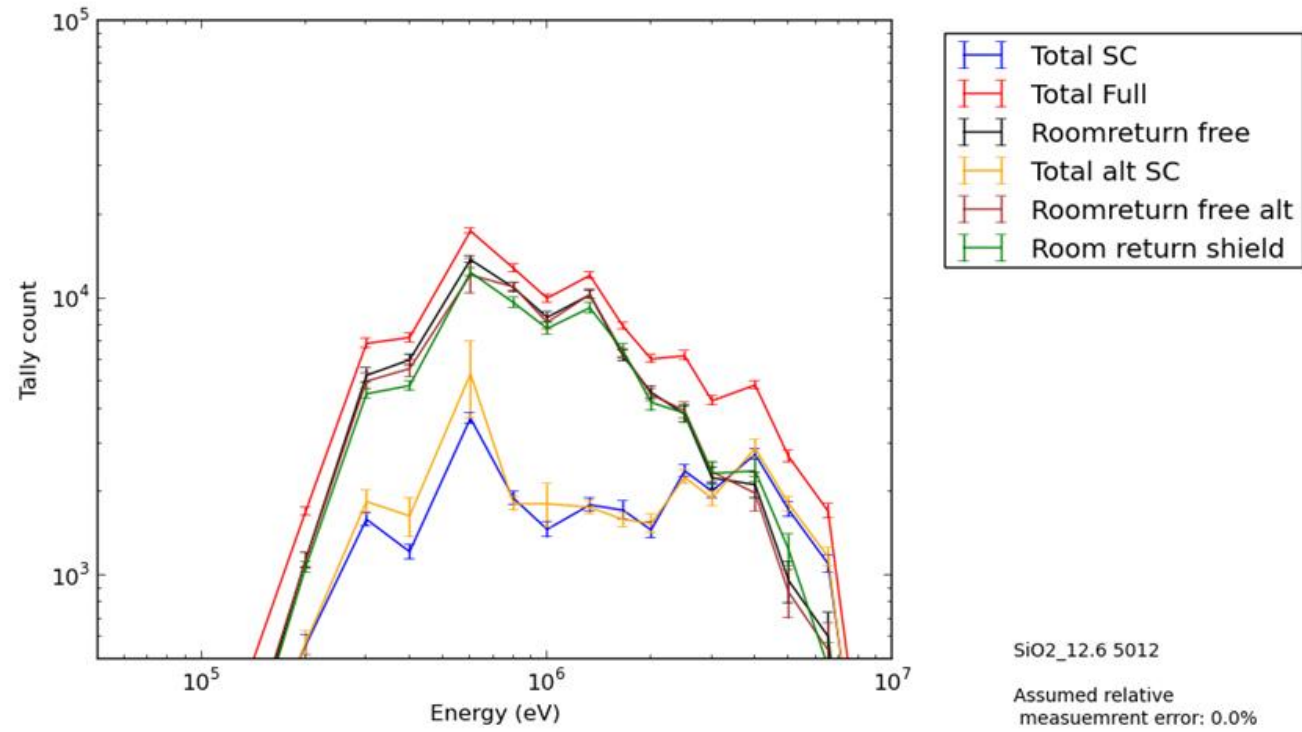

Figure 40. Gamma tallies to gauge performance of various room return management strategies for $\mathrm{S} \mathrm{SiO}_{2}$ shield sample of $6.2 \mathrm{~cm}$ and Monte Carlo error bars.

Figure 41 shows gamma spectra with $20 \%$ measurement uncertainty. Because room return is a smaller portion of the gamma dose (at least in this configuration with gamma-only room return shield + shadow cone), the addition of measurement error does not cause error to skyrocket with the use of a shadow cone 
except at the higher energies. Only the very high energy gammas have substantial room return; this could be caused by the lead itself, so the introduction of uncorrelated error through the subtraction inherent in the shadow cone method is less of a problem for the gammas under $2 \mathrm{MeV}$.
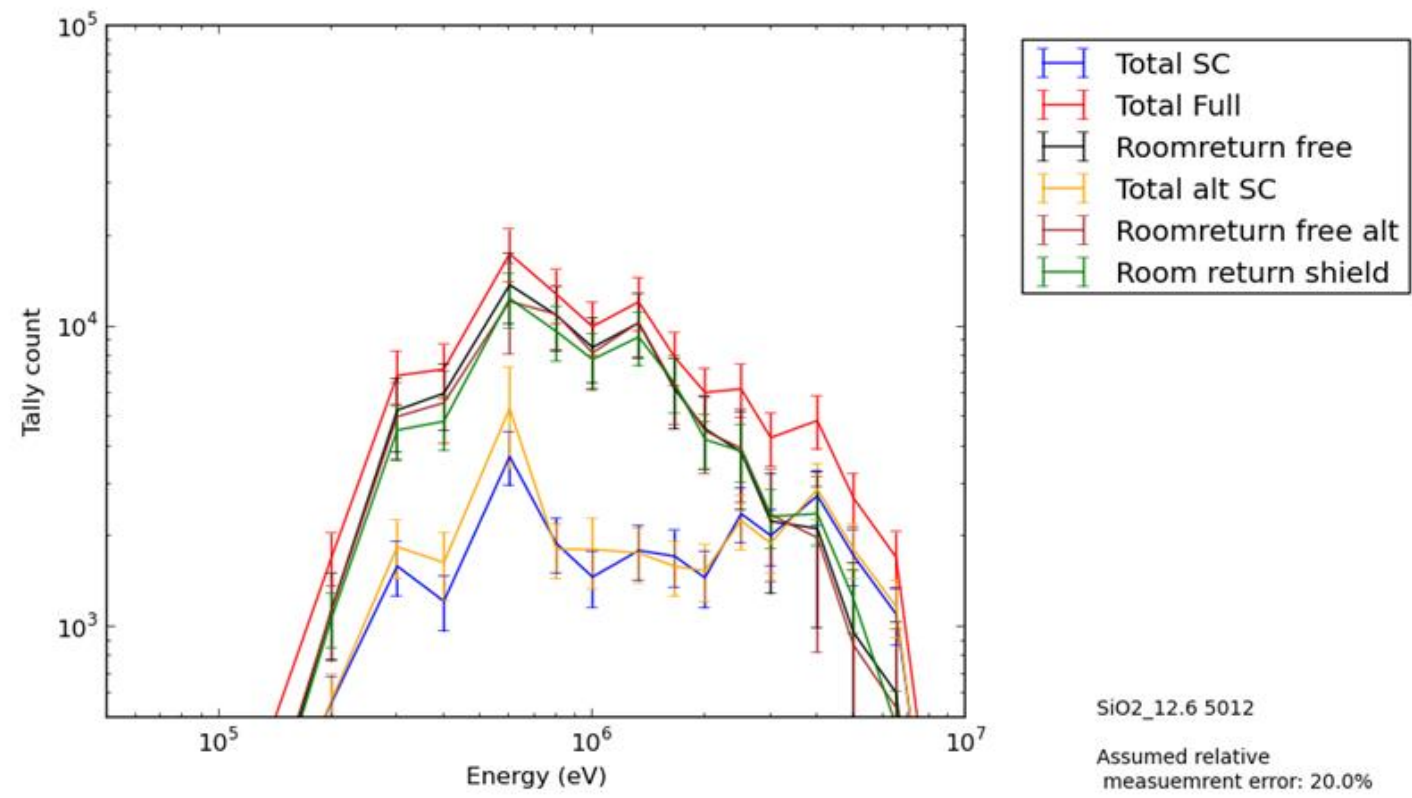

Figure 41. Gamma tallies to gauge performance of various room return management strategies for a $\mathrm{SiO}_{2}$ shield sample of $6.2 \mathrm{~cm}$ and measurement errors of $20 \%$. 


\section{APPENDIX B. PROJECT RISKS}

A list of project risks identified thus far is provided below, along with applicable mitigation strategies.

1. Funding cuts, schedule delays, cost overruns etc.

Mitigation strategy: estimate, plan, schedule, budget, monitor, adjust, repeat

2. A safety or security compliance issue occurs at NCERC which delays execution of the experiment campaign. This could take the form of:

- Delayed approval to bring an item on site

Improper procurement quality specification or acceptance test

O Other unforeseen issues, delays, etc.

3. Detector calibration/uncertainty:

Mitigation strategy: develop a clear calibration plan

4. Difficulty modeling for CED-4:

Mitigation strategy: develop MCNP model and refine MAVRIC model (support required)

5. Detector cross talk

Mitigation strategy: The experiment plan involves some evaluation of crosstalk for the electronic detectors. The passives will all be modeled completely, and they're of simple construction and small size, so crosstalk is expected to be minimal for those 


\section{APPENDIX C. EXPERIMENT REQUIREMENTS}

\section{C.1 BRINGING EQUIPMENT ONSITE}

Equipment must be screened before it is brought onsite, with a lead time of several weeks to ensure that it meets NCERC requirements in the following areas:

- Electrical safety

- Hazardous material safety (e.g., lead, detectors with flammable media)

- Security

- Heavy load safety

Some acceptance checks should be performed before or upon delivery of equipment. After acceptance checks are complete, installation can proceed. Given the lead-time for bringing equipment onsite, it is necessary to ensure that everything that might be needed is present beforehand.

\section{C.2 RECORDS}

Data acquisition system records, measurements, and equipment operation logs are necessary. Additional measurements of room temperature, assembly temperature, room return shield temperature, and room humidity may also prove useful. Additionally, photogrammetry documenting the room's layout using 3D cameras or standard photos may prove useful when developing initial benchmark models, especially to check for day-to-day changes. If appropriate, a simplified benchmark model will be developed to mimic the actual layout and can be distributed for benchmarking purposes.

\section{C.3 DISPOSITION}

Disposition of equipment will be necessary. Ideally, equipment will not have a large amount of inaccessible internal surface area. This will simplify any necessary decontamination. Hazardous materials will require special disposition.

\section{C.4 LISTING OF REQUIREMENTS}

i. $\quad$ Measurement-related requirements

a. Gammas must be measured to evaluate the adequacy of radiative capture models and data.

b. Gammas must be measured with as much spectral information as is economically possible.

c. Neutrons must be measured.

d. Neutrons must be measured with as much spectral information as is economically possible.

e. The configuration must be reconfigurable to allow for the use of a variety of shielding materials and detectors.

f. Measurements must be isolated from the room to $1 \%$ or less for several mean free paths of shielding material.

g. Measurements must not be dominated by experimental configuration (room return shielding).

h. Room return shielding must be composed of materials with well-characterized extensive (dimensional) and intensive (nuclear) properties.

i. Room return shielding must control streaming paths for radiation, so wires, other penetrations, etc., must follow curved paths.

j. Neutron room return shielding should minimize non-shielding material; non-shielding material used for structure, etc., must be evaluated for its potential to produce unmanageable secondary radiation from neutron interactions.

k. Shielding samples must be issued unique identifiers. 
1. Room return shield components should be measured and weighed to verify dimensional accuracy and the amount of shielding material present.

m. The interior of the room return shield (gamma shield and inside of neutron shield) should be assayed, or another assurance of material composition must be provided.

n. Detectors should be easy to characterize from a modeling standpoint, with known dimensions, compositions, and positions.

o. Reliable calibration of detectors is necessary.

i. The calibration source should be as similar as possible to that being measured.

ii. Calibration should be recorded adequately and must include times, as well as serial numbers of sources and detectors, and the procedure used must be specified.

p. Shielding sample source material should have samples for assay kept in case the need for assay arises in CED-4.

q. Detectors should be mounted in a fixture to avoid misplacement or variation in location of detector. A possible fixture is made from aluminum plate.

r. Room return cavity diameter and runout should be maintained within $1 \mathrm{~mm}$ or less.

ii. Safety/security

a. NCERC fire-safety requirements must be met.

b. NCERC criticality safety requirements must be met.

c. NCERC lift/heavy load safety requirements must be met.

d. Hazardous detector materials must be compatible with room return shield.

e. Lead must be powder coated in an acceptable manner to prevent/minimize spread of lead contamination.

f. Doses must remain as low as reasonably achievable (ALARA) and within NCERC requirements.

g. NCERC security requirements must be followed.

h. Sensitive information must be sufficiently protected in accordance with DOE orders and NCERC requirements.

i. Equipment in the Godiva IV area should minimize the internal surface area to reduce the decontamination burden.

j. Shielding samples and foils must be interchangeable within 30 minutes of approaching the room return shield, preferably in as short a time as practicable.

k. Detectors must be accessible within an hour of disassembly time after approaching the room return shield, preferably in as short a time as practicable.

iii. Project requirements

a. The Critical \& Subcritical Experiment Design Team $\left(\mathrm{C}_{\mathrm{E}} \mathrm{dT}\right)$ manual must be followed.

b. DOE O 414.1D Quality requirements apply.

c. The experiment must be documented in enough detail to be reproducible. 